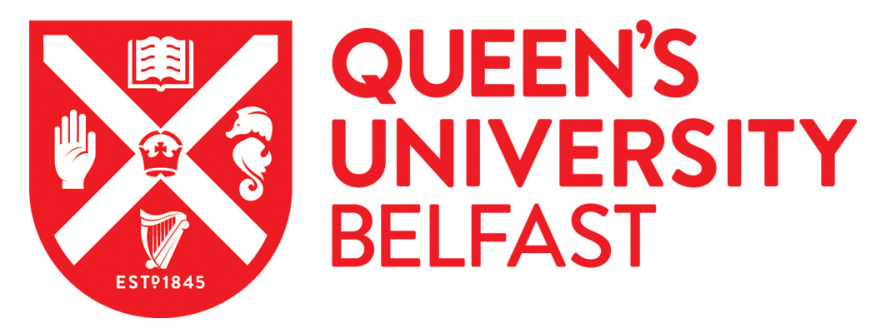

\title{
ATOMIUM: Halide molecules around the S-type AGB star W Aquilae
}

Danilovich, T., Sande, M. V. D., Plane, J. M. C., Millar, T. J., Royer, P., Amor, M. A., Hammami, K., Decock, L., Gottlieb, C. A., Decin, L., Richards, A. M. S., Beck, E. D., Baudry, A., Bolte, J., Cannon, E., Ceuster, F. D., Koter, A. D., Etoka, S., Gobrecht, D., ... Zijlstra, A. (2021). ATOMIUM: Halide molecules around the S-type AGB star W Aquilae. Astronomy and Astrophysics, 655, [A80]. https://doi.org/10.1051/0004-6361/202141757

Published in:

Astronomy and Astrophysics

Document Version:

Publisher's PDF, also known as Version of record

Queen's University Belfast - Research Portal:

Link to publication record in Queen's University Belfast Research Portal

Publisher rights

Copyright ESO 2021.

This work is made available online in accordance with the publisher's policies. Please refer to any applicable terms of use of the publisher.

\section{General rights}

Copyright for the publications made accessible via the Queen's University Belfast Research Portal is retained by the author(s) and / or other copyright owners and it is a condition of accessing these publications that users recognise and abide by the legal requirements associated with these rights.

Take down policy

The Research Portal is Queen's institutional repository that provides access to Queen's research output. Every effort has been made to ensure that content in the Research Portal does not infringe any person's rights, or applicable UK laws. If you discover content in the Research Portal that you believe breaches copyright or violates any law, please contact openaccess@qub.ac.uk. 


\title{
ATOMIUM: halide molecules around the S-type AGB star W Aquilae
}

\author{
T. Danilovich ${ }^{1, \star} \oplus$, M. Van de Sande ${ }^{1}$, J. M. C. Plane ${ }^{2}$, T. J. Millar ${ }^{3}$, P. Royer ${ }^{1}$, M. A. Amor ${ }^{4}$, \\ K. Hammami ${ }^{4}$, L. Decock ${ }^{1}$, C. A. Gottlieb ${ }^{5}$, L. Decin ${ }^{1,2}$, A. M. S. Richards ${ }^{6}$, E. De Beck ${ }^{7}$, A. Baudry ${ }^{8}$, J. Bolte ${ }^{1}$, \\ E. Cannon ${ }^{1}$, F. De Ceuster ${ }^{20,1}$, A. de Koter ${ }^{9,1}$, S. Etoka ${ }^{6}$, D. Gobrecht ${ }^{1}$, M. Gray ${ }^{6,10}$, F. Herpin ${ }^{8}$, W. Homan ${ }^{11,1}$, \\ M. Jeste ${ }^{12}$, P. Kervella ${ }^{13}$, T. Khouri ${ }^{7}$, E. Lagadec ${ }^{14}$, S. Maes ${ }^{1}$, J. Malfait ${ }^{1}$, I. McDonald ${ }^{6,22}$, K. M. Menten ${ }^{12}$, \\ M. Montargès ${ }^{13}$, H. S. P. Müller ${ }^{15}$, B. Pimpanuwat ${ }^{6,10}$, R. Sahai ${ }^{16}$, S. H. J. Wallström ${ }^{1}$, L. B. F. M. Waters ${ }^{17,18}$, \\ K. T. Wong ${ }^{19}$, J. Yates ${ }^{20}$, and A. Zijlstra ${ }^{6,21}$
}

(Affiliations can be found after the references)

Received 9 July 2021 / Accepted 9 September 2021

\begin{abstract}
Context. S-type asymptotic giant branch (AGB) stars are thought to be intermediates in the evolution of oxygen- to carbon-rich AGB stars. The chemical compositions of their circumstellar envelopes are also intermediate but have not been studied in as much detail as their carbon- and oxygen-rich counterparts. W Aql is a nearby S-type star, with well-known circumstellar parameters, making it an ideal object for in-depth study of less common molecules.

Aims. We aim to determine the abundances of $\mathrm{AlCl}$ and $\mathrm{AlF}$ from rotational lines, which have been observed for the first time towards an S-type AGB star. In combination with models based on PACS observations, we aim to update our chemical kinetics network based on these results.

Methods. We analyse ALMA observations towards W Aql of $\mathrm{AlCl}$ in the ground and first two vibrationally excited states and AlF in the ground vibrational state. Using radiative transfer models, we determine the abundances and spatial abundance distributions of $\mathrm{Al}^{35} \mathrm{Cl}, \mathrm{Al}^{37} \mathrm{Cl}$, and $\mathrm{AlF}$. We also model $\mathrm{HCl}$ and $\mathrm{HF}$ emission and compare these models to PACS spectra to constrain the abundances of these species.

Results. $\mathrm{AlCl}$ is found in clumps very close to the star, with emission confined within $0{ }^{\prime} 1$ of the star. AlF emission is more extended, with faint emission extending 0 !' 2 to $0{ }^{\prime} 6$ from the continuum peak. We find peak abundances, relative to $\mathrm{H}_{2}$, of $1.7 \times 10^{-7}$ for $\mathrm{Al}^{35} \mathrm{Cl}$, $7 \times 10^{-8}$ for $\mathrm{Al}^{37} \mathrm{Cl}$, and $1 \times 10^{-7}$ for AlF. From the PACS spectra, we find abundances of $9.7 \times 10^{-8}$ and $\leq 10^{-8}$, relative to $\mathrm{H}_{2}$, for $\mathrm{HCl}$ and $\mathrm{HF}$, respectively.

Conclusions. The AlF abundance exceeds the solar F abundance, indicating that fluorine synthesised in the AGB star has already been dredged up to the surface of the star and ejected into the circumstellar envelope. From our analysis of chemical reactions in the wind, we conclude that AlF may participate in the dust formation process, but we cannot fully explain the rapid depletion of $\mathrm{AlCl}$ seen in the wind.
\end{abstract}

Key words. stars: AGB and post-AGB - circumstellar matter - submillimeter: stars - stars: individual: W Aql stars: individual: $\chi$ Cyg

\section{Introduction}

Stars on the asymptotic giant granch (AGB) of the HertzsprungRussell diagram are an evolved form of low- and intermediatemass stars with initial masses in the range $\sim 0.8-8 M_{\odot}$. The AGB evolutionary stage is characterised by intense mass loss, of the order of $\sim 10^{-8}-10^{-4} M_{\odot} \mathrm{yr}^{-1}$ (Höfner \& Olofsson 2018). The gas ejected by these stars forms molecules and dust in an expanding region surrounding the star, known as a circumstellar envelope (CSE). The CSEs of AGB stars are rich chemical laboratories, and a large number of different molecular species have been detected towards various AGB stars (Agúndez et al. 2020).

The chemical composition of the CSE is, to the first order, determined by the photospheric abundances of $\mathrm{C}$ and $\mathrm{O}$ of the star. We define AGB stars as oxygen-rich if $\mathrm{C} / \mathrm{O}<1$ and carbon-rich if $\mathrm{C} / \mathrm{O}>1$. Generally speaking, more oxygen-bearing

\footnotetext{
$\star$ Senior Postdoctoral Fellow of the Fund for Scientific Research (FWO), Flanders, Belgium.
}

molecules are found in the CSEs of oxygen-rich stars, while carbon-bearing molecules are more prevalent in the CSEs of carbon-rich stars. There is thought to be an evolutionary progression such that all stars are oxygen-rich when they transition to the AGB and then a subset of these gradually become carbon-rich as freshly nucleosynthesised carbon is dredged up from the core of the star to the surface, increasing the $\mathrm{C} / \mathrm{O}$ ratio. S-type stars, with $\mathrm{C} / \mathrm{O} \sim 1$, are thought to be transition objects that arise during this evolutionary process (Herwig 2005). The circumstellar chemistry of S-type stars has been generally found to be intermediate between carbon- and oxygen-rich chemistry (see, for example, Danilovich et al. 2014).

Halogen-bearing molecules have not been extensively studied in the CSEs of many AGB stars. Chlorine has been found to be a tracer of metallicity (Maas et al. 2016), and fluorine is thought to be produced in AGB stars and dredged up to the surface of the star (Kobayashi et al. 2020). By understanding the total abundance of $\mathrm{Cl}$ or $\mathrm{F}$ around AGB stars, we can better 
understand their metallicities or AGB ages, respectively. To date, halide molecules have been studied in most detail towards the nearby, high mass-loss rate carbon star CW Leo (IRC+10216), towards which $\mathrm{AlCl}, \mathrm{NaCl}, \mathrm{KCl}, \mathrm{AlF}, \mathrm{HCl}$, and $\mathrm{HF}$ have been detected (Cernicharo \& Guelin 1987; Cernicharo et al. 2010; Agúndez et al. 2011, 2012). Aside from CW Leo, halogenbearing molecules have only been detected towards a handful of AGB stars, such as $\mathrm{Al}^{35} \mathrm{Cl}$ tentatively seen towards the oxygenrich stars IK Tau and R Dor (Decin et al. 2017), and $\mathrm{NaCl}$ seen towards IK Tau (Milam et al. 2007; Decin et al. 2018) and tentatively R Dor (De Beck \& Olofsson 2018). No spectrally resolved halogen-bearing species have been previously reported towards any S-type stars ${ }^{1}$, although spectrally unresolved infrared observations of $\mathrm{HCl}$ in the atmosphere of the S-type star R And have been reported by Yamamura et al. (2000).

Agúndez et al. (2020) undertook an extensive study of molecular abundances in the inner regions of AGB CSEs under the assumption of thermochemical equilibrium, making several predictions for molecular abundances in the inner 10 stellar radii $\left(R_{\star}\right)$. They predict $\mathrm{AlF}$ and $\mathrm{AlCl}$ to be the dominant $\mathrm{F}$ - and $\mathrm{Cl}$ bearing molecules from $\sim 3 R_{\star}$ outwards for S-type stars, with $\mathrm{HF}$ and $\mathrm{HCl}$ dominating the innermost regions $\left(\lesssim 3 R_{\star}\right)$. Hence, we should expect to see both aluminium and hydrogen halides towards S-type stars.

In recent years, W Aql has become the most-studied S-type AGB star, thanks to a combination of its proximity $(374 \pm 22 \mathrm{pc}$, Gaia Collaboration 2016, 2021; Lindegren et al. 2021), moderately high mass-loss rate $\left(3 \times 10^{-6} M_{\odot} \mathrm{yr}^{-1}\right.$, Ramstedt et al. 2017 ), and equatorial position in the sky (declination $\sim-7^{\circ}$ ). It has been observed by two instruments aboard Herschel ${ }^{2}$ (Mayer et al. 2013; Danilovich et al. 2014) and the Atacama Large Millimetre/sub-millimetre Array (ALMA, Ramstedt et al. 2017; Brunner et al. 2018), as well as a variety of other telescopes (see for example De Beck \& Olofsson 2020), which helped constrain the conditions in its CSE. In addition to (sub)millimetre observations, optical and infrared observations have provided information on the dust around this star (Hony et al. 2009; Mayer et al. 2013; Ramstedt et al. 2011) and its companion, which has been characterised as an F9 main sequence star (Danilovich et al. 2015a) located at a projected distance of 0.'46 (Ramstedt et al. 2011), which corresponds to approximately $170 \mathrm{AU}$ at the distance given by Gaia.

In this study we focus on the rotational lines of halogenbearing molecules towards $\mathrm{W}$ Aql, especially $\mathrm{AlCl}$ and $\mathrm{AlF}$, which were observed with ALMA. These data are presented in Sect. 2. Additionally, we examine observations of $\mathrm{HCl}$ and $\mathrm{HF}$ obtained by Herschel/PACS (Sect. 3). Radiative transfer modelling is performed for all four of these molecules (Sect. 4), with the results presented in Sect. 5. We discuss our results in the context of the literature and use them to update our chemical kinetics model in Sect. 6. Section 7 summarises our conclusions.

\section{ALMA observations of $\mathrm{AICl}$ and AIF}

As part of the ATOMIUM ${ }^{3}$ programme (2018.1.00659.L, PI: L. Decin), W Aql was observed with three configurations

\footnotetext{
1 Aside from a misidentification of $\mathrm{NaCl}$ towards $\mathrm{W}$ Aql by De Beck \& Olofsson (2020), which will be discussed further in Sect. 3.1.

2 Herschel is an ESA space observatory with science instruments provided by European-led Principal Investigator consortia and with important participation from NASA.

3 https://fys.kuleuven.be/ster/research-projects/ aerosol/atomium/atomium
}

of ALMA, which we refer to as the compact (angular resolution 1.' $11 \times 0$. .'88 at $262.2 \mathrm{GHz}$ and maximum recoverable scale $($ MRS $)=8 . \prime 9)$, mid (angular resolution $0.374 \times 0.250$ and MRS =3".9), and extended (angular resolution 0.'024 ×0.'021 and MRS =0.'4) arrays (see Decin et al. 2020; Gottlieb et al. 2021 , for details). These three datasets were combined to produce more sensitive data cubes to allow us to examine the observations in more detail. When data from the different ALMA configurations are combined, different resolutions can be chosen to emphasise different aspects of the data. We found the most useful combined data to have angular resolution of $32 \times 30$ mas for $\mathrm{AlCl}$ and $150 \times 130$ mas for AlF. For this study, we aim to use the best dataset in each context, as will be discussed in detail below.

We detected $\mathrm{Al}^{35} \mathrm{Cl}$ in the ground, first, and second excited vibrational states $(v=0,1,2), \mathrm{Al}^{37} \mathrm{Cl}$ in the ground and first excited vibrational states $(v=0,1)$, and AlF in the ground vibrational state $(v=0)$. We also use tentative or undetected lines of $\mathrm{Al}^{35} \mathrm{Cl}$ in the third vibrationally excited state, $\mathrm{Al}^{37} \mathrm{Cl}$ in the second vibrationally excited state, and AlF in the first vibrationally excited state as upper limits when we perform our radiative transfer analysis of the data (see Sects. 4 and 5). These lines are listed with their frequencies, upper level energies and central velocities in Table 1. The central velocities were found by fitting Gaussian profiles to spectra extracted from the combined cubes. Based on these central velocities, we find an average LSR velocity of $-23.1 \pm 0.9 \mathrm{~km} \mathrm{~s}^{-1}$, which is in good agreement with the values found by Danilovich et al. (2014) and De Beck \& Olofsson (2020) from single dish observations of a variety of molecules.

Angular sizes are given in Table 1 for sufficiently bright lines. They have been measured by: examining zeroth moment maps (of the velocity-integrated emission) of each line over the velocity range indicated in Table 1; and creating contours enclosing the flux at the $2 \sigma$ level. The $2 \sigma$ level was chosen since this gives a more accurate estimate of total extent, including weaker emission, than the $3 \sigma$ level. Isolated islands only detected at $2 \sigma$ levels are not included since we require at least $3 \sigma$ certainty to consider emission to be detected. This gave a table of $x$ and $y$ values of the coordinates enclosing the flux, centred on the star, which could be transformed to polar coordinates $r, \theta$. To reduce random noise affecting the contour, we binned and averaged the values with at least 10 samples per bin, corresponding to angular ranges of $\geq 60^{\circ}$. We then measured the longest $\left(R_{\max }\right)$ and shortest $\left(R_{\min }\right)$ radial distances from the continuum peak to the binned contour. To give an indication of the regularity or irregularity of the shape of the emission, we also note the angle $\left(\theta_{\mathrm{R}}\right)$ between the radii of the nearest and farthest angle of the contour. If these are orthogonal the radii can correspond to semi-major and semi-minor axes, suggesting a more regular distribution, but if the angle is very different from $90^{\circ}$, then the distribution is asymmetric. For AlF the measurement was done for data that had been combined with a taper of 0.2 , giving a lower resolution image but avoiding the irregularities seen in Fig. 3. The uncertainty in the radii is $\sim 3$ mas for $\mathrm{AlCl}$ and $\sim 18$ mas for $\mathrm{AlF}$.

We characterise the 1D spectra extracted from ALMA cubes by an aperture size, which is the size of a circular region over which the spectrum has been extracted, and is always centred on the continuum peak. Since the $\operatorname{AlF}(7 \rightarrow 6) v=0$ line is relatively weak, we average channels to give a lower velocity resolution of $\sim 2.5 \mathrm{~km} \mathrm{~s}^{-1}$ (compared with $\sim 1.1 \mathrm{~km} \mathrm{~s}^{-1}$ for $\mathrm{AlCl}$ ) and a higher signal-to-noise ratio. For our modelling, we also use what we refer to as azimuthally averaged radial profiles of the ALMA lines. These are extracted from the zeroth moment maps by obtaining the average flux in concentric annuli, plus 
T. Danilovich et al.: ATOMIUM: Halide molecules around the S-type AGB star W Aquilae

Table 1. Properties of lines of $\mathrm{AlCl}$ and $\mathrm{AlF}$ covered by ATOMIUM towards W Aql.

\begin{tabular}{|c|c|c|c|c|c|c|c|c|c|c|c|}
\hline Molecule & $v^{\mathrm{T}}$ & $\begin{array}{l}\text { ansition } \\
\qquad J^{\prime} \rightarrow J\end{array}$ & $\begin{array}{l}\text { Frequency } \\
\quad(\mathrm{GHz})\end{array}$ & $\begin{array}{l}E_{\text {up }} \\
(\mathrm{K})\end{array}$ & $\begin{array}{l}\text { Aperture } \\
\text { (mas) }\end{array}$ & $\begin{array}{c}v_{\text {cent }} \\
\left(\mathrm{km} \mathrm{s}^{-1}\right)\end{array}$ & $\begin{array}{l}\text { Int. flux } \\
\left(\mathrm{Jy} \mathrm{km} \mathrm{s}^{-1}\right)\end{array}$ & $\begin{array}{l}R_{\max } \\
\text { (mas) }\end{array}$ & $\begin{array}{l}R_{\min } \\
\text { (mas) }\end{array}$ & $\begin{array}{l}\theta_{\mathrm{R}} \\
\left(^{\circ}\right)\end{array}$ & $\begin{array}{l}\text { Vel. range } \\
\left(\mathrm{km} \mathrm{s}^{-1}\right)\end{array}$ \\
\hline $\mathrm{Al}^{35} \mathrm{Cl}$ & 0 & $18 \rightarrow 17$ & 262.219 & 120 & $\begin{array}{c}200 \\
80\end{array}$ & $\begin{array}{l}-23.8 \\
-23.7\end{array}$ & $\begin{array}{l}0.415 \\
0.279\end{array}$ & $\begin{array}{l}93 \\
\ldots\end{array}$ & $\begin{array}{l}39 \\
\ldots\end{array}$ & $\begin{array}{c}100 \\
\ldots\end{array}$ & $\begin{array}{c}-29.4,-16.0 \\
\ldots\end{array}$ \\
\hline $\mathrm{Al}^{35} \mathrm{Cl}$ & 1 & $17 \rightarrow 16$ & 246.037 & 794 & 80 & -23.2 & 0.111 & 62 & 28 & 120 & $-14.5,-31.2$ \\
\hline $\mathrm{Al}^{35} \mathrm{Cl}$ & 1 & $18 \rightarrow 17$ & 260.491 & 807 & 80 & -22.7 & $0.138^{(\dagger)}$ & $\ldots$ & $\ldots$ & $\ldots$ & $\ldots$ \\
\hline $\mathrm{Al}^{35} \mathrm{Cl}$ & 2 & $16 \rightarrow 15$ & 230.053 & 1463 & 80 & -22.3 & 0.066 & $\ldots$ & $\ldots$ & $\ldots$ & $\ldots$ \\
\hline $\mathrm{Al}^{35} \mathrm{Cl}$ & 2 & $17 \rightarrow 16$ & $244.414^{(*)}$ & 1475 & 80 & -23.2 & 0.048 & $\ldots$ & $\ldots$ & $\ldots$ & $\ldots$ \\
\hline $\mathrm{Al}^{35} \mathrm{Cl}$ & 2 & $18 \rightarrow 17$ & 258.773 & 1488 & 80 & -22.7 & 0.068 & $\ldots$ & $\ldots$ & $\ldots$ & $\ldots$ \\
\hline $\mathrm{Al}^{35} \mathrm{Cl}$ & 3 & $15 \rightarrow 14$ & 214.265 & 2117 & 80 & $\ldots$ & ND & $\ldots$ & $\ldots$ & $\ldots$ & $\ldots$ \\
\hline $\mathrm{Al}^{35} \mathrm{Cl}$ & 3 & $16 \rightarrow 15$ & 228.534 & 2128 & 80 & $\ldots$ & ND & $\ldots$ & $\ldots$ & $\ldots$ & $\ldots$ \\
\hline $\mathrm{Al}^{37} \mathrm{Cl}$ & 0 & $16 \rightarrow 15$ & 227.643 & 93 & 200 & -23.3 & 0.209 & 63 & 23 & 200 & $-27.8,-18.7$ \\
\hline $\mathrm{Al}^{37} \mathrm{Cl}$ & 1 & $18 \rightarrow 17$ & 254.396 & 796 & 80 & -23.7 & 0.087 & $\ldots$ & $\ldots$ & $\ldots$ & $\ldots$ \\
\hline $\mathrm{Al}^{37} \mathrm{Cl}$ & 1 & $19 \rightarrow 18$ & $268.509^{(*)}$ & 809 & 80 & -23.5 & 0.084 & $\ldots$ & $\ldots$ & $\ldots$ & $\ldots$ \\
\hline $\mathrm{Al}^{37} \mathrm{Cl}$ & 2 & $18 \rightarrow 17$ & $252.738^{(*)}$ & 1020 & 80 & -20.8 & 0.083 & $\ldots$ & $\ldots$ & $\ldots$ & $\ldots$ \\
\hline $\mathrm{AlF}$ & 0 & $7 \rightarrow 6$ & 230.794 & 44 & 600 & -24.3 & 0.635 & 769 & 284 & 91 & $-50.7,5.2$ \\
\hline & & & & & 300 & -23.8 & 0.383 & $\ldots$ & $\cdots$ & $\ldots$ & $\ldots$ \\
\hline $\mathrm{AlF}$ & 1 & $7 \rightarrow 6$ & 228.717 & 1185 & 200 & $\ldots$ & ND & $\ldots$ & $\ldots$ & $\ldots$ & $\ldots$ \\
\hline
\end{tabular}

Notes. ${ }^{(a)}$ Integrated flux density. ${ }^{(*)}$ indicates a tentative detection. ${ }^{(\dagger)}$ Indicates an uncertain measurement due to a partial overlap with the wing of an adjacent line. Aperture gives the radius of the spectral extraction aperture; $R_{\min }$ and $R_{\max }$ give the minimum and maximum angular extents of the emission, measured from the continuum peak for a zeroth moment map made by summing over the channels in the velocity range indicated, and $\theta_{\mathrm{R}}$ is the angle between $R_{\min }$ and $R_{\max }$. Angular extents are omitted for unresolved emission. ND indicates a non-detection.

References. Line data retrieved from CDMS (Müller et al. 2001, 2005) with $\mathrm{Al}^{35} \mathrm{Cl}$ and $\mathrm{Al}{ }^{37} \mathrm{Cl}$ frequencies from Wyse \& Gordy (1972) and Hensel et al. (1993) and AlF frequencies from Wyse et al. (1970) and Hoeft et al. (1970).
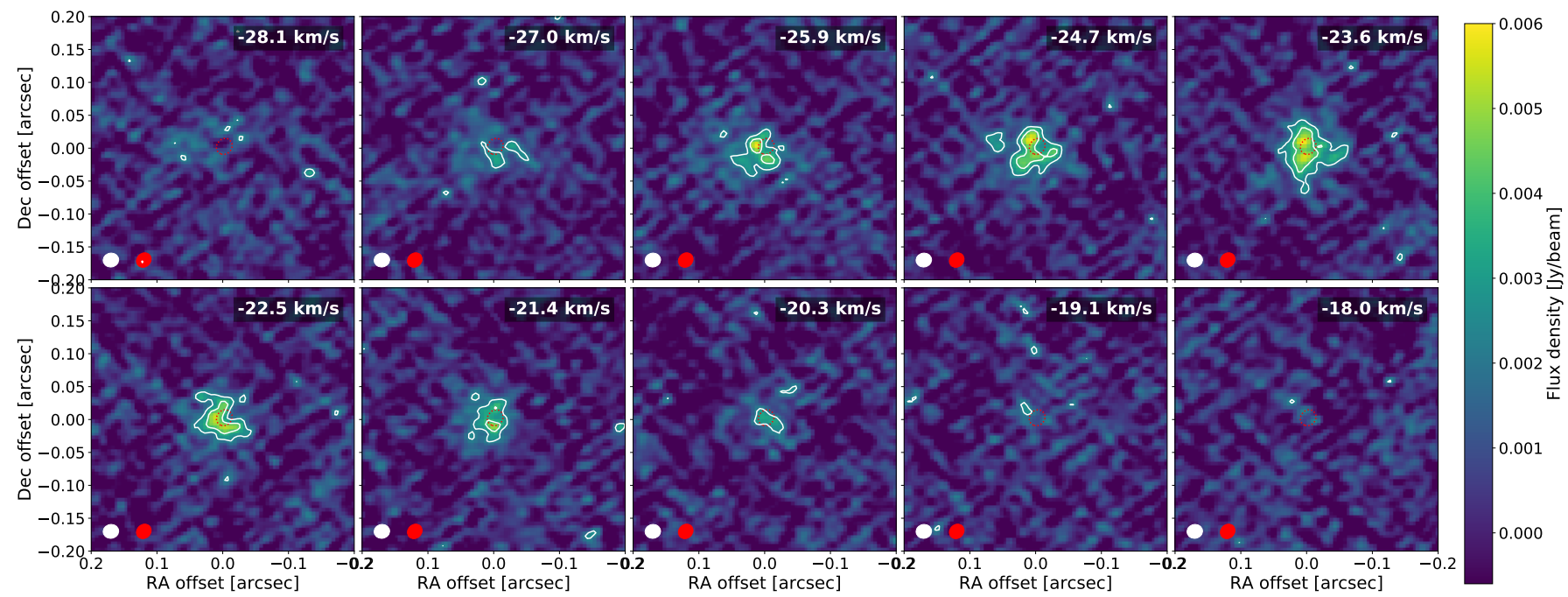

Fig. 1. Channel maps of $\mathrm{Al}^{35} \mathrm{Cl}(18 \rightarrow 17)$ observed towards W Aql using ALMA in the extended array configuration. The dotted red contours enclose $50 \%$ of the total continuum flux. The white contours indicate levels of 3 and $5 \sigma$. The ellipses in the bottom left of each panel indicate the synthetic beams for the molecular emission (white) and continuum emission (red).

the flux in the central circular region, centred on the continuum peak. These radial profiles allow us to more easily compare ALMA data with our spherically symmetric models (see Sect. 4 for details of modelling).

\section{1. $\mathrm{AlCl}$}

The $\mathrm{AlCl}$ lines seen towards W Aql are all fairly compact, including the $v=0$ lines. The channel maps for $\mathrm{Al}^{35} \mathrm{Cl}(18 \rightarrow 17)$ in the ground vibrational state, observed with the ALMA extended array, are shown in Fig. 1. There it can be seen that the emission is resolved and clumpy, but seen within $0{ }^{\prime} 1$ of the star. The $\mathrm{Al}^{35} \mathrm{Cl} v=1$ lines and the $\mathrm{Al}^{37} \mathrm{Cl}(16 \rightarrow 15)$ line in the ground vibrational state are found in similar regions but are seen less clearly, due to their expected lower intensities. We show the zeroth moment maps of $\mathrm{Al}^{35} \mathrm{Cl}(18 \rightarrow 17)$ and $\mathrm{Al}^{37} \mathrm{Cl}(16 \rightarrow 15)$ in the ground vibrational state, and $(17 \rightarrow 16)$ in the first vibrationally excited state in Fig. 2. The zeroth moment maps were all constructed so as to include all of the line flux with no contamination from adjacent lines and with minimal dilution due to line-free noise-dominated channels. The zeroth moment map of $\mathrm{Al}^{35} \mathrm{Cl} v=0(18 \rightarrow 17)$ shows the emission is not centred on the 

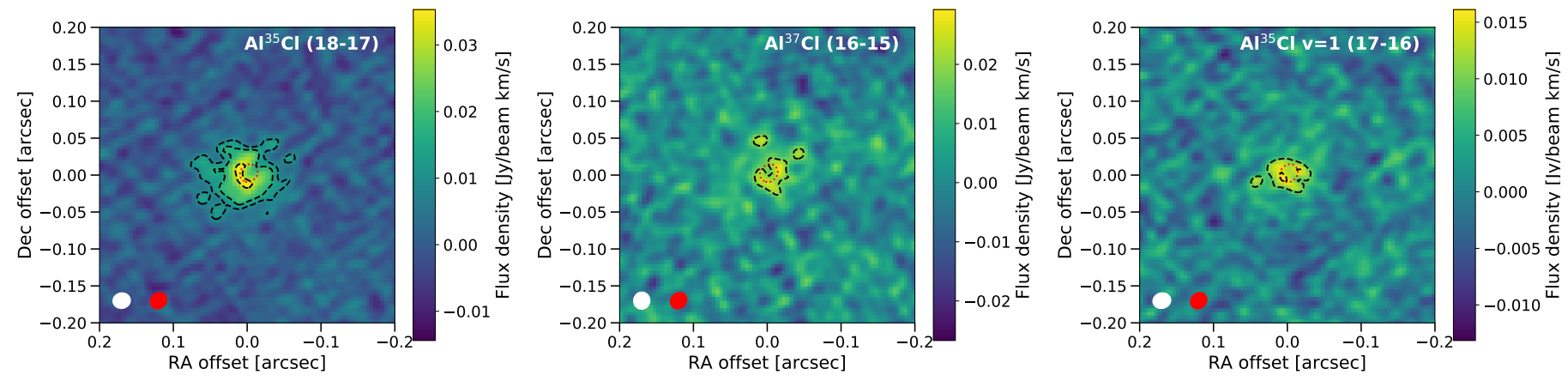

Fig. 2. Zeroth moment maps of AlCl observed towards W Aql using ALMA in the extended array configuration. Transitions are labelled in the top right of each panel. The black contours indicate levels of 3,5, and $10 \sigma$. The dotted red contour encloses $50 \%$ the total continuum flux. The ellipses in the bottom left of each panel indicate the synthetic beams for the molecular emission (white) and continuum emission (red).

star, with more emission seen to the south-east than the northwest. However, the emission from the other transitions shown in Fig. 2 is not consistently offset in the same direction. We conclude that the cause of this asymmetry is either noise or clumpy emission, rather than a specific directional bias for the formation of $\mathrm{AlCl}$, though such bias is not decisively ruled out. The spectral lines of $\mathrm{AlCl}$ are presented in conjunction with our models in Sect. 5, where we show all the lines extracted for an aperture of radius $0{ }^{\prime} 08$ and include the $\mathrm{Al}^{35} \mathrm{Cl} v=0(18 \rightarrow 17)$ line extracted for a radius of 0.2 , to ensure all the flux is captured for the purposes of comparisons with our model.

\subsection{AlF}

The ground state AlF emission is more extended than $\mathrm{AlCl}$. It has a bright central region, within $\sim 0$.' 15 of the star, and a more extended region of faint, clumpy emission. The extended emission is seen most clearly in a zeroth moment map with a lower angular resolution of $150 \times 130$ mas (Fig. 3 ), which allows us to see the faint emission above the noise, with dashed white contours outlining the emission down to the $3 \sigma$ level. As can be seen in Fig. 3, the extended diffuse emission is mostly found to the north of the stellar position. Relative to the stellar continuum peak, the diffuse emission extends to $\sim 0$.' $^{\prime} 5$ north, $\sim 00^{\prime} 6$ east, $\sim 0^{\prime} .4$ west, and only $\sim 0^{\prime} .2$ south. We include contours of the $\mathrm{Al}^{35} \mathrm{Cl}(18 \rightarrow 17)$ zeroth moment map (with an angular resolution of $32 \times 30$ mas) in Fig. 3. Although the offsets noted for $\mathrm{AlF}$ and $\mathrm{Al}^{35} \mathrm{Cl}$ are not in the same direction, we find that the central peak of AlF emission corresponds reasonably well with the region of $\mathrm{Al}^{35} \mathrm{Cl}$ emission.

\section{Observations of other halide molecules}

\section{1. $\mathrm{NaCl}$ and $\mathrm{KCl}$}

Several transitions of $\mathrm{NaCl}$ and $\mathrm{KCl}$ were covered in the ATOMIUM observations but not detected towards W Aql. ATOMIUM detections of these two molecules were made towards the higher mass-loss rate oxygen-rich stars (and the red supergiant VX Sgr) and will be examined in more detail in a future work. We calculate the rms values as detection limits for $\mathrm{NaCl}$ and $\mathrm{KCl}$ towards W Aql in Appendix A.1.

In their recent study, De Beck \& Olofsson (2020) analysed a spectral survey of W Aql conducted by the Atacama Pathfinder Experiment (APEX). They find emission from 13 species and their isotopologues, in addition to two unidentified lines (the latter falling outside of our observed frequency range). They

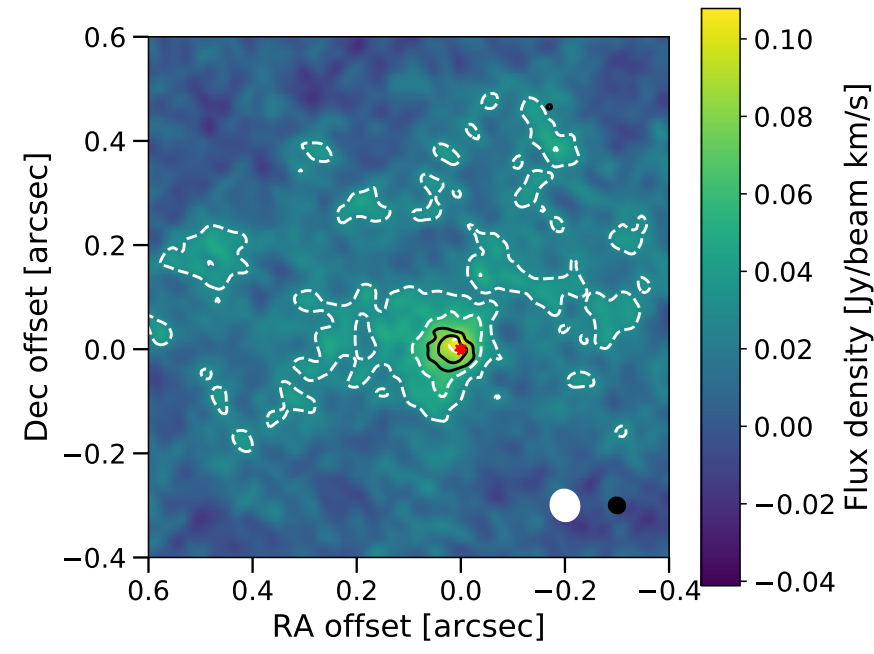

Fig. 3. Zeroth moment map of the $\operatorname{AlF}(7 \rightarrow 6)$ line in the ground vibrational state (colours and white dashed contours) plotted with the zeroth moment map of the $\mathrm{Al}^{35} \mathrm{Cl}(18 \rightarrow 17)$ line in the ground vibrational state (black solid contours). The contours are plotted at levels of 3, 5, and $10 \sigma$ for $\mathrm{AlF}$ and 5 and $10 \sigma$ for $\mathrm{Al}^{35} \mathrm{Cl}$. The red star indicates the position of the continuum peak. The white and black ellipses in the bottom right indicate the synthetic beam sizes for the $\mathrm{AlF}$ and $\mathrm{Al}^{35} \mathrm{Cl}$ emission, respectively.

do not detect $\mathrm{AlCl}$ or $\mathrm{AlF}$ but report tentative detections of $\mathrm{NaCl}$, both of which fall within our observed frequency range at $247.2397 \mathrm{GHz}$ for the $(19 \rightarrow 18)$ line and $260.2231 \mathrm{GHz}$ for the $(20 \rightarrow 19)$ line.

The line at $247.2397 \mathrm{GHz}$ falls on the edge of a band in our data; however a clear partial detection is visible, both in the spectrum and the channel maps. After correcting for the LSR velocity of $-23 \mathrm{~km} \mathrm{~s}^{-1}$ (Danilovich et al. 2014), we found that the $247.2397 \mathrm{GHz}$ line did not coincide with the emission we detect. A more likely carrier of the emission seen near this band edge is the ${ }^{30} \mathrm{SiO}(6 \rightarrow 5)$ line in the $v=4$ state at $247.244 \mathrm{GHz}$, owing to a better agreement with the systemic velocity of $\mathrm{W}$ Aql for the spatially compact emission of this line. In the case of the possible $\mathrm{NaCl}$ line at $260.2231 \mathrm{GHz}$, emission is present at this frequency; however we identify it as the $260.2248 \mathrm{GHz}$ line of $\mathrm{H}^{13} \mathrm{CN}$, with $J=3 \rightarrow 2$ in the $v_{2}=1$ excited bending vibrational level, which is in better agreement with the systemic velocity of W Aql. The $\mathrm{H}^{13} \mathrm{CN}$ line is also similar in brightness and appearance to the other component of the $l$-type doublet (with the same $J$ and $v_{2}$ values) seen at $258.9361 \mathrm{GHz}$, thereby confirming the 
Table 2. $\mathrm{HCl}$ and HF lines covered by Herschel/PACS towards W Aql.

\begin{tabular}{|c|c|c|c|c|}
\hline & $J^{\prime} \rightarrow J$ & $\begin{array}{c}\lambda \\
(\mu \mathrm{m})\end{array}$ & $\begin{array}{l}E_{\text {up }} \\
(\mathrm{K})\end{array}$ & $\begin{array}{c}\text { Flux } \\
\left(10^{-17} \mathrm{~W} \mathrm{~m}^{-2}\right)\end{array}$ \\
\hline $\mathrm{H}^{35} \mathrm{Cl}$ & $3 \rightarrow 2$ & 159.78 & 180 & $2.08 \pm 1.00$ \\
\hline $\mathrm{H}^{37} \mathrm{Cl}$ & $3 \rightarrow 2$ & 160.02 & 180 & $(0.77 \pm 1.02)$ \\
\hline $\mathrm{H}^{35} \mathrm{Cl}$ & $4 \rightarrow 3$ & 119.92 & 300 & $3.15 \pm 0.88$ \\
\hline $\mathrm{H}^{37} \mathrm{Cl}$ & $4 \rightarrow 3$ & 120.10 & 300 & $4.23 \pm 1.27$ \\
\hline $\mathrm{H}^{35} \mathrm{Cl}$ & $6 \rightarrow 5$ & 80.11 & 630 & ND \\
\hline $\mathrm{H}^{37} \mathrm{Cl}$ & $6 \rightarrow 5$ & 80.23 & 629 & ND \\
\hline $\mathrm{H}^{35} \mathrm{Cl}$ & $7 \rightarrow 6$ & 68.76 & 840 & ND \\
\hline $\mathrm{H}^{37} \mathrm{Cl}$ & $7 \rightarrow 6$ & 68.86 & 838 & ND \\
\hline $\mathrm{HF}$ & $2 \rightarrow 1$ & 121.70 & 178 & $\mathrm{H}_{2} \mathrm{O}$ blend \\
\hline $\mathrm{HF}$ & $3 \rightarrow 2$ & 81.22 & 355 & ND \\
\hline $\mathrm{HF}$ & $4 \rightarrow 3$ & 61.00 & 591 & ND \\
\hline
\end{tabular}

Notes. ND denotes a non-detection, parentheses indicate a tentative detection. Wavelengths and energies taken from Nolt et al. (1987) via the JPL Molecular Spectroscopy Database (Pickett et al. 1998).

assignment. An additional line of $\mathrm{NaCl},(17 \rightarrow 16)$ at $221.2601 \mathrm{GHz}$, was covered in our observations and by De Beck \& Olofsson (2020), but was not detected in either case.

\section{2. $\mathrm{HCl}$ and $\mathrm{HF}$}

A search of the literature revealed that, aside from the metal halides discussed above, the only other published detections of halogen-bearing molecules towards AGB stars are the hydrides $\mathrm{HCl}$ and $\mathrm{HF}$ towards the carbon star CW Leo (Cernicharo et al. 2010; Agúndez et al. 2011). The frequencies of both $\mathrm{HCl}$ and $\mathrm{HF}$ are well outside of the observing window of the ATOMIUM project. However, some transitions of $\mathrm{HCl}$ and $\mathrm{HF}$ were covered by the Herschel/PACS spectrograph (Pilbratt et al. 2010; Poglitsch et al. 2010) and observed towards several AGB stars (Groenewegen et al. 2011; Nicolaes et al. 2018). Although spectrally resolved transitions of $\mathrm{HCl}$ and $\mathrm{HF}$ were observed by Agúndez et al. (2011) towards CW Leo with Herschel/HIFI (de Graauw et al. 2010), all of these lines are outside of the frequency range at which W Aql was observed with Herschel/HIFI (Danilovich et al. 2014).

We searched the PACS spectrum of W Aql (Nicolaes et al. 2018) and found evidence of $\mathrm{H}^{35} \mathrm{Cl}$ and $\mathrm{H}^{37} \mathrm{Cl}$ emission in the $J=3 \rightarrow 2$ and $J=4 \rightarrow 3$ lines. In both cases, there is a partial overlap of the $\mathrm{H}^{35} \mathrm{Cl}$ and $\mathrm{H}^{37} \mathrm{Cl}$ lines, since the emission is not spectrally resolved by PACS. The $\mathrm{H}^{37} \mathrm{Cl}(4 \rightarrow 3)$ line is also known to be blended with $\mathrm{H}^{13} \mathrm{CN}(29 \rightarrow 28)$ at $120.12 \mu \mathrm{m}$ and $\mathrm{SiO}(58 \rightarrow 57)$ at $120.14 \mu \mathrm{m}$. Without spectrally resolved observations available, we cannot disentangle the contributions of these lines to the $\mathrm{H}^{37} \mathrm{Cl}(4 \rightarrow 3)$ line, but they are the most likely reason that the $\mathrm{H}^{37} \mathrm{Cl}$ appears brighter than the more abundant $\mathrm{H}^{35} \mathrm{Cl}$. We do not find clear detections of the $J=6 \rightarrow 5$ and $J=7 \rightarrow 6$ lines for either $\mathrm{HCl}$ isotopologue. However, we still compare the PACS spectrum in the region of these two lines to our model results (see Sect. 5.3.1), so that they can serve as upper limits. The details of these lines are listed in Table 2. We also include the measured fluxes of the detected lines, which were calculated by fitting Gaussians to the spectra.

We also searched the PACS spectrum for HF, for which three transitions fall within the observed PACS range: $J=2 \rightarrow 1$, $J=3 \rightarrow 2$, and $J=4 \rightarrow 3$ (see Table 2). The lowest energy of
Table 3. Stellar and circumstellar parameters of W Aql used in radiative transfer modelling.

\begin{tabular}{ll}
\hline \hline Distance & $395 \mathrm{pc}$ \\
Effective temperature, $T_{\star}$ & $2300 \mathrm{~K}$ \\
Dust optical depth ${ }^{(a)}$ at $10 \mu \mathrm{m}$ & 0.6 \\
${\text { Luminosity, } L_{\star}}_{\text {Stellar LSR velocity, } v_{\mathrm{LSR}}}$ & $-2300 \mathrm{~L}_{\odot}$ \\
Expansion velocity, $v_{\infty}$ & $16.5 \mathrm{~km} \mathrm{~s}^{-1}$ \\
Minimum velocity, $v_{0}$ & $3 \mathrm{~km} \mathrm{~s}^{-1}$ \\
Velocity index, $\beta$ & 2 \\
Dust condensation radius, $R_{\mathrm{in}}$ & $2 \times 10^{14} \mathrm{~cm}^{-6} M_{\odot} \mathrm{yr}^{-1}$ \\
Mass-loss rate, $\dot{M}$ & $3 \times 10^{-6}$
\end{tabular}

Notes. Parameters taken from Danilovich et al. (2014) and Ramstedt et al. (2017); ${ }^{(a)}$ see text for details of dust treatment for AlCl.

these lines, at $121.70 \mu \mathrm{m}$, is unfortunately blended with a bright ortho- $\mathrm{H}_{2} \mathrm{O}$ line at $121.721 \mu \mathrm{m}$. Higher spectral resolution observations would be needed to distinguish these two lines if the HF line is indeed present. We did not conclusively detect the two higher energy lines, although there is a faint line $(\sim 2 \sigma)$ that we tentatively attribute to $\mathrm{HF}(4 \rightarrow 3)$. As for $\mathrm{HCl}$, we include these lines in our model so that they might allow us to derive an upper limit for the abundance of HF.

\section{Modelling overview}

\subsection{Radiative transfer model}

Radiative transfer modelling was performed to determine the abundances and abundance distributions of our observed halide molecules. For the radiative transfer modelling of $\mathrm{AlCl}$ and AlF, we used a spherically symmetric model and the accelerated lambda iteration method (ALI, Rybicki \& Hummer 1991), previously used to model various molecules in the CSE of W Aql (Danilovich et al. 2014; Ramstedt et al. 2017; Brunner et al. 2018). For this study, we used the circumstellar parameters derived by Danilovich et al. (2014), including the assumption of silicate dust, and included the adjusted mass-loss rate found by Ramstedt et al. (2017). The velocity profile ${ }^{4}$ from Danilovich et al. (2014) was also used here:

$v(r)=v_{0}+\left(v_{\infty}-v_{0}\right)\left(1-\frac{R_{\mathrm{in}}}{r}\right)^{\beta}$,

with the parameters listed in Table 3 with the other circumstellar and stellar parameters. The gas kinetic temperature was derived in Danilovich et al. (2014) and Ramstedt et al. (2017) from CO radiative transfer modelling (see Schöier \& Olofsson 2001; Danilovich et al. 2014, for a discussion of the heating and cooling terms). As plotted in Fig. 4, we extended the kinetic temperature

4 We note our use of the expansion velocity implemented in previous radiative transfer models of $\mathrm{W}$ Aql, $v_{\infty}=16.5 \mathrm{~km} \mathrm{~s}^{-1}$ (Danilovich et al. 2014; Ramstedt et al. 2017; Brunner et al. 2018), which is smaller than the maximum velocity derived by Gottlieb et al. (2021), of $v_{\infty}=27.1 \mathrm{~km} \mathrm{~s}^{-1}$, partly due to low-intensity, high-velocity wings. As discussed in Danilovich et al. (2014), larger expansion velocities can be found for W Aql due to an auxiliary feature seen in the blue part of many molecular line profiles. This is routinely excluded from $1 \mathrm{D}$ radiative transfer modelling, an especially valid approach here, since we do not detect this feature or any other high-velocity components in the $\mathrm{AlCl}$ or AlF emission. 


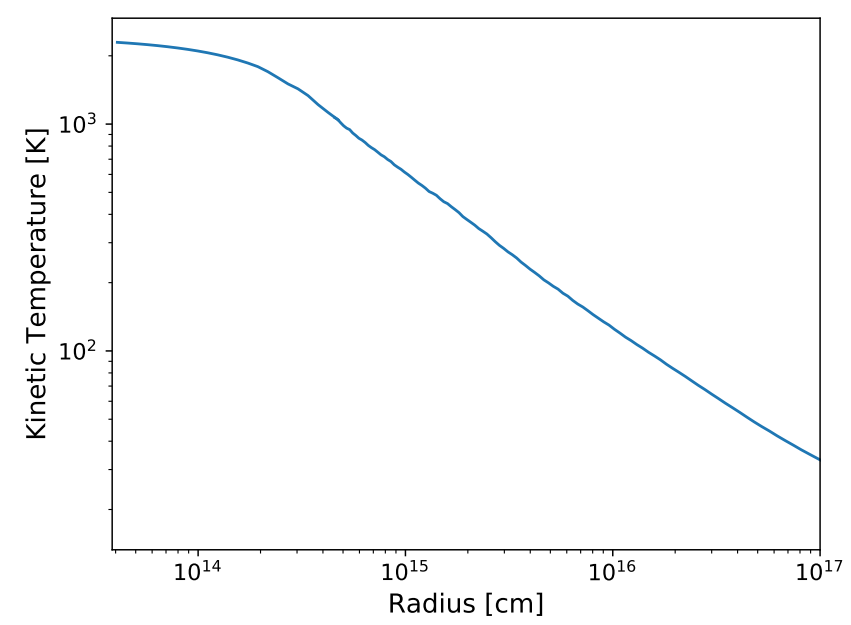

Fig. 4. Radial gas kinetic temperature profile used in our modelling of W Aql.

profile inwards such that the stellar effective temperature is not exceeded at the stellar surface.

For consistency with previous models, we used the distance for W Aql obtained by Danilovich et al. (2014) of 395 pc, which was found using a period-luminosity relation. This value is within the uncertainties of the parallax value from the Gaia Early Data Release 3 (Gaia Collaboration 2016, 2021), which gives a distance of $374 \pm 22 \mathrm{pc}$ after applying the corrections of Lindegren et al. (2021).

For $\mathrm{AlCl}$ and $\mathrm{AlF}$, we used a combination of spectral lines and azimuthally averaged radial profiles (centred on the continuum peak) to determine the molecular abundances and extents of the molecular envelopes. From earlier models of spatially resolved ALMA observations (such as Danilovich et al. 2019), we found that this method was best for finding the abundance distribution of the inner wind - most clearly seen in the ALMA azimuthal profile - while still constraining the outer wind. In general, fainter outer emission is often below the noise in the azimuthal profiles, but its signatures are more clearly seen in spectral lines, especially those with emitting regions farther out in the CSE, such as the $v=0 \mathrm{AlF}$ line.

Since the emitting regions for $\mathrm{AlCl}$ and $\mathrm{AlF}$ are quite different (see Fig. 3), there are some differences in how we treat the two molecules, particularly with respect to how dust is implemented in the models. AlF is treated similarly to other molecules in Brunner et al. (2018), using the dust characteristics obtained by Danilovich et al. (2014), since a significant portion of the AlF emission is detected outside the dust condensation radius $\left(R_{\text {in }}=2 \times 10^{14} \mathrm{~cm}\right.$, Danilovich et al. 2014).

From the ALMA observations, $\mathrm{AlCl}$ is predominantly found within 0 ' $^{\prime} 05\left(\approx 3 \times 10^{14} \mathrm{~cm}\right)$ of the star - that is, a significant portion of the $\mathrm{AlCl}$ is located within the dust condensation radius. One limitation of our ALI code is that it is not possible to simultaneously consider a dust-free inner region and a dusty outer region. To overcome this limitation, we run a dust-free model for $\mathrm{AlCl}$ but include an additional infrared radiation field, based on a blackbody with a temperature of $700 \mathrm{~K}$, which is close to the dust temperature at $3 \times 10^{14} \mathrm{~cm}$ in the Danilovich et al. (2014) model. This method is only used for $\mathrm{AlCl}$ since it is the most compact emission we see. For $\mathrm{HCl}$ and $\mathrm{HF}$, we start our model at $2 \times 10^{14} \mathrm{~cm}$ (i.e. the dust condensation radius), following the method in Danilovich et al. (2014), since we have no observational information on the behaviour of these molecules in the inner wind.
Although $\mathrm{AlF}$ is more extended than $\mathrm{AlCl}$, it is still relatively compact, compared with, for example, the molecules studied towards W Aql by Brunner et al. (2018), notably CS, SiS, and (quasi-thermal) $\mathrm{SiO}$, which extend out to $\sim 2^{\prime \prime}$ from the continuum peak. The predicted distributions of $\mathrm{HCl}$ and $\mathrm{HF}$ (see Sect. 4.3) are more similar in spatial extent to the molecules modelled by Brunner et al. (2018), so we include the overdensity derived in that study, increasing the density of the wind by a factor of five between $8 \times 10^{15} \mathrm{~cm}$ and $1.5 \times 10^{16} \mathrm{~cm}$. This overdensity, intended to represent the denser region of a spiral arm (see Ramstedt et al. 2017, for plots of the CO distribution), is outside of the region modelled for $\mathrm{AlF}$ or $\mathrm{AlCl}$, but may contribute to the flux of the $\mathrm{HCl}$ and $\mathrm{HF}$ lines, which extend past the overdense region.

\subsection{Molecular data}

A radiative transfer analysis of molecular observations requires a comprehensive list of molecular energy levels and transitions, including Einstein A coefficients and collisional (de-)excitation rates. We prefer to use collisional (de-)excitation rates measured or calculated for collisions between the target molecule and $\mathrm{H}_{2}$, but these are not always available - or are not available for our domain of interest - so some substitutions must be made. For most of the molecules studied here, we obtained the level and radiative information from the ExoMol database ${ }^{5}$ (Tennyson et al. 2016) and the collisional rates from a variety of sources detailed for each molecule in Appendix B. In particular, we use newly updated collisional rates for AlF, which cover the high temperatures seen for AGB CSEs and are described in detail in Appendix B.2.

Generally, collisional rates have only been calculated for the ground vibrational states of various molecules. Hence, we ensured that the rates used cover, at minimum, all levels that participate in our observed transitions in the ground vibrational state. Often, the available collisional rates are calculated for lower temperatures than seen in our models, for example, rates calculated for maximum kinetic temperatures of $300 \mathrm{~K}$ are common. In these cases, collisional rates for higher kinetic temperatures are linearly extrapolated in $\log -\log$ space from the given rates.

\subsection{Radial distribution of $\mathrm{HCl}$ and $\mathrm{HF}$ from chemical modelling}

In the absence of spatially resolved observations of $\mathrm{HCl}$ and $\mathrm{HF}$, and having only a small number of spectrally unresolved lines, we turned to predictions from chemical models to determine the $\mathrm{HCl}$ and $\mathrm{HF}$ abundance distributions. We use the 1D chemical kinetics model of Van de Sande et al. (2018) and the publicly available gas-phase only RATE12 reaction network ${ }^{6}$ (McElroy et al. 2013). The chemical model assumes the same mass-loss rate and stellar radius as the radiative transfer model, and a constant expansion velocity of $16.5 \mathrm{~km} \mathrm{~s}^{-1}$. The retrieved gas temperature profile (Fig. 4) was reproduced in the chemical model using two power-laws $(r<$ $2 \times 10^{14} \mathrm{~cm}, T=2100\left(r / 6.8 \times 10^{13}\right)^{-0.16} \mathrm{~K} ; r \geq 2 \times 10^{14} \mathrm{~cm}$, $\left.T=3350\left(r / 8.6 \times 10^{13}\right)^{-0.7} \mathrm{~K}\right)$.

To estimate the shape of the abundance profiles of $\mathrm{HF}$ and $\mathrm{HCl}$, we assumed an initial abundance of $\mathrm{F}$ and $\mathrm{Cl}$ corresponding to their solar abundance minus the inner abundances calculated

\footnotetext{
5 https://exomol.com

6 http://udfa . ajmarkwick . net/index . php?mode=downloads
} 

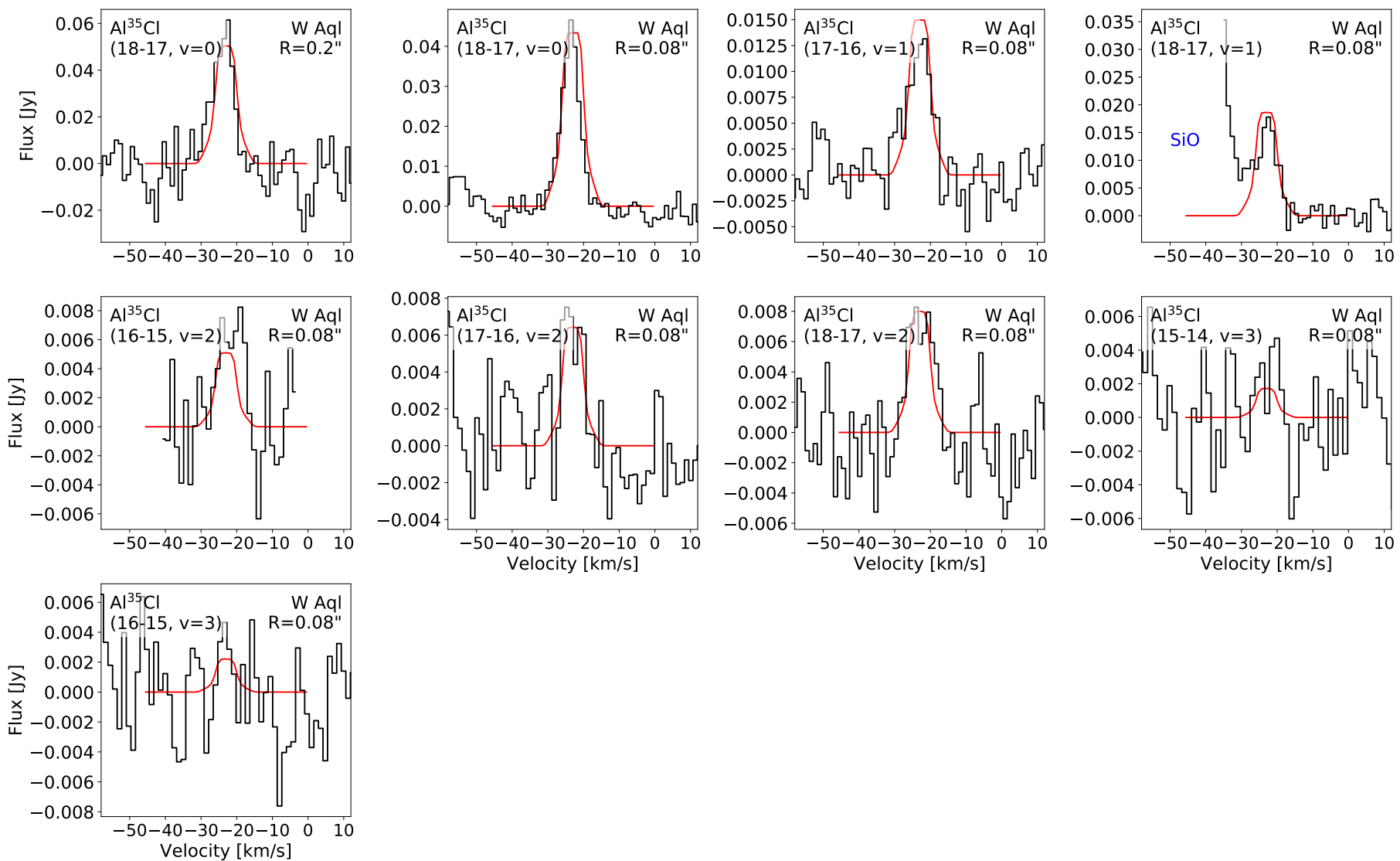

Fig. 5. Spectra of $\mathrm{Al}^{35} \mathrm{Cl}$ observed towards W Aql with ALMA (black histograms), with calculated line profiles (red curves) superposed on the observed profiles. Nearby lines of other molecules are labelled in blue.

for $\mathrm{AlCl}$ and $\mathrm{AlF}$ from early models (see Sects. 5.1 and 5.2). Both halogens are efficiently hydrogenised into $\mathrm{HF}$ and $\mathrm{HCl}$ at the start of the model. The other Cl- and F-bearing species included in the reaction network (which does not include $\mathrm{AlCl}$ or $\mathrm{AlF}$ ) play a negligible role. Our chemical model is expanded to include $\mathrm{Al}$ chemistry in Sect. 6.5, where we discuss the reactions important in the syntheses of $\mathrm{AlCl}$ and $\mathrm{AlF}$.

The shape of the predicted radial abundance distribution does not change with changes in initial $\mathrm{Cl}$ or $\mathrm{F}$ abundances. Hence, in our radiative transfer analysis, we find the best fitting model by scaling the radial abundance distribution until the model lines best reproduce the observed data. When finding upper limits on the abundance, we scale the radial abundance profile such that the model lines do not exceed the observed lines.

\section{Model results}

\section{1. $\mathrm{AlCl}$}

In attempting to fit the observed spectral lines of $\mathrm{Al}^{35} \mathrm{Cl}$, we started with a constant radial abundance profile to first determine the outer extent of the molecular envelope. To also fit the inner part of the azimuthally averaged radial profile, we needed to include a step down in abundance in the inner region. Our final best-fitting model, which agreed well with the radial profile and the spectral lines, had an inner abundance $f_{0}=8.5 \times 10^{-8}$, relative to $\mathrm{H}_{2}$, from the stellar surface out to $R_{\text {step }}=1.4 \times 10^{14} \mathrm{~cm}\left(\approx 3.6 R_{\star}\right)$. Then between $1.4 \times 10^{14} \mathrm{~cm}$ and an outer radius $R_{\max }=5 \times 10^{14} \mathrm{~cm}\left(\approx 13 R_{\star} \approx 00^{\prime} 08\right)$, we find a relative abundance of $1.7 \times 10^{-7}$. The model lines are plotted with the observed spectra in Fig. 5. We also plot the observed and modelled azimuthally averaged radial profiles in Fig. 6, with a residual plot showing the difference between the modelled and observed radial profile points. As can be seen in those plots, our model reproduces the observed data well. The main deviation between our model and the observations is the small 'tail' in the radial profile seen around $0 . ' 1$. We were not able to reproduce this with our model, possibly because it is caused by asymmetry in the detected emission (see Figs. 1 and 2). When we increased the outer radius of the model we reproduced the tail but failed to reproduce the shapes of the spectral line profiles. Using a larger outer radius in our model tended to increase the amount of emission coming from outside of $2 \times 10^{14} \mathrm{~cm}$, which is the region in which the gas accelerates. Emission from outside of $2 \times 10^{14} \mathrm{~cm}$ is generally broader and a model that reproduced the tail caused the calculated line profile to have a broad base with wings approximately $30 \%$ of the peak line flux. This is contrary to what is seen in the observations, especially for the $v=0(18 \rightarrow 17)$ line, which has the highest signal-to-noise ratio. Our best model includes part of the acceleration region, resulting in small wings on the model lines that are consistent with the observations.

For $\mathrm{Al}^{37} \mathrm{Cl}$ we used a similar modelling strategy. The lower abundance of the ${ }^{37} \mathrm{Cl}$ isotopologue leads to fainter lines and a noisier azimuthally averaged radial profile. Therefore, it is more difficult to fit a model to the radial profile. We find that a model with the same outer radius as for $\mathrm{Al}^{35} \mathrm{Cl}$ fits the data well. However, we do not require a step function to fit the radial profile of $\mathrm{Al}^{37} \mathrm{Cl}$. In fact, a test using a step function with the same $f_{0} / f_{1}$ 

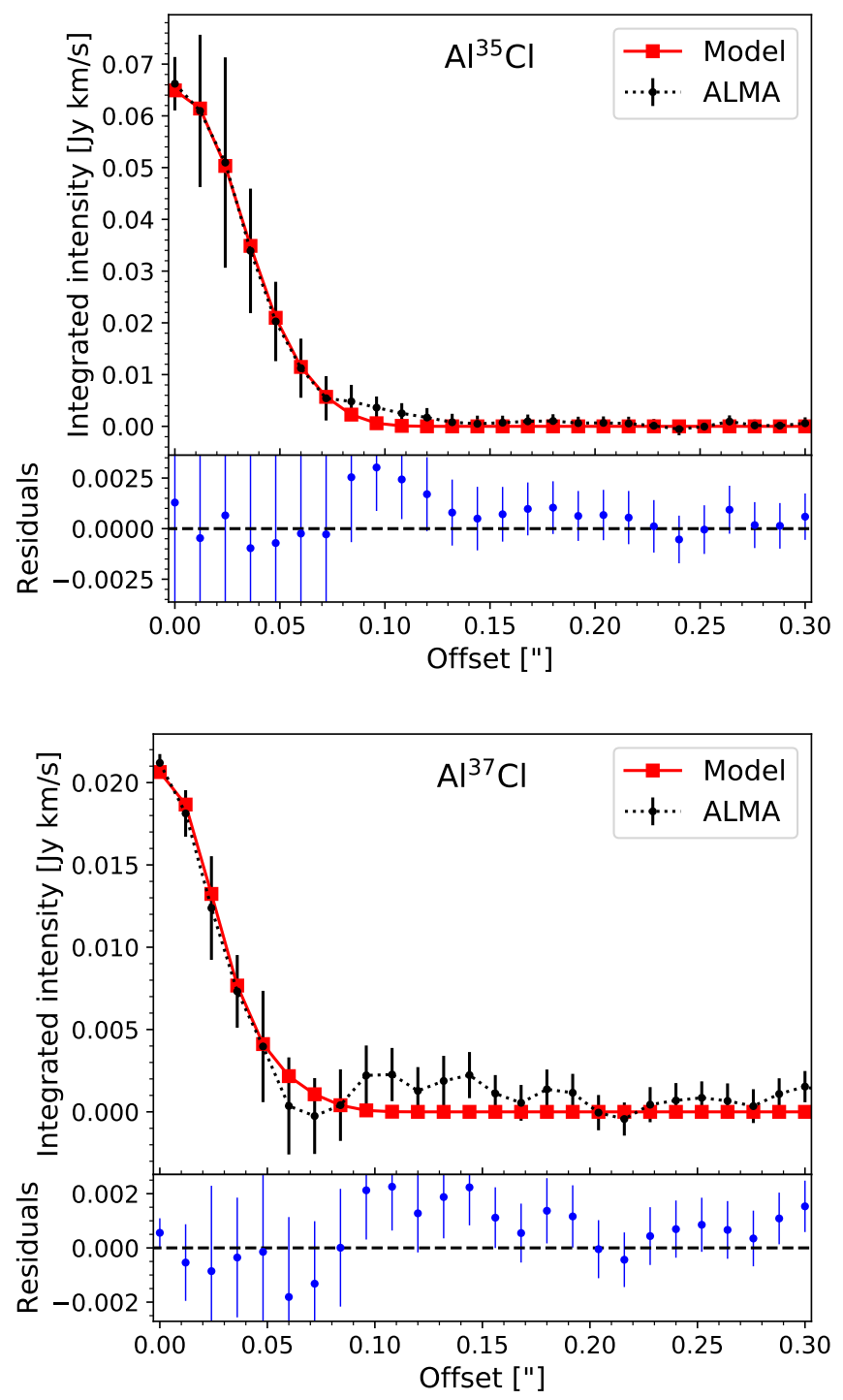

Fig. 6. Azimuthally averaged radial profiles extracted from ALMA $v=0$ lines of $\mathrm{AlCl}$ (black dotted lines and points with error bars) plotted with the corresponding modelled radial profiles (red lines and squares). Residuals are plotted in the lower panels, showing the difference between the observed and modelled lines, with error bars from the observations included.

as for $\mathrm{Al}^{35} \mathrm{Cl}$ did not reproduce the $\mathrm{Al}^{37} \mathrm{Cl}$ data as well. Instead we find the best model to have a constant abundance of $7 \times 10^{-8}$ relative to $\mathrm{H}_{2}$. If we require the abundance profile to have the same $f_{0} / f_{1}$ as for $\mathrm{Al}^{35} \mathrm{Cl}$, then we can only obtain models with higher $\chi^{2}$ values and, although we can find a model with a radial profile within most of the uncertainties of the observed radial profile, we are unable to reproduce the central point in this way. This is most likely a result of noise in the observations or could be because $\mathrm{Al}^{37} \mathrm{Cl}$ is present or excited in different clumps to $\mathrm{Al}^{35} \mathrm{Cl}$, leading to a difference in the azimuthally averaged profile (see Figs. 1 and 2). Since it best reproduces our data, we use the constant abundance model as our best fit model. The difference in the $\mathrm{Al}^{35} \mathrm{Cl}$ and $\mathrm{Al}^{37} \mathrm{Cl}$ distributions can be seen in the left and centre panels of Fig. 2, where we plot the zeroth moment maps of the $v=0$ lines for both isotopologues. The calculated line profiles from our best model are plotted with the observed spectra in Fig. 7 and the model and observed azimuthal profiles are plotted together in Fig. 6, along with a residual plot showing
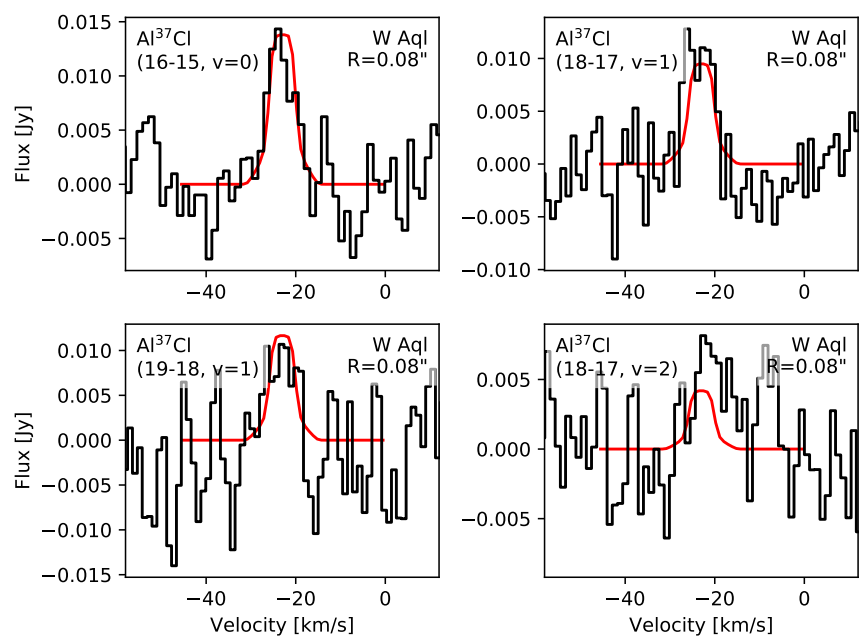

Fig. 7. Spectra of $\mathrm{Al}^{37} \mathrm{Cl}$ observed towards W Aql with ALMA (black histograms), with calculated line profiles (red curves) superposed on the observed profiles.

the difference between the modelled and observed radial profile points. Our results are tabulated in Table 4.

Since we do not find the same shape for the $\mathrm{Al}^{35} \mathrm{Cl}$ and $\mathrm{Al}^{37} \mathrm{Cl}$ abundance distributions, we are unable to determine a single ${ }^{35} \mathrm{Cl} /{ }^{37} \mathrm{Cl}$ ratio across the entire emitting region. In the inner region of our model (inside $1.7 \times 10^{14} \mathrm{~cm}$ ) we find $\mathrm{Al}^{35} \mathrm{Cl} / \mathrm{Al}^{37} \mathrm{Cl}=1.2$ and in the outer region we find it is twice as high: $\mathrm{Al}^{35} \mathrm{Cl} / \mathrm{Al}^{37} \mathrm{Cl}=2.4$. However, given the uncertainties of our observations (see Fig. 6 and Sect. 5.4), it is possible that the difference in these ratios may actually be less than a factor of two. Since the outer region represents a larger total volume of our circumstellar model, we adopt this value as the ratio for ${ }^{35} \mathrm{Cl} /{ }^{37} \mathrm{Cl}$.

\section{2. $A / F$}

For AlF we also started with a constant abundance model and then introduced a step function to fit the observed data. The azimuthally averaged radial profile (Fig. 8) has a bright inner component out to $\sim 0$.' 2 , then what looks like an extended plateau from $\sim 0$.' 2 to $\sim 0 . ' 5$. These features roughly correspond to the central inner region and the more diffuse emission seen in the zeroth moment map in Fig. 3. Since the emission seen in Fig. 3 is not centred on the star, our spherically symmetric model is unable to reproduce it perfectly. Also, as noted in Sect. 4.1, we include dust in the AlF model as described in Danilovich et al. (2014), since the majority of the AlF emission comes from beyond the dust condensation radius. Although we assume silicate dust opacities in our model (based on the results of Danilovich et al. 2014), W Aql does not have strong silicate features in its infrared spectrum (see Hony et al. 2009, and discussion in Sect 6.1.2). Hence, we also tested an AlF model with amorphous carbon dust (Suh 2000) in place of silicate dust. Using silicate dust, we find the best model with an abundance of $f_{0}=1 \times 10^{-7}$ relative to $\mathrm{H}_{2}$ in the inner region, with a step down at $R_{\text {step }}=6 \times 10^{14} \mathrm{~cm}\left(\approx 0 . ' 1 \approx 15.6 R_{\star}\right)$ to $f_{1}=4 \times 10^{-8}$ relative to $\mathrm{H}_{2}$, and the outer radius of the model at $R_{\text {max }}=3.5 \times 10^{15} \mathrm{~cm}\left(\approx 0{ }^{\prime} \cdot 6 \approx 90 R_{\star}\right)$. Using a model with amorphous carbon dust instead, we find a slightly lower inner abundance of $f_{0}=7.2 \times 10^{-8}$ but the same values for $R_{\text {step }}, f_{1}$, and $R_{\max }$. This difference, of less than $30 \%$, is most likely owing to radiative pumping, since the term energies of the AlF 
Table 4. Detailed model results for W Aql.

\begin{tabular}{cccccc}
\hline \hline Molecule & $R_{\text {in }}$ & $\begin{array}{c}f_{0} \\
\text { Rel. to } \mathrm{H}_{2}\end{array}$ & $\begin{array}{c}R_{\text {step }} \\
(\mathrm{cm})\end{array}$ & $\begin{array}{c}f_{1} \\
\text { Rel. to } \mathrm{H}_{2}\end{array}$ & $\begin{array}{c}R_{\max } \\
(\mathrm{cm})\end{array}$ \\
\hline $\mathrm{Al}^{35} \mathrm{Cl}$ & $R_{\star}$ & $8.5 \times 10^{-8}$ & $1.4 \times 10^{14}$ & $1.7 \times 10^{-7}$ & $5 \times 10^{14}$ \\
$\mathrm{Al}^{37} \mathrm{Cl}$ & $R_{\star}$ & $7 \times 10^{-8}$ & $\ldots$ & $7 \times 10^{-8}$ & $5 \times 10^{14}$ \\
$\mathrm{AlF}$ & $R_{\star}$ & $1 \times 10^{-7}$ & $6 \times 10^{14}$ & $4 \times 10^{-8}$ & $3.5 \times 10^{15}$ \\
$\mathrm{H}^{35} \mathrm{Cl}$ & $2 \times 10^{14} \mathrm{~cm}$ & $6.8 \times 10^{-8}$ & $\ldots$ & $\ldots$ & $1.6 \times 10^{16(*)}$ \\
$\mathrm{H}^{37} \mathrm{Cl}$ & $2 \times 10^{14} \mathrm{~cm}$ & $2.9 \times 10^{-8}$ & $\ldots$ & $\ldots$ & $1.6 \times 10^{16(*)}$ \\
$\mathrm{HF}$ & $2 R_{\star}$ & $1 \times 10^{-8}$ & $\ldots$ & $\ldots$ & $4 \times 10^{16(*)}$ \\
\hline
\end{tabular}

Notes. ${ }^{(*)}$ For $\mathrm{HCl}$ and $\mathrm{HF}$ we give $R_{10}$, the radius at which the abundance has dropped to $10 \%$ of $f_{0}$, in place of the model outer radius.

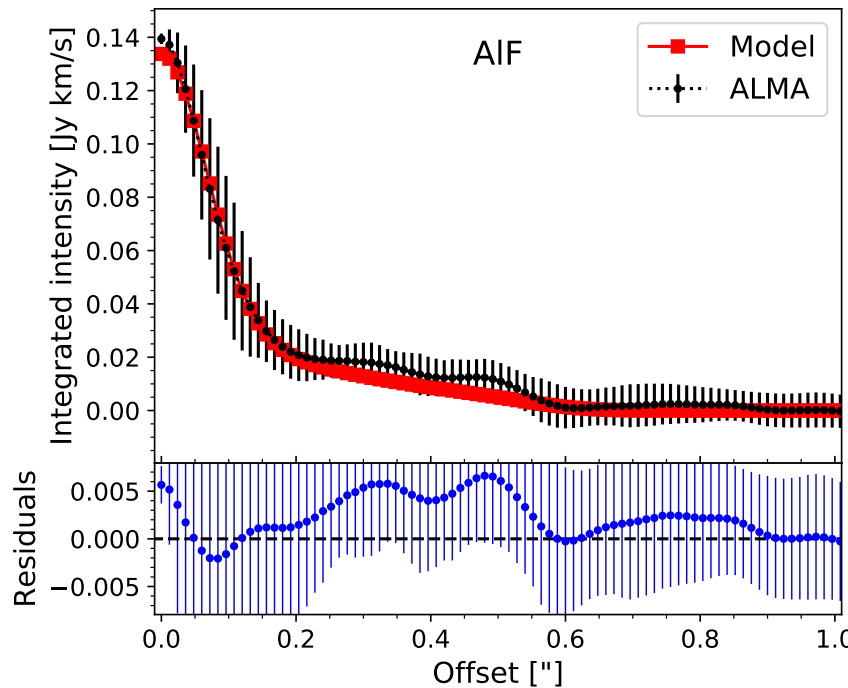

Fig. 8. Azimuthally averaged radial profile of AlF, extracted from the ALMA $(7 \rightarrow 6) v=0$ line (black dotted lines and points with error bars) plotted with the corresponding modelled radial profiles (red lines and squares). Residuals are plotted in the lower panels, showing the difference between the observed and modelled lines, with error bars from the observations included.

vibrational levels (12.6 $\mu \mathrm{m}$ for $v=1$ up to $2.2 \mu \mathrm{m}$ for $v=6$ ) overlap with the wavelengths for which dust (re)radiation contributes significantly to the radiation field. In the absence of a detailed characterisation of S-type AGB dust and for consistency with our other results, and previous studies of $\mathrm{W}$ Aql (Danilovich et al. 2014; Ramstedt et al. 2017; Brunner et al. 2018), we preferentially refer to the model results for silicate dust, unless otherwise specified.

The calculated line profiles for the silicate dust model are plotted with the observed lines in Fig. 9. The narrow central component and broader wings of the line profiles are the result of the velocity profile (Eq. (1)) and are a good fit to the observed spectral lines. As can also be seen in Fig. 9, the model predicts a very faint $v=1$ line for AlF, with flux lower than the noise of the observed spectrum ${ }^{7}$. The model and observed radial profiles are plotted together in Fig. 8, with a residual plot showing the difference between the modelled and observed radial profile points. The plateau part of the model radial profile mainly fits within the error bars of the observed radial profile, the discrepancy arising from the lack of spherical symmetry in the data. The innermost

\footnotetext{
7 This general result is unchanged for a model using amorphous carbon
} dust.
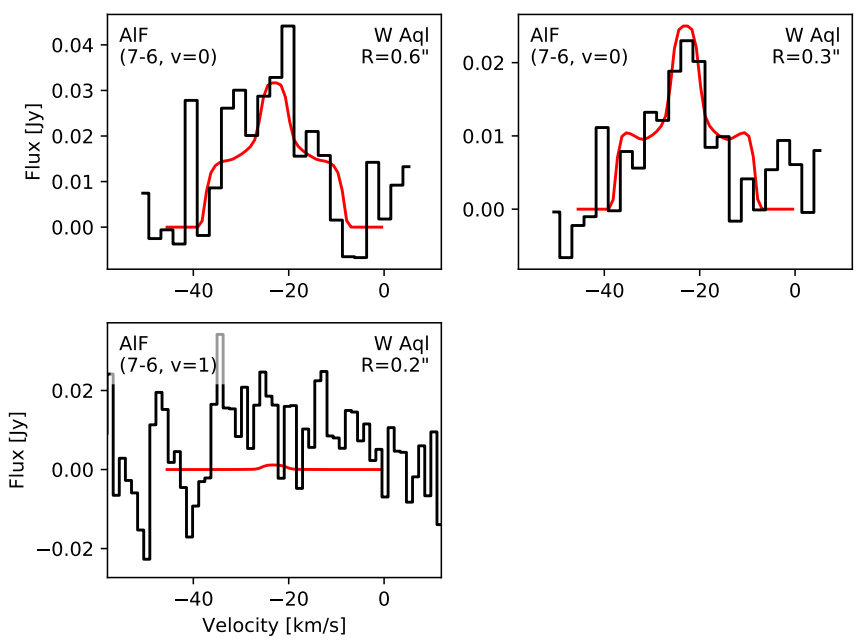

Fig. 9. Spectra of AlF observed towards W Aql with ALMA (black histograms), with calculated line profiles (red curves) superposed on the observed profiles.

few points are underpredicted by $7 \%$ or less and were not notably improved by adding a third step up in abundance in innermost regions.

We note that the relative abundance in the inner region of our AlF model exceeds the solar abundance of F $\left(7.2 \times 10^{-8}\right.$ Asplund et al. 2009) by almost $40 \%$. This is discussed in more detail in Sect. 6.3.

\subsection{Models of PACS observations}

The PACS spectrum of W Aql (Figs. 10 and 11) is noisiest in the $80 \mu \mathrm{m}$ region (the B2B band, see Poglitsch et al. 2010, for band ranges $)$; therefore, the $\mathrm{HCl}(6 \rightarrow 5)$ and the $\mathrm{HF}(3 \rightarrow 2)$ lines are not tightly constrained by the data. Although the $\mathrm{HCl}$ and $\mathrm{HF}$ lines in the $60 \mu \mathrm{m}$ region (the B2A band) are not formally detected above the noise, there are suggestive tentative detections of both of the $\mathrm{HCl}(7 \rightarrow 6)$ lines and the $\mathrm{HF}(4 \rightarrow 3)$ line at the expected wavelengths. We use these features as upper limits for our models.

\subsection{1. $\mathrm{HCl}$}

Taking the abundance distribution calculated from the chemical model described in Sect. 4.3, we scaled the abundance by a constant factor until we found a model that best fit our data without overpredicting any of our undetected lines (Table 2). We also fixed the $\mathrm{H}^{35} \mathrm{Cl} / \mathrm{H}^{37} \mathrm{Cl}$ abundance ratio to 2.4 , based on the outer 

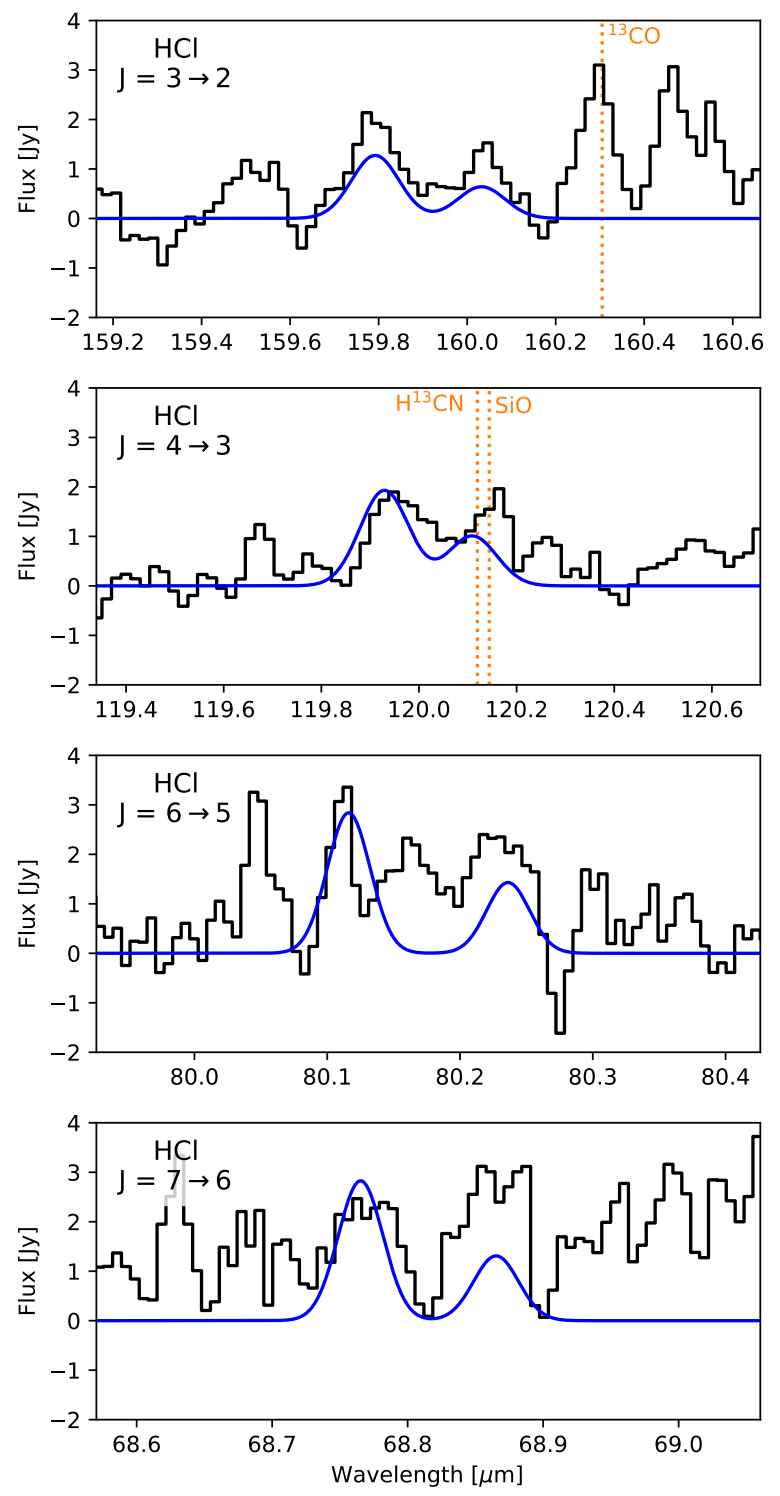

Fig. 10. PACS spectra (black histograms) and model results (blue curves) for $\mathrm{HCl}$ towards $\mathrm{W}$ Aql. For each pair of lines, $\mathrm{H}^{35} \mathrm{Cl}$ is shown on the left since it has the shorter wavelength and $\mathrm{H}^{37} \mathrm{Cl}$ is on the right, with the longer wavelength. Some known nearby and blended lines are indicated in orange (but not all nearby lines have been identified).

$\mathrm{Al}^{35} \mathrm{Cl} / \mathrm{Al}^{37} \mathrm{Cl}$ ratio (which is found in a region overlapping with the assumed inner part of the $\mathrm{HCl}$ distribution).

Our best fitting model had an inner $\mathrm{H}^{35} \mathrm{Cl}$ abundance of $6.8 \times 10^{-8}$, relative to $\mathrm{H}_{2}$, and an inner $\mathrm{H}^{37} \mathrm{Cl}$ abundance of $2.9 \times 10^{-8}$. Although the model was fit to the detected lines (see Table 2), the uncertainties inherent in the PACS data, particularly with regards to possible line blends, mean that these results should be considered upper limits. The model results, convolved with the PACS spectral resolution, are shown with the observed spectra in Fig. 10.

\subsubsection{HF}

No lines of HF were clearly detected in the PACS spectrum of W Aql. Nevertheless, we use the same method as for $\mathrm{HCl}$, scaling the abundance distribution derived from the chemical model described in Sect. 4.3. For consistency with our $\chi$ Cyg results (see Sect. 6.1.1 and Appendix C), we set the inner radius for HF as $R_{\text {in }}=2 R_{\star}$. We find an upper limit on the HF abundance of $\leq 1 \times 10^{-8}$ relative to $\mathrm{H}_{2}$.

A plot of our HF model, convolved to the PACS spectral resolution, is shown in Fig. 11, with the observed spectra. For the HF $(2 \rightarrow 1)$ line, which is blended with the $\mathrm{H}_{2} \mathrm{O}$ line at $121.721 \mu \mathrm{m}$, we use the $\mathrm{H}_{2} \mathrm{O}$ model intensity from Danilovich et al. (2014) as a proxy for the $\mathrm{H}_{2} \mathrm{O}$ contribution to the observed PACS line (shown in grey in Fig. 11). The sum of our HF model line and the $\mathrm{H}_{2} \mathrm{O}$ line is in good agreement with the line seen in the PACS spectrum.

\subsection{Model uncertainties}

The formal uncertainties on our models for a $90 \%$ confidence interval are around $20 \%$ for $\mathrm{AlCl}$ and $5 \%$ for $\mathrm{AlF}$, when $\chi^{2}$ statistics are calculated primarily from the radial profiles. However, our 1D model cannot account for the 3D effects that produce deviations from spherical symmetry, especially in the case of AlF (see Fig. 3). This means that our actual uncertainties are much larger than the formal uncertainties and cannot easily be quantified. This holds even though the error bars on the azimuthally averaged radial profiles (shown in Figs. 6 and 8) take into account deviations from azimuthal symmetry in the distribution of emission, as well as stochastic noise.

The uncertainties on the $\mathrm{HCl}$ and $\mathrm{HF}$ model abundances are also larger than can be easily quantified from the formal errors. Owing to the low spectral resolution of PACS, there is substantial uncertainty as to whether the lines of interest are blended with nearby lines (for example, the $\mathrm{H}^{35} \mathrm{Cl}$ and $\mathrm{H}^{37} \mathrm{Cl}(3 \rightarrow 2)$ lines are separated by almost $3 \mathrm{GHz}$, despite overlapping in their wings at the PACS resolution). This uncertainty can only be alleviated with spectrally resolved observations.

An additional source of uncertainty in our models, particularly for the case of AlF (see Sect. 5.2), is the choice of dust type. We primarily use silicate dust for consistency with Danilovich et al. (2014), who also performed SED modelling to derive the dust optical depths. When we tested amorphous carbon dust with our AlF model, we used the same optical depth for the dust since running new SED models is beyond the scope of the present study. However, Hony et al. (2009) found that dust around S-type stars bears some similarities to M-type dust with some variation in features (see Sect. 6.1.2).

Another source of uncertainty comes from our extrapolation of the CSE model of Danilovich et al. (2014) inwards towards the star. Our model does not consider gas infall or stellar pulsations, which are likely to have an effect on material in the innermost regions of the CSE. The gas number density of our model in these inner regions is extrapolated inwards following the power law $n=\dot{M} /\left(4 \pi r^{2} v(r)\right)$, which assumes an expanding CSE with a constant mass-loss rate. However, recent models of the warm molecular layer close to AGB stars (including W Aql, Khouri et al. 2016) have yielded higher number densities (by around an order of magnitude) in this region. Since part of the $\mathrm{AlCl}$ and AlF emission is thought to come from this innermost warm molecular layer, this adds further uncertainty to the inner $2-3 R_{\star}$ $(\sim 15$ mas $)$ of our models.

\section{Discussion}

\subsection{Halogens towards other AGB stars}

We searched the literature and in Table 5 we have compiled the measured abundances of the aluminium and hydrogen halides for AGB stars. In the following subsections, 

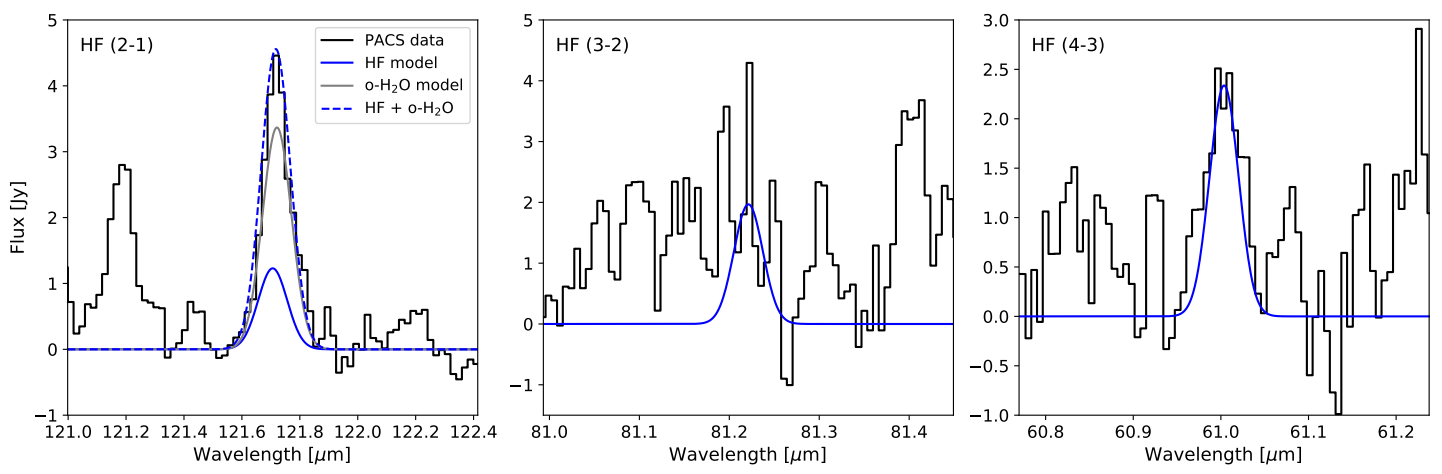

Fig. 11. PACS spectra (black histograms) and model results (blue curves) for HF towards W Aql. The HF $(2 \rightarrow 1)$ line is dominated by a blend with the $\mathrm{o}-\mathrm{H}_{2} \mathrm{O}$ line at $121.721 \mu \mathrm{m}$, which is plotted in grey. See text for details.

Table 5. Abundances relative to $\mathrm{H}_{2}$ of halide molecules found towards AGB stars.

\begin{tabular}{|c|c|c|c|c|c|c|}
\hline \multirow[b]{2}{*}{ Molecule } & \multicolumn{3}{|c|}{ S-type stars } & \multirow{2}{*}{$\begin{array}{c}\text { Carbon star } \\
\text { CW Leo }\end{array}$} & \multicolumn{2}{|c|}{ Oxygen-rich stars } \\
\hline & W Aql & $\chi$ Cyg & $\mathrm{R}$ And & & R Dor & IK Tau \\
\hline $\mathrm{AlCl}$ & $1.6 \times 10^{-7}-2.4 \times 10^{-7}$ & . & . & $7 \times 10^{-8}$ & $2.5 \times 10^{-8}$ & $9 \times 10^{-10}$ \\
\hline AlF & $1 \times 10^{-7}-4 \times 10^{-8}$ & $\ldots$ & & $1 \times 10^{-8}$ & $\ldots$ & $\ldots$ \\
\hline $\mathrm{HCl}$ & $9.7 \times 10^{-8}$ & $6.5 \times 10^{-8}$ & $1.5 \times 10^{-8}$ & $1 \times 10^{-7}$ & $\ldots$ & $\ldots$ \\
\hline $\mathrm{HF}$ & $\leq 1 \times 10^{-8}$ & $1.2 \times 10^{-8}$ & $\ldots$ & $8 \times 10^{-9}$ & $\ldots$ & $\ldots$ \\
\hline Ref. & This work & This work ${ }^{(a)}$ & 1 & 2 & 3 & 3 \\
\hline
\end{tabular}

Notes. For comparison, the solar abundances of $\mathrm{F}$ and $\mathrm{Cl}$, relative to $\mathrm{H}_{2}$, are $7.2 \times 10^{-8}$ and $6.3 \times 10^{-7}$, respectively (Asplund et al. 2009). ${ }^{(a)}$ See Sect. 6.1.1 and Appendix C.

References. (1) Yamamura et al. (2000); (2) Agúndez et al. (2011, 2012); (3) Decin et al. (2017).

we discuss the abundances of these molecules for individual sources, grouped into S-type AGB stars (Sect. 6.1.1), carbon stars (Sect. 6.1.2), and oxygen-rich AGB stars (Sect. 6.1.3). We also touch on mid-infrared observations of $\mathrm{HCl}$ and $\mathrm{HF}$ in Sect. 6.1.4.

\subsubsection{S-type AGB stars}

No halide molecules (i.e. $\mathrm{AlF}, \mathrm{AlCl}, \mathrm{NaCl}, \mathrm{KCl}$ ) were detected towards $\pi^{1}$ Gru, the only other S-type star in the ATOMIUM survey. This may be partly because the unusual torus + bipolar structure of the CSE of $\pi^{1}$ Gru (Doan et al. 2017; Homan et al. 2020) could make it harder to detect less abundant molecules or might even interfere with molecule formation. We are not aware of any other detections of halide molecules towards S-type stars with ALMA.

PACS spectra were taken for only three S-type AGB stars: W Aql, $\pi^{1}$ Gru and $\chi$ Cyg (Groenewegen et al. 2011; Nicolaes et al. 2018). We checked the PACS spectra of $\pi^{1} \mathrm{Gru}$ and $\chi$ Cyg for the signatures of $\mathrm{HCl}$ and $\mathrm{HF}$. We found evidence of $\mathrm{HCl}$ and $\mathrm{HF}$ towards $\chi \mathrm{Cyg}$ and, tentatively, $\mathrm{HF}$ towards $\pi^{1}$ Gru. The aforementioned complex circumstellar structure of $\pi^{1}$ Gru is such that it cannot be modelled under the assumption of spherical symmetry (see also the unusual CO line structure presented in Danilovich et al. 2015b). However, we are able to run radiative transfer models models for $\chi$ Cyg $-\mathrm{q}$ see details in Appendix C. We find inner relative abundances of $4.6 \times 10^{-8}$ and $1.9 \times 10^{-8}$ for $\mathrm{H}^{35} \mathrm{Cl}$ and $\mathrm{H}^{37} \mathrm{Cl}$, respectively (assuming, in the absence of other data, the same ${ }^{35} \mathrm{Cl} /{ }^{37} \mathrm{Cl}$ ratio as for $\mathrm{W} A q l$ ), and $1.2 \times 10^{-8}$ for HF.
The $\mathrm{HCl}$ abundances are around 50\% higher for $\mathrm{W}$ Aql than for $\chi$ Cyg, and the HF abundance is $20 \%$ higher for $\chi$ Cyg than the upper limit found for W Aql. For both molecules, these differences are within the observational uncertainties of the PACS data, especially for the very weak lines detected towards W Aql. The relative proximity of $\chi$ Cyg $(150 \mathrm{pc}$, Schöier et al. 2011) results in lines with higher signal to noise ratios in the PACS spectrum (see Fig. C.1), despite the similar abundances between the two stars, and the lower mass-loss rate of $\chi$ Cyg $\left(7 \times 10^{-7} M_{\odot} \mathrm{yr}^{-1}\right.$, Schöier et al. 2011). From the available data, we are unable to conclude whether the abundances of $\mathrm{HCl}$ and $\mathrm{HF}$ have a mass-loss rate dependence, as has been seen for some other molecules (e.g. SiO González Delgado et al. 2003; Ramstedt et al. 2009). Following similar arguments, we are also unable to conclude whether the abundances of $\mathrm{HCl}$ and $\mathrm{HF}$ depend on the $\mathrm{C} / \mathrm{O}$ ratio of the star, despite $\mathrm{W}$ Aql (S6/6e) and $\chi$ Cyg (S8/1) being categorised as one grade away from the SC and MS classifications, respectively (Turnshek et al. 1985; Gray et al. 2009; Danilovich et al. 2015a). A larger sample is required to draw firmer conclusions.

\subsubsection{Carbon stars}

CW Leo (IRC+10216) is the closest carbon star and all the halide molecules mentioned thus far have been detected in its CSE. It is also the only other AGB star for which abundances of $\mathrm{HCl}$ and HF have been calculated from radiative transfer modelling. Agúndez et al. (2011) found an inner abundance of $8 \times 10^{-8}$ relative to $\mathrm{H}_{2}$ for $\mathrm{H}^{35} \mathrm{Cl}$ (and an $\mathrm{H}^{35} \mathrm{Cl} / \mathrm{H}^{37} \mathrm{Cl}$ ratio of 3.3) and of $8 \times 10^{-9}$ for $\mathrm{HF}$. The $\mathrm{HCl}$ abundance for CW Leo is comparable to that found for $\mathrm{W}$ Aql but $50 \%$ higher than that found for $\chi \mathrm{Cyg}$. 


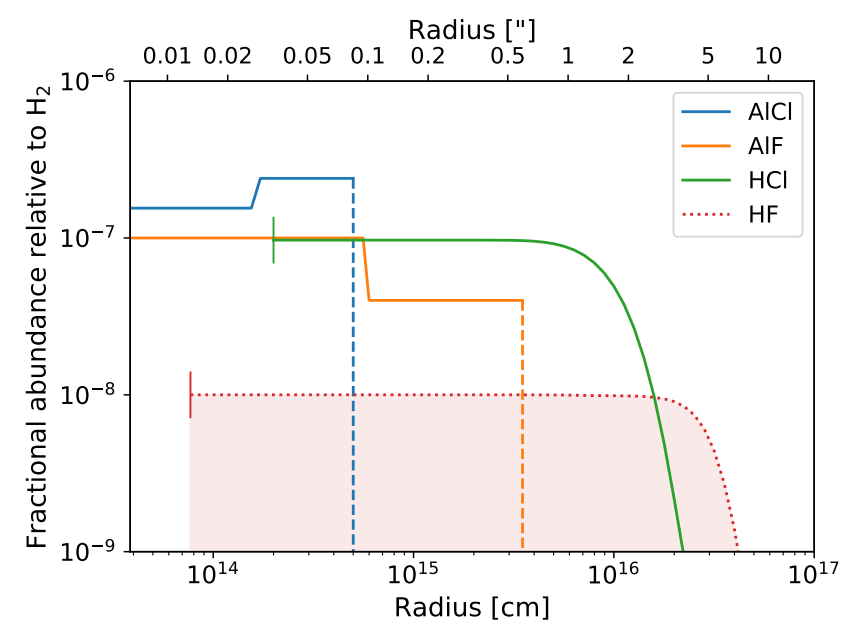

Fig. 12. Abundance profiles for the halide molecules modelled here. Abundances for ${ }^{35} \mathrm{Cl}$ and ${ }^{37} \mathrm{Cl}$ isotopologues have been combined. Vertical dashed lines indicate the outer radius of the corresponding model and short vertical lines indicate the inner radii of the $\mathrm{HCl}$ and $\mathrm{HF}$ models. The dotted curve and corresponding shading indicates an upper limit abundance for HF. See text for details.

Conversely, the HF abundance is comparable to the upper limit found for W Aql but 33\% lower than that found for $\chi$ Cyg. The observed abundances towards CW Leo of these two molecules are a factor of $\sim 5$ lower for $\mathrm{HCl}$ and an order of magnitude higher for $\mathrm{HF}$ than the predictions made by the chemical kinetics model of Cherchneff (2012), which considers shocks for models of the inner $5 R_{\star}$ of the CSE.

Based on observations with the IRAM $30 \mathrm{~m}$ telescope (with beam sizes ranging from $7^{\prime \prime}$ to $30^{\prime \prime}$ ), abundances of the halide molecules $\mathrm{AlCl}, \mathrm{AlF}, \mathrm{NaCl}$, and $\mathrm{KCl}$, were modelled by Agúndez et al. (2012) for CW Leo. In the data presented by Agúndez et al. (2012), there are no detections of any vibrationally excited lines for any of these halide molecules (though $\mathrm{NaCl}$ in the $v=1$ state was subsequently seen by Quintana-Lacaci et al. 2016, using ALMA). Despite $\mathrm{AlCl}$ and AlF having significantly lower dipole moments than $\mathrm{NaCl}$ and $\mathrm{KCl}$, Agúndez et al. (2012) saw similar line strengths for all four halogen-bearing species. They conclude that this is due to the higher abundances of $\mathrm{AlCl}$ and $\mathrm{AlF}$ compared with $\mathrm{NaCl}$ and $\mathrm{KCl}$, which is borne out by their model results $\left(7 \times 10^{-8}\right.$ and $1 \times 10^{-8}$ for $\mathrm{AlCl}$ and $\mathrm{AlF}$ compared with $1.8 \times 10^{-9}$ and $5 \times 10^{-10}$ for $\mathrm{NaCl}$ and $\mathrm{KCl}$ ). The most striking difference between their model results for CW Leo and ours for W Aql is the much larger molecular envelope sizes of $\mathrm{AlCl}$ and AlF towards CW Leo. Although Agúndez et al. (2012) do not list specific parameters for the sizes of the envelopes, from their Fig. 13 it can be seen that $\mathrm{AlCl}$ is present at an appreciable abundance $\left(\sim 1 \times 10^{-9}\right.$ relative to $\left.\mathrm{H}_{2}\right)$ out to $\sim 2 \times 10^{16} \mathrm{~cm}$ and AlF out to $\sim 4 \times 10^{16} \mathrm{~cm}$. This is much larger than our outer radii of $5 \times 10^{14} \mathrm{~cm}$ and $3.5 \times 10^{15} \mathrm{~cm}$ for AlCl and AlF. They also observe $\mathrm{AlCl}$ and $\mathrm{AlF}$ lines with expansion velocities around $14.5 \mathrm{~km} \mathrm{~s}^{-1}$, equal to the terminal gas expansion velocity used in their models. This is in contrast with what we see for W Aql, where $\mathrm{AlCl}$ is found in a region very close to the star (see Figs. 1 and 12) and the expansion velocity of $\mathrm{AlCl}$, as derived from the vibrational ground state, is around $4 \mathrm{~km} \mathrm{~s}^{-1}$ - that is, much lower than the terminal expansion velocity of $16.5 \mathrm{~km} \mathrm{~s}^{-1}$ (Danilovich et al. 2014) or the maximum velocity found by Gottlieb et al. (2021) (see Figs. 5 and 7).

Quintana-Lacaci et al. (2016) observed $\mathrm{Al}^{35} \mathrm{Cl}, \mathrm{Al}^{37} \mathrm{Cl}$, and AlF with ALMA towards CW Leo. In all cases, they found there was resolved out flux in the ALMA observations, when compared with spectra obtained from the IRAM $30 \mathrm{~m}$ telescope. $\mathrm{Al}^{37} \mathrm{Cl}$ was less affected than the other two species and, from the plots presented (Figs. 12 and 13 of Quintana-Lacaci et al. 2016), it can be seen that the $\mathrm{Al}^{37} \mathrm{Cl}$ extends out to $\sim 2^{\prime \prime}$ from the continuum peak. Assuming a distance of $130 \mathrm{pc}$ (Agúndez et al. 2012), this corresponds to $\sim 4 \times 10^{15} \mathrm{~cm}$, which is almost an order of magnitude larger than what we see for W Aql (see Fig. 12). Another difference we see from comparison to the Quintana-Lacaci et al. (2016) data is a lack of $v>0 \mathrm{AlCl}$ detections towards CW Leo, despite $v=1,2$ lines being present in the covered frequency range, and even though the $v=0 \mathrm{AlCl}$ lines in the CW Leo dataset have flux densities almost 18 times higher than our W Aql data, when measured in an equivalent aperture ${ }^{8}$. The absence of vibrationally excited lines could indicate different formation pathways if $\mathrm{AlCl}$ is not present as close to the star in the CSE of CW Leo compared with W Aql.

The AlF emission observed by Quintana-Lacaci et al. (2016) for the $(8 \rightarrow 7)$ transition is significantly resolved out by ALMA. Based on their observing setup, the authors estimate that smooth emission larger than 13-14" was filtered out. From their plot of the recovered AlF emission, it seems that the emission around the central $\sim 15 \mathrm{~km} \mathrm{~s}^{-1}$ was the most resolved out. Based on their estimate of the maximum resolvable scale, we can expect that AlF emission extends out to at least $6.5-7^{\prime \prime}$ from the star, assuming relatively symmetric emission. At a distance of $130 \mathrm{pc}$, this corresponds to $\sim 1.3 \times 10^{16} \mathrm{~cm}$ or about five times larger than our AlF model for W Aql.

Since the temperatures (agreement within $30 \mathrm{~K}$ ) and luminosities (CW Leo $17 \%$ more luminous) of the two stars are similar, we can conclude that the differences between $\mathrm{AlCl}$ and $\mathrm{AlF}$ distributions should mainly be because of the different chemical compositions and wind densities of the two CSEs (the latter dependent mainly on the mass-loss rates: $\dot{M}=2 \times 10^{-5} M_{\odot} \mathrm{yr}^{-1}$ for CW Leo compared with $\dot{M}=3 \times 10^{-6} M_{\odot} \mathrm{yr}^{-1}$ for W Aql). This in itself is an interesting result since the equilibrium models of Agúndez et al. (2020) predict very similar abundances between carbon stars and S-type stars for both $\mathrm{Cl}$ - and Al-bearing molecules.

Another possibility is that dust composition and interactions between gas and dust species could also contribute to the differences seen between W Aql and CW Leo. As a carbon star, CW Leo is surrounded by carbon-rich dust. As an S-type star, the dust around $\mathrm{W}$ Aql is less well understood. Hony et al. (2009) analyse infrared spectra from ISO/SWS for several AGB stars, with a focus on S-type stars. They note that there are weak features in the spectrum of W Aql (and other S stars) that are similar to but broader and less structured than the silicate and aluminium oxide features present in M-type AGB stars. Smolders et al. (2012) studied a large sample of S-type stars (not including W Aql) observed with Spitzer, and found that around half of them did not exhibit any alumina features. Although rotational lines of $\mathrm{AlO}$ were not detected towards W Aql, despite two lines in the ground vibrational state being covered by the ATOMIUM programme, and despite a lack of clear aluminium

\footnotetext{
8 The ALMA beam size and rms are $\sim 0 .^{\prime} 7,4 \mathrm{mJy}$ and 0 .' $^{\prime} 023,1 \mathrm{mJy}$ for the CW Leo data and our data, respectively. The $v=2$ line covered by both observations (considered since the $v=1$ is close to a brighter $\mathrm{SiO}$ line), has a total flux density of $13 \mathrm{mJy}$ over a few beam areas at the line peak for W Aql. The proportional flux density for the CW Leo data, when compared with the $v=0$ line, would be $23 \mathrm{mJy} / \mathrm{beam}$. This is just over $5 \sigma$ but it is possible for weak emission to be missed in masking for cleaning, especially in the Cycle 0 data with fewer antennas and less refined calibration.
} 
features in the infrared spectrum, it is possible that $\mathrm{AlCl}$ is incorporated into dust, hence explaining why it is seen only in regions close to the star. AlF may also be partly incorporated into dust, hence explaining the step down in abundance at approximately the same distance from the star as the outer edge of our $\mathrm{AlCl}$ model. Both the step down in $\mathrm{AlF}$ abundance and the outer radius of $\mathrm{AlCl}$ are within a factor of $\sim 3$ of the dust condensation radius. The persistence of $\mathrm{AlF}$ further out in the envelope, after $\mathrm{AlCl}$ has been destroyed, is likely to be at least partially due to the higher binding energy of AlF compared with $\mathrm{AlCl}\left(681 \mathrm{~kJ} \mathrm{~mol}^{-1}\right.$ for AlF and $507 \mathrm{~kJ} \mathrm{~mol}^{-1}$ for $\mathrm{AlCl}$, Curtiss et al. 2007). A more detailed discussion of chemistry is given in Sect. 6.5.

\subsubsection{Oxygen-rich AGB stars}

Aside from W Aql, in this study, and CW Leo, we have found no other published observations of AlF towards AGB stars. However, we note that AIF has been detected around several oxygen-rich ATOMIUM sources (Wallström et al., in prep.), which will be modelled in a future study. In the ATOMIUM sample, $\mathrm{AlCl}$ was also detected towards the oxygen-rich AGB star GY Aql, which will also be studied in a future publication.

$\mathrm{Al}^{35} \mathrm{Cl}$ has also been tentatively detected at relatively low abundances towards the oxygen-rich stars R Dor (low mass-loss rate, $\dot{M}=1.6 \times 10^{-7} M_{\odot} \mathrm{yr}^{-1}$ ) and IK Tau (higher mass-loss rate, $\dot{M}=5 \times 10^{-6} M_{\odot} \mathrm{yr}^{-1}$ ), as reported by Decin et al. (2017). For both stars, $\mathrm{Al}^{35} \mathrm{Cl}$ was found to be confined to the region close to the central star, similar to our results for W Aql (although our ALMA observations are at a higher spatial resolution and can put more stringent constraints on the $\mathrm{AlCl}$ emission region). Based on models that only considered rotational levels in the ground vibrational state of $\mathrm{Al}^{35} \mathrm{Cl}$, Decin et al. (2017) derived abundances are $2.5 \times 10^{-8}$ and $9 \times 10^{-10}$ relative to $\mathrm{H}_{2}$ for $\mathrm{R}$ Dor and IK Tau, respectively. These abundances are around one to two orders of magnitude lower than the $\mathrm{Al}^{35} \mathrm{Cl}$ abundance we found for $\mathrm{W}$ Aql. Gaseous $\mathrm{AlO}$ and $\mathrm{AlOH}$ have also been detected around the same two oxygen-rich stars (Decin et al. 2017; Danilovich et al. 2020), but at low enough abundances that we would not expect their presence to inhibit the production of $\mathrm{AlCl}$.

$\mathrm{NaCl}$ and $\mathrm{KCl}$ have been detected towards several oxygenrich stars, including some in the ATOMIUM sample, to be presented in a future study. Spectral scans of IK Tau, carried out using single-dish telescopes, detected several $\mathrm{NaCl}$ lines but no $\mathrm{KCl}$ lines (Milam et al. 2007; Velilla Prieto et al. 2017). A spectral scan of $\mathrm{R}$ Dor and IK Tau using ALMA found clumpy $\mathrm{NaCl}$ emission towards IK Tau (Decin et al. 2018), no $\mathrm{NaCl}$ emission towards $\mathrm{R}$ Dor, and no $\mathrm{KCl}$ towards either star, although De Beck \& Olofsson (2018) tentatively find $\mathrm{NaCl}$ towards $\mathrm{R}$ Dor in an APEX spectral scan. $\mathrm{NaCl}$ and $\mathrm{KCl}$ in the ground and several vibrationally excited states have been seen towards extreme OH/IR stars, oxygen-rich stars with high massloss rates $\left(\gtrsim 10^{-5} M_{\odot} \mathrm{yr}^{-1}\right)$, including $\mathrm{OH} 26+0.6$ (Justtanont et al. 2019) and $\mathrm{OH} 30.1-0.7$ (Danilovich et al., in prep.). Tentatively, it seems that there is a positive correlation between higher abundances of $\mathrm{NaCl}$ and $\mathrm{KCl}$ and higher mass-loss rates for oxygen-rich AGB stars, though this will be explored in more detail in a future study.

The chemical kinetics model of Gobrecht et al. (2016) considered shocks in the inner wind and included chloride species for a model based on IK Tau. They predict an average abundance for $\mathrm{HCl}$ of $3.8 \times 10^{-7}$ relative to $\mathrm{H}_{2}$, around 4 times higher than our S-star results. They also predict a very low abundance of $\mathrm{AlCl}$, with $3.8 \times 10^{-12}$ close to the stellar surface and $2.2 \times 10^{-10}$ at $6 R_{\star}$. This is lower than the Decin et al. (2018) observational result for IK Tau of $9 \times 10^{-10}$, by a factor of around four at $6 R_{\star}$ and by two orders of magnitude close to the stellar surface. This corresponds to 3 to 5 orders of magnitude lower than our W Aql results. In the Gobrecht et al. (2016) model, $\mathrm{NaCl}$ was a more significant carrier of $\mathrm{Cl}$ than $\mathrm{AlCl}$ but only reached $\sim 3 \%$ of the $\mathrm{HCl}$ abundance in the outer part of their model (at $9 R_{\star}$ ).

\subsubsection{Rovibrational observations of halides}

Thus far we have discussed observations of rotational transitions of halide molecules, but observations of rovibrational bands in the mid-infrared are also possible. Yamamura et al. (2000) report $\mathrm{HCl}$ lines in the spectrum of $\mathrm{R} \mathrm{And,} \mathrm{an} \mathrm{S-type} \mathrm{star} \mathrm{with} \mathrm{a} \mathrm{mod-}$ erately low mass-loss rate $\left(5.3 \times 10^{-7} M_{\odot} \mathrm{yr}^{-1}\right.$, Danilovich et al. $2015 b$ ). A rough comparison between their reported column density and $\mathrm{CO}$ gives an approximate relative abundance of $\mathrm{HCl}$ of $1.5 \times 10^{-8}$, which is within an order of magnitude of our values for $\mathrm{W}$ Aql and $\chi$ Cyg.

Jorissen et al. (1992) observed a sample of AGB stars of different chemical types (and some non-AGB stars) and used mid infrared lines of $\mathrm{HF}$ as a proxy for the fluorine abundance. They find photospheric $\mathrm{F}$ abundances consistently higher in AGB stars than the solar abundance of fluorine. Abia et al. (2015) find similarly enhanced $\mathrm{F}$ abundances for their sample mostly of carbon and SC-type AGB stars, albeit to a lesser extent. The ramifications of their results will be discussed in Sect. 6.3, but the purely observational implication is that HF should be detectable for many AGB stars, if only the rotational lines were not so difficult to access from the ground.

\subsection{Chlorine abundance and isotopic ratio}

Both stable isotopes of chlorine, ${ }^{35} \mathrm{Cl}$ and ${ }^{37} \mathrm{Cl}$, are believed to be primarily formed during the hydrostatic and explosive oxygen burning stages of supernova explosions (Woosley \& Weaver 1995), through different nuclear reactions (and predominantly through core-collapse supernovae Kobayashi et al. 2020). Esteban et al. (2015) and Henry et al. (2004) found a weak trend towards decreasing $\mathrm{Cl}$ abundance with galactic radius, based on data from HII regions and planetary nebulae (PNe). They also found very similar relations between $\mathrm{Cl}$ and $\mathrm{O}$ gradients with galactic radius, suggesting that the production of $\mathrm{Cl}$ and $\mathrm{O}$ are correlated (Maas et al. 2016; Maas \& Pilachowski 2021) and hence that $\mathrm{Cl}$ can be used as a tracer of metallicity. From a study of PNe, Delgado-Inglada et al. (2015) found that $\mathrm{Cl}$ is a good indicator of metallicity in the progenitors of PNe (i.e. AGB stars). Hence, the comparison of an $\mathrm{AGB} \mathrm{Cl}$ abundance with the solar abundance might indicate a higher or lower metallicity of the natal environment of the AGB star, without obfuscation from elements actively synthesised by AGB stars (which ${ }^{35} \mathrm{Cl}$ is not, based on the investigation of Maas et al. 2016). However, the solar abundance of chlorine is very difficult to measure, for reasons discussed in detail by Maas et al. (2016). For the purposes of our study, we assume a solar abundance of $\mathrm{Cl}$, relative to $\mathrm{H}_{2}$, of $6.3 \times 10^{-7}$, based on the value given by Asplund et al. (2009), when referring to solar chlorine abundance.

The highest total chlorine abundance we find for W Aql, by summing the abundance of $\mathrm{HCl}$ and the higher abundance of $\mathrm{AlCl}$, is just over half that of the solar abundance. While we can determine from non-detections in our ALMA observations that the abundances of $\mathrm{NaCl}$ and $\mathrm{KCl}$ are less significant than those of $\mathrm{HCl}$ and $\mathrm{AlCl}$, we cannot be certain whether other molecules do not contribute to the total $\mathrm{Cl}$ abundance. For example, Agúndez et al. (2020) predict that more unusual and as yet 
undetected molecules (such as $\mathrm{CaCl}_{2}, \mathrm{SiCl}, \mathrm{ZrCl}_{2}$ ) may contribute a few percent to the overall $\mathrm{Cl}$ abundance, although they predict $\mathrm{AlCl}, \mathrm{HCl}$ and atomic $\mathrm{Cl}$ to be the dominant species for S-stars. Furthermore, their models only look at the inner regions of AGB CSEs (out to $10 R_{\star}$ ) under chemical equilibrium conditions and make no predictions for abundances outside of these regions. Hence, although we find a lower total $\mathrm{Cl}$ abundance for W Aql than solar, we cannot with certainty say that W Aql must have a lower metallicity than solar. The uncertainty of our $\mathrm{HCl}$ abundance is also significant and improved models based on spectrally resolved $\mathrm{HCl}$ lines would give rise to firmer conclusions. Although there are likely differences between carbon and S-type stars in terms of the formation and destruction of Cl-bearing molecules (see Sect. 6.1.2), we can make a firstorder approximation of the difference in total $\mathrm{Cl}$ abundance by considering the sum of abundances of the observed Cl-bearing molecules for W Aql and CW Leo (see Table 5 and Agúndez et al. 2012). Considering similar inner regions of the CSEs (where our $\mathrm{AlCl}$ and $\mathrm{HCl}$ models overlap for $\mathrm{W} \mathrm{Aql}$ ), we find a factor of two more $\mathrm{Cl}$ detected around $\mathrm{W}$ Aql than $\mathrm{CW}$ Leo. This could be because different chemical processes are in play for the different types of stars, or, if we assume the chemical processes are similar, this difference could indicate that W Aql formed from a natal cloud with higher metallicity than CW Leo.

The solar system ${ }^{35} \mathrm{Cl} /{ }^{37} \mathrm{Cl}$ ratio of 3.1 is well established (see for example Asplund et al. 2009). In close agreement with the solar value, Agúndez et al. (2011) found ${ }^{35} \mathrm{Cl} /{ }^{37} \mathrm{Cl}=3.3 \pm 0.3$ towards CW Leo from the modelling of HIFI observations of $\mathrm{HCl}$, which is also in agreement with studies of metal chlorides in the same star (e.g. $2.9 \pm 0.3$ from the Agúndez et al. 2012, study of $\mathrm{NaCl}, \mathrm{KCl}$ and $\mathrm{AlCl}$ ). Some variation has been found for this ratio in other astronomical sources, however. For example, Peng et al. (2010) observed the $(1 \rightarrow 0)$ transition of both isotopologues of $\mathrm{HCl}$ towards several different galactic sources, including star-forming regions, molecular clouds and carbon stars $^{9}$. For sources towards which both isotopologues were detected, they found a spread of ${ }^{35} \mathrm{Cl} /{ }^{37} \mathrm{Cl}$ ratios, mostly in the 2.0-2.6 range, with values below 1 for two locations in the W3 star forming region and $\sim 5$ for $\mathrm{DR} 21(\mathrm{OH})$, a region of massive star formation. Maas \& Pilachowski (2018) found ${ }^{35} \mathrm{Cl} /{ }^{37} \mathrm{Cl}$ ratios ranging from 1.76 to 3.42 for a sample of six $\mathrm{M}$ giants. All of these results point to significant variation in ${ }^{35} \mathrm{Cl} /{ }^{37} \mathrm{Cl}$ across the galaxy. The stellar evolution models of Cristallo et al. (2015) and Karakas \& Lugaro (2016) predict modest decreases in ${ }^{35} \mathrm{Cl} /{ }^{37} \mathrm{Cl}$ during the $\mathrm{AGB}$ phase, depending on metallicity and initial mass. For example, the most significant decrease in ${ }^{35} \mathrm{Cl} /{ }^{37} \mathrm{Cl}$ (to 2.17 at the end of the AGB phase) in the Karakas \& Lugaro (2016) models is seen for a low metallicity $\operatorname{star}(Z=0.007)$ with initial mass $2.75 M_{\odot}$.

The ${ }^{35} \mathrm{Cl} /{ }^{37} \mathrm{Cl}$ values we find for $\mathrm{W} \mathrm{Aql}$ from $\mathrm{AlCl}$ are 1.2 in the innermost region and 2.4 in the outer region of the $\mathrm{AlCl}$ emission. Since the $\mathrm{AlCl}$ emission is relatively faint, especially in the case of $\mathrm{Al}^{37} \mathrm{Cl}$, it is unclear to what extent the different isotopic ratios are real or a product of observational uncertainty and noise, especially since chemical fractionation is not expected to play a significant role. This uncertainty could be reduced if we had sensitive observations of additional $\mathrm{Al}^{35} \mathrm{Cl}$ and $\mathrm{Al}^{37} \mathrm{Cl}$

9 CW Leo was the only carbon star for which Peng et al. (2010) observed both isotopologues of $\mathrm{HCl}$. Their ratio is lower than that found by Agúndez et al. (2011) but still agrees within uncertainties. We consider only the Agúndez et al. (2011) value here since they use more $\mathrm{HCl}$ transitions and more detailed radiative transfer models to obtain their result. lines in the ground vibrational state, rather than just one line for each isotopologue. Alternatively, checking for a similar discrepancy in another molecule could confirm it more strongly if it were found. For example, spectrally and spatially resolved observations of $\mathrm{H}^{35} \mathrm{Cl}$ and $\mathrm{H}^{37} \mathrm{Cl}(1 \rightarrow 0)$ are possible with ALMA and could give us more information about the spatial dependence of the ${ }^{35} \mathrm{Cl} /{ }^{37} \mathrm{Cl}$ ratio. Additionally, spectrally (but not spatially) resolved observations of $\mathrm{H}^{35} \mathrm{Cl}$ and $\mathrm{H}^{37} \mathrm{Cl}$ up to $(4 \rightarrow 3)$ are possible with SOFIA $^{10}$, and would allow us to independently determine the $\mathrm{H}^{35} \mathrm{Cl}$ and $\mathrm{H}^{37} \mathrm{Cl}$ abundances and constrain our $\mathrm{HCl}$ models better than the PACS data alone.

\subsection{Abundance of fluorine}

The cosmic origin of fluorine has not yet been fully constrained, with nucleosynthesis models under-predicting observed fluorine abundances (Lugaro et al. 2004; Kobayashi et al. 2020). A significant portion of the local fluorine abundance is thought to have been produced by AGB stars, around 51\%, according to the models of Kobayashi et al. (2020). However, there are at present still several uncertainties in the calculations of nucleosynthesis yields, particularly when it comes to the treatment of convection and mass loss (Kobayashi et al. 2020). In a detailed study of the uncertainties in the nuclear reaction rates for ${ }^{19} \mathrm{~F}$, Lugaro et al. (2004) concluded that these cause uncertainties in theoretical models of fluorine production of a factor of two to seven, depending on the initial stellar mass. A recent study by Ryde et al. (2020) argues for multiple sites of fluorine production, in particular at different metallicities. Nevertheless, it has been clear for some time that AGB stars play a significant role in the production of fluorine. Jorissen et al. (1992) first determined fluorine abundances for sources outside of the solar system. From their observations of atmospheric HF, they concluded that, not only is F more abundant in M, S and carbon stars than in the Sun, it is generally further enhanced in carbon and S-type stars compared with M-type stars. Further evidence of the synthesis of F in AGB stars is provided by Zhang \& Liu (2005), who find abundances of $\mathrm{F}$ in PNe higher than the solar abundance, hence surmising that $\mathrm{F}$ is produced in the AGB progenitors of $\mathrm{PNe}$.

The solar abundance of elemental fluorine is still somewhat uncertain, not least because fluorine is the least abundant element in the range of atomic numbers from 6 to 20 (carbon to calcium, Asplund et al. 2009), and is more difficult to measure. Nevertheless, the recent solar fluorine abundance found by Maiorca et al. (2014) is in agreement within the uncertainties with earlier determinations (Lodders 2003; Asplund et al. 2009). Converting their values to abundances relative to $\mathrm{H}_{2}$ for comparison with our results, we find solar abundances of $\mathrm{F}$ in the range 5.0-7.2 $\times 10^{-8}$, with uncertainties at, or close to, a factor of two. Whichever value we adopt for the solar abundance of $\mathrm{F}$, our abundance of AlF for W Aql is higher than the solar F abundance, and increases if we include the upper limit we found for HF. This indicates that F synthesised in the AGB star has already been dredged up to the surface and injected into the wind.

As discussed in Sect. 6.1, fluorine-bearing molecules have not been extensively studied towards AGB stars, with the carbon star CW Leo being the only example of AlF and rotational lines of HF predating the present study. The total abundance of $\mathrm{F}$ found from $\mathrm{AlF}$ and $\mathrm{HF}$ for CW Leo (see Table 5) is almost an order of magnitude less than what we find for W Aql and lower than (any determination of) the solar abundance. This could be a result of different chemical processes in the winds of the two

${ }_{10}$ Stratospheric Observatory for Infrared Astronomy. 
T. Danilovich et al.: ATOMIUM: Halide molecules around the S-type AGB star W Aquilae

Table 6. Calculated rate coefficients for reactions between $\mathrm{Al}$ and halogen species (see text in Appendix D for further details).

\begin{tabular}{cl}
\hline \hline Reaction & \multicolumn{1}{c}{ Rate coefficient $^{(a)}$} \\
\hline Fluorine reactions: & \\
$\mathrm{Al}+\mathrm{HF} \rightarrow \mathrm{AlF}+\mathrm{H}$ & $k_{1}=1.1 \times 10^{-10} \exp (-125 / T)+9.3 \times 10^{-10} \exp (-2750 / T)$ \\
$\mathrm{AlOH}+\mathrm{HF} \rightarrow \mathrm{AlF}+\mathrm{H}_{2} \mathrm{O}$ & $k_{2}=7.3 \times 10^{-12} \exp (-1800 / T)+5.4 \times 10^{-12}(T / 300)^{-1.8}$ \\
$\mathrm{AlO}+\mathrm{HF} \rightarrow \mathrm{AlF}+\mathrm{OH}$ & $k_{3}=7.1 \times 10^{-10} \exp (145 / T)$ \\
$\mathrm{AlF}+\mathrm{H} \rightarrow \mathrm{Al}+\mathrm{HF}$ & $k_{-1}=1.5 \times 10^{-10} \exp (-13816 / T)$ \\
$\mathrm{AlF}+\mathrm{H}_{2} \mathrm{O} \rightarrow \mathrm{AlOH}+\mathrm{HF}$ & $k_{-2}=3.9 \times 10^{-12} \exp (-6125 / T)$ \\
$\mathrm{AlF}+\mathrm{OH} \rightarrow \mathrm{AlO}+\mathrm{HF}$ & $k_{-3}=4.1 \times 10^{-10} \exp (-4105 / T)$ \\
$\mathrm{Chlorine} \mathrm{reactions}:$ & \\
$\mathrm{Al}+\mathrm{HCl} \rightarrow \mathrm{AlCl}+\mathrm{H}$ & $k_{4}=1.4 \times 10^{-10} \exp (-890 / T)+2.0 \times 10^{-9} \exp (-4036 / T)$ \\
$\mathrm{AlOH}+\mathrm{HCl} \rightarrow \mathrm{AlCl}+\mathrm{H} \mathrm{H}_{2} \mathrm{O}$ & $k_{5}=1.9 \times 10^{-14}(T / 300)^{1.98} \exp (-630 / T)$ \\
$\mathrm{AlO}+\mathrm{HCl} \rightarrow \mathrm{AlCl}+\mathrm{OH}$ & $k_{6 a}=2.0 \times 10^{-10} \exp (-793 / T)$ \\
$\mathrm{AlCl}+\mathrm{H} \rightarrow \mathrm{AlOH}+\mathrm{Cl}+\mathrm{HCl}$ & $k_{6 b}=8.8 \times 10^{-10} \exp (-27 / T)$ \\
$\mathrm{AlCl}+\mathrm{H} \mathrm{H}_{2} \mathrm{O} \rightarrow \mathrm{AlOH}+\mathrm{HCl}$ & $k_{-5}=3.0 \times 10^{-10} \exp (-10670 / T)$ \\
$\mathrm{AlCl}+\mathrm{OH} \rightarrow \mathrm{AlO}+\mathrm{HCl}$ & $k_{-6 a}=1.7 \times 10^{-14}(T / 300)^{2.10} \exp (-3010 / T)$ \\
$\rightarrow \mathrm{AlOH}+\mathrm{Cl}$ & $k_{-6 b}=8.0 \times 10^{-10} \exp (-1266 / T)$ \\
\hline
\end{tabular}

Notes. ${ }^{(a)}$ Units for the bimolecular reaction rate coefficients are $\mathrm{cm}^{3}$ molecule ${ }^{-1} \mathrm{~s}^{-1}$.

stars, or could be explained by CW Leo having formed from a lower-metallicity natal cloud than W Aql, which would affect the initial (and hence total) F abundance. Although the chemical equilibrium predictions of Agúndez et al. (2020) predict that AlF and $\mathrm{HF}$ will be the dominant carriers of $\mathrm{F}$ in the inner winds of carbon stars as well as S-type stars (with very similar molecular abundances of F-bearing species between the two AGB types), it is possible there are some additional non-equilibrium processes at play that more strongly affect carbon stars and were not taken into account for that study. For example, the presence of $\mathrm{H}_{2} \mathrm{O}$ in the inner regions of $\mathrm{CW}$ Leo is a clear indicator of non-equilibrium processes in those regions (Decin et al. 2010).

An interesting direction of future study would be a more extensive look at AlF and HF towards a larger sample of AGB stars, especially S-type stars. As previously mentioned, AlF was detected towards some of the oxygen-rich AGB stars in the ATOMIUM sample, and will be analysed in future work. Additional observations of AlF towards S-type stars do not currently exist but could be obtained with ALMA, potentially of multiple rotational lines. Observations of $\mathrm{HF}$ are more difficult to obtain, with rotational $\mathrm{HF}$ (found in the $\mathrm{THz}$ range) not presently accessible ${ }^{11}$. Nevertheless, photospheric abundance determinations from infrared observations of HF towards AGB stars with known mass-loss rates and well-studied CSEs (including abundances of other molecules) would help fill out our understanding of $F$ towards these stars. In particular, comparing the abundances of AlF $+\mathrm{HF}$ with physical parameters, such as mass-loss rates, pulsation periods, luminosity, expansion velocity, etc, could tell us about the evolutionary history of the star, especially in light of the synthesis of $\mathrm{F}$ during the AGB phase.

\subsection{Constraints on aluminium abundance}

$\mathrm{AlO}$ and $\mathrm{AlOH}$ were not detected towards W Aql, despite being detected towards some of the oxygen-rich ATOMIUM sources (to be presented in a future study). This is notable since AlO

${ }_{11}$ At 1232.476 GHz (Nolt et al. 1987; Pickett et al. 1998), the $J=1 \rightarrow 0$ line falls close to a water line and just outside of the feasible observing ranges SOFIA is currently equipped for (Duran et al. 2021). and especially $\mathrm{AlOH}$ are expected to be the dominant carriers of Al under thermochemical equilibrium (Agúndez et al. 2020) and steady-state chemical models, even in the case of S-type stars. The absence or very low abundance (quantified below) of $\mathrm{AlO}$ and $\mathrm{AlOH}$ is indicative of other processes, such as dust formation or growth, limiting the gas-phase abundance of these molecules. In Appendix A.2, we calculate rms values as detection limits for $\mathrm{AlO}$ and $\mathrm{AlOH}$. Additionally, we ran some radiative transfer models to obtain abundance upper limits for $\mathrm{AlO}$ and $\mathrm{AlOH}$.

We tested two abundance distributions for both $\mathrm{AlO}$ and AlOH: 1) a model with a constant abundance of the molecule from the stellar surface out to $4 \times 10^{14} \mathrm{~cm}$, based partly on the results found for the oxygen-rich stars in Decin et al. (2017); and 2) a model with an abundance profile based on the predictions of the chemical model described in Sect. 4.3 and expanded on in Sect. 6.5. In both cases, the abundance was scaled until the lines predicted by the model were equal to and/or did not exceed the rms values given in Table A.1 (using the same extraction apertures). The molecular data used here is taken from Decin et al. (2017) and Danilovich et al. (2020) for the AlOH and AlO models, respectively. For the constant abundance models, we found upper limits of $f_{\mathrm{AlO}} \leq 6 \times 10^{-9}$ and $f_{\mathrm{AlOH}} \leq 3 \times 10^{-8}$, relative to $\mathrm{H}_{2}$. For the abundance distributions predicted by the chemical model, we found upper limits of $f_{\text {AlO,peak }} \leq 6.6 \times 10^{-9}$ and $f_{\text {AlOH,peak }} \leq 6.5 \times 10^{-8}$, relative to $\mathrm{H}_{2}$. Both sets of upper limits are plotted in Fig. A.1. The AlOH upper limit exceeds the abundances found for the M-type stars R Dor and IK Tau by around an order of magnitude, but the AlO upper limit is around an order of magnitude smaller than the abundances found for the same stars (Decin et al. 2017).

\subsection{Chemistry of $\mathrm{AICl}$ and AIF}

After the initial chemical model results were used as input for the radiative transfer modelling of $\mathrm{HCl}$ and $\mathrm{HF}$ (i.e. see Sect. 4.3 and the derived abundance results in Fig. 12), we extended the RATE12 chemical model by including reactions describing the chemistry of aluminium, in an attempt to reproduce the observed abundance distributions of the halide species. The aluminium 
halides studied here can be produced by the exothermic reactions of $\mathrm{Al}, \mathrm{AlOH}$ and $\mathrm{AlO}$ with the corresponding hydrogen halides:

$$
\begin{array}{lr}
\mathrm{Al}+\mathrm{HF} \rightarrow \mathrm{AlF}+\mathrm{H} & \Delta H^{\ominus}=-113 \mathrm{~kJ} \mathrm{~mol}^{-1} \\
\mathrm{AlOH}+\mathrm{HF} \rightarrow \mathrm{AlF}+\mathrm{H}_{2} \mathrm{O} & \Delta H^{\ominus}=-52 \mathrm{~kJ} \mathrm{~mol}^{-1} \\
\mathrm{AlO}+\mathrm{HF} \rightarrow \mathrm{AlF}+\mathrm{OH} & \Delta H^{\ominus}=-37 \mathrm{~kJ} \mathrm{~mol}^{-1} \\
\mathrm{Al}+\mathrm{HCl} \rightarrow \mathrm{AlCl}+\mathrm{H} & \Delta H^{\ominus}=-81 \mathrm{~kJ} \mathrm{~mol}^{-1} \\
\mathrm{AlOH}+\mathrm{HCl} \rightarrow \mathrm{AlCl}+\mathrm{H}_{2} \mathrm{O} & \Delta H^{\ominus}=-19 \mathrm{~kJ} \mathrm{~mol}^{-1} \\
\mathrm{AlO}+\mathrm{HCl} \rightarrow \mathrm{AlCl}+\mathrm{OH} & \Delta H^{\ominus}=-5 \mathrm{~kJ} \mathrm{~mol}^{-1} .
\end{array}
$$

The standard reaction enthalpies, $\Delta H^{\ominus}$, (at $0 \mathrm{~K}$ ) are calculated using the very accurate G4 method within the Gaussian suite of programs (Frisch et al. 2016). Theoretical calculations of the rate coefficients for reactions R1 to R6 (i.e. $k_{1}$ to $k_{6}$ ) and the reverse reactions R-1 to R-6 ( $k_{-1}$ to $\left.k_{-6}\right)$ are described in Appendix D and the rate coefficients are listed in Table 6 . We note that the theoretical estimate of $k_{5}$ is in good agreement with a measurement between 475 and $1275 \mathrm{~K}$ (Rogowski et al. 1989). The aluminium halides can be removed by reaction with $\mathrm{H}$ (reactions $\mathrm{R}-1$ and R-4), with $\mathrm{H}_{2} \mathrm{O}$ (R-2 and R-5) or with $\mathrm{OH}$ (R-3 and R-6). In addition, they can undergo photolysis:

$$
\begin{aligned}
& \mathrm{AlF}+\mathrm{h} v \rightarrow \mathrm{Al}+\mathrm{F} \\
& \mathrm{AlCl}+\mathrm{h} v \rightarrow \mathrm{Al}+\mathrm{Cl} .
\end{aligned}
$$

The chemical outflow model used here is based on McElroy et al. (2013) and adapted by Van de Sande et al. (2018), as described in Sect. 4.3. The complete list of reactions added to the network is given in Table D.5 and discussed in Appendix D.

The first-order rates $\left(\mathrm{s}^{-1}\right)$ for the formation and destruction of $\mathrm{AlF}$ and $\mathrm{AlCl}$ (R1 to R6, R-1 to R-6, and $J_{1}$ and $J_{2}$ ) can now be calculated as a function of radius in the outflow, using the temperature and concentrations of $\mathrm{H}_{2} \mathrm{O}, \mathrm{OH}, \mathrm{H}, \mathrm{HF}$ and $\mathrm{HCl}$ from the 1D model of W Aql (Van de Sande et al. 2018). These rates are plotted in Figs. 13a and $\mathrm{b}$ for $\mathrm{AlF}$ and $\mathrm{AlCl}$, respectively, along with the molecular expansion rate (expressed as $2 v_{\infty} / R$, where $v_{\infty}=16.5 \mathrm{~km} \mathrm{~s}^{-1}$ ). This shows that the production and loss rates of both $\mathrm{AlF}$ and $\mathrm{AlCl}$ are slower than the expansion rate out to $2 \times 10^{16} \mathrm{~cm}$. In the case of $\mathrm{AlF}$, Fig. 13a shows that $\mathrm{AlF}$ is mostly produced in the inner region of the model by the reaction of $\mathrm{Al}$ with $\mathrm{HF}(\mathrm{R} 1)$, although production by $\mathrm{AlO}+\mathrm{HF}$ (R3) becomes more important beyond $2 \times 10^{15} \mathrm{~cm}$. Removal of AlF by reaction with $\mathrm{H}_{2} \mathrm{O}(\mathrm{R}-2)$ is most important in the inner region between $2 \times 10^{14}$ and $1.5 \times 10^{15} \mathrm{~cm}$, because $\mathrm{R}-1$ has a substantial activation energy so that reactions with $\mathrm{H}$ are not a competitive loss term for AlF, and the abundance of $\mathrm{OH}$ is relatively low. At distances $>2 \times 10^{15} \mathrm{~cm}$, photolysis by interstellar radiation becomes the dominant removal process.

The newly modelled concentration profiles of the halogen species are shown as a function of radius in Fig. 14, plotted with the results of the radiative transfer models (Sect. 5). The model successfully simulates the observed absolute relative abundance of AlF at radii $<3 \times 10^{15} \mathrm{~cm}$ (cf. Fig. 12). However, the AlF photolysis rate does not start to approach the expansion rate until a radial distance of $5 \times 10^{16} \mathrm{~cm}$. Hence, AlF is nearly unchanged until $>10^{16} \mathrm{~cm}$ (Fig. 14), in comparison with the observed disappearance of AlF around $3 \times 10^{15} \mathrm{~cm}$ (Fig. 12). This discrepancy probably indicates that $\mathrm{AlF}$ is efficiently removed in the cooler part of the outflow $\left(>10^{15} \mathrm{~cm}\right.$, where the temperature is below $400 \mathrm{~K}$ ) by clustering with other metallic molecules such as oxides (e.g. $\mathrm{FeO}$ and $\mathrm{MgO}$ ) and hydroxides $(\mathrm{FeOH}$ and $\mathrm{MgOH})$, as well as small dust particles (e.g. $\left(\mathrm{Al}_{2} \mathrm{O}_{3}\right)_{n},\left(\mathrm{FeMgSiO}_{4}\right)_{n}$,
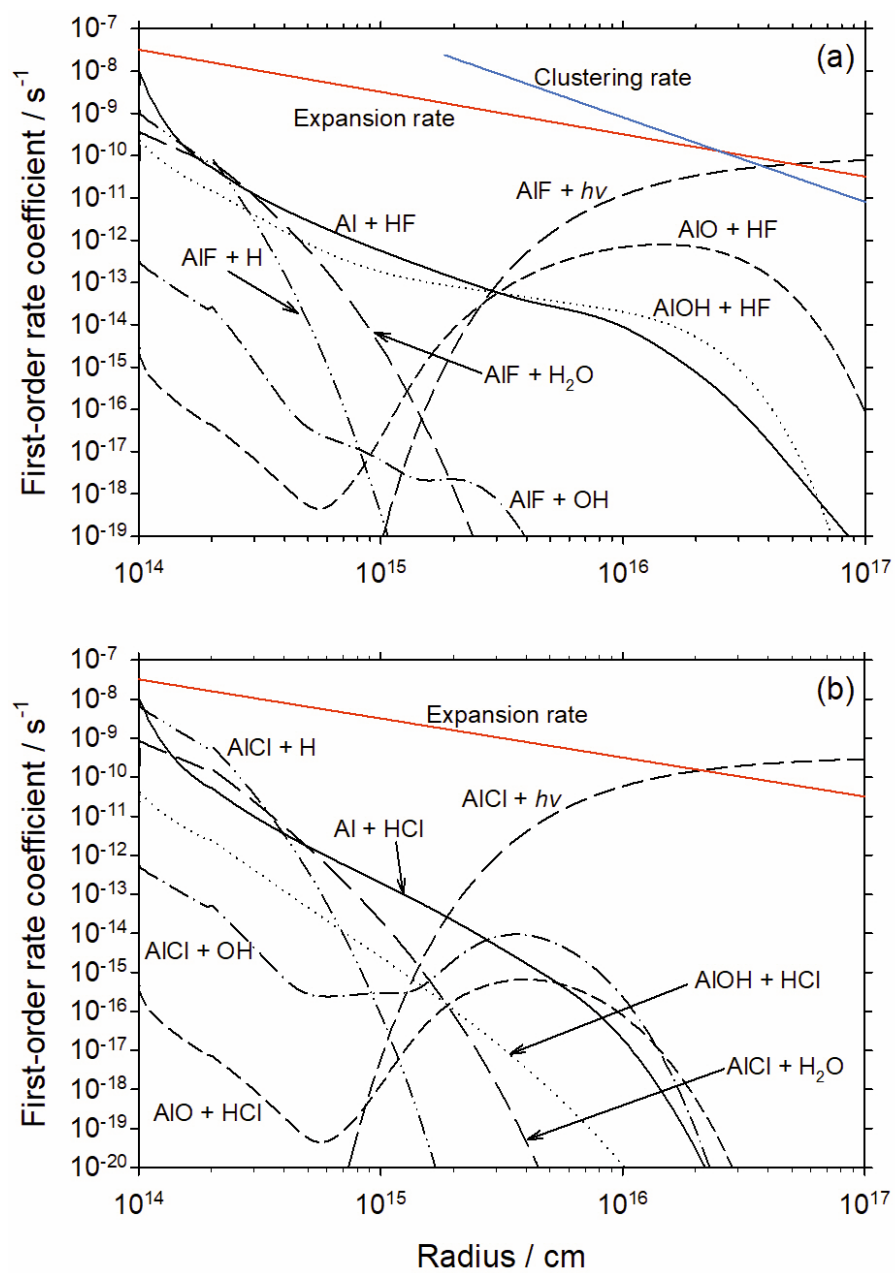

Fig. 13. (a) Calculated first-order rates for conversion of HF to AlF by reaction with $\mathrm{Al}, \mathrm{AlOH}$ and $\mathrm{AlO}$; and loss of $\mathrm{AlF}$ by photolysis and reaction with $\mathrm{H}, \mathrm{H}_{2} \mathrm{O}$ and $\mathrm{OH}$. The clustering rate with metallic compounds (blue line, see text for further details) is shown in the region where the temperature is below $500 \mathrm{~K}$. (b) Calculated first-order rates for conversion of $\mathrm{HCl}$ to $\mathrm{AlCl}$ by reaction with $\mathrm{Al}, \mathrm{AlOH}$ and $\mathrm{AlO}$; and loss of $\mathrm{AlCl}$ by photolysis and reaction with $\mathrm{H}, \mathrm{H}_{2} \mathrm{O}$ and $\mathrm{OH}$. The red lines show the molecular expansion rate of the outflow at a constant velocity of $16.5 \mathrm{~km} \mathrm{~s}^{-1}$.

$n>1)$. The total concentration of the major metals ( $\mathrm{Mg}, \mathrm{Fe}, \mathrm{Al}$ and $\mathrm{Na}$ ) relative to $\mathrm{H}_{2}$ is $7.6 \times 10^{-5}$ (Asplund et al. 2009). Clustering is likely to be fast because metal-containing molecules have large dipole moments. Assuming a clustering rate coefficient of $5 \times 10^{-10} \mathrm{~cm}^{3}$ molecule ${ }^{-1} \mathrm{~s}^{-1}$ (i.e. a typical dipole-dipole capture frequency Saunders \& Plane 2006), then the blue line in Fig. 13(a) shows that the first-order clustering rate in this cooler region is faster than the expansion rate out to $2 \times 10^{16} \mathrm{~cm}$. This means that the observed disappearance of AlF by $4 \times 10^{15} \mathrm{~cm}$ (Fig. 12) could be explained by cluster formation or uptake on dust particles.

In the case of $\mathrm{AlCl}$, the production and loss rates as a function of distance are illustrated in Fig. 13b. The reaction of $\mathrm{Al}$ with $\mathrm{HCl}(\mathrm{R} 4)$ is the most important $\mathrm{AlCl}$ production term over the entire outflow, and photolysis dominates $\mathrm{AlCl}$ removal beyond $2 \times 10^{14} \mathrm{~cm}$. The newly simulated $\mathrm{HCl}$ density (Fig. 14) is in good accord with observations, including its disappearance beyond $10^{16} \mathrm{~cm}$. However, the location of the drop-off in $\mathrm{HCl}$ abundance is not tightly constrained by our current radiative transfer model, in the absence of higher quality data (see 

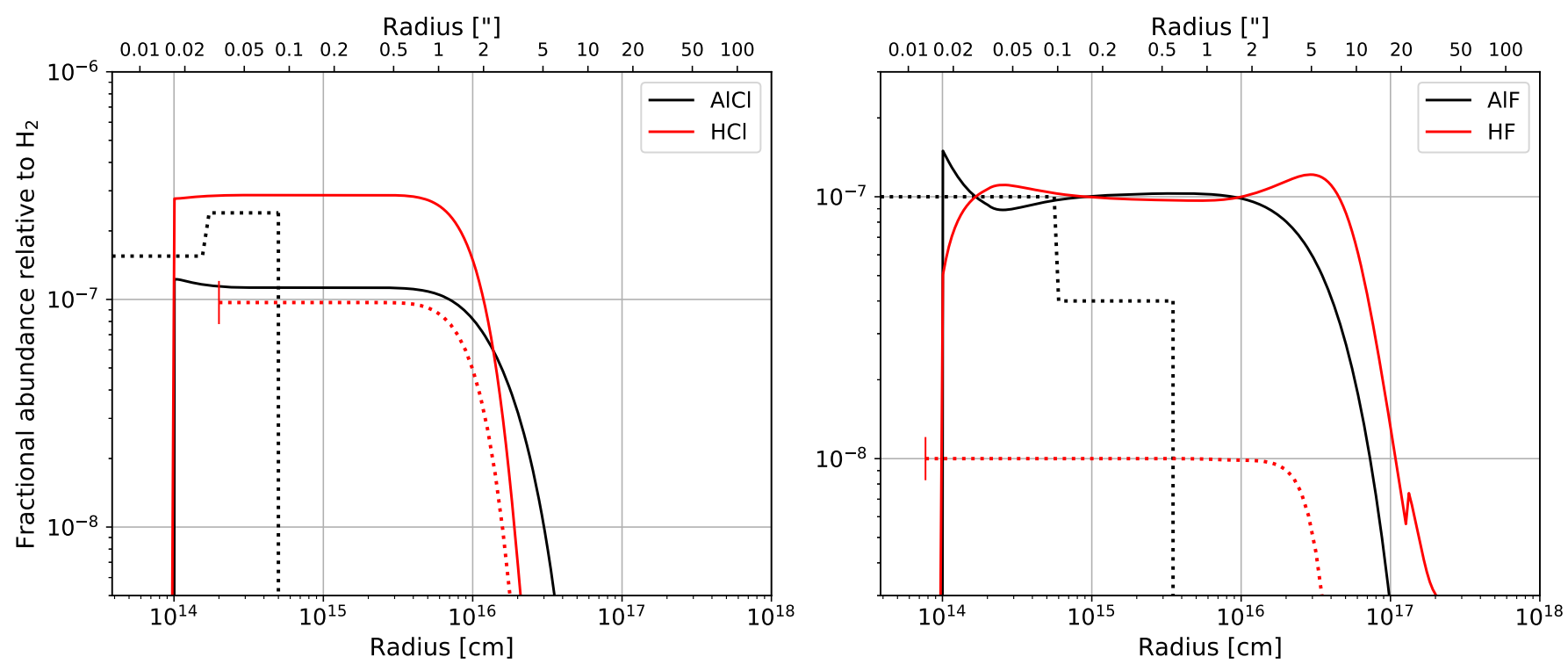

Fig. 14. Fractional abundance relative to $\mathrm{H}_{2}$ of $\mathrm{AlCl}$ and $\mathrm{HCl}$ (left panel), and $\mathrm{AlF}$ and $\mathrm{HF}$ (right panel), obtained from chemical modelling (solid lines), plotted with the results of the radiative transfer models (dotted lines). The assumed physical conditions and parent species are given in the text and Appendix D.

Sect. 5.3.1). Although the model simulates well the absolute $\mathrm{AlCl}$ density out to $5 \times 10^{14} \mathrm{~cm}$, it fails to reproduce the rapid decrease in $\mathrm{AlCl}$ further out. The reason(s) for this are unclear. Removal of $\mathrm{AlCl}$ on dust or molecular clustering might be an explanation, but this would require that $\mathrm{AlCl}$ was removed much more efficiently than AlF, which seems unlikely because $\mathrm{AlF}$ is much less volatile than $\mathrm{AlCl}$. For example, the heat of vaporisation of $\mathrm{AlF}$ from $\mathrm{AlF}_{3}$ is $1227 \mathrm{~kJ} \mathrm{~mol}^{-1}$ at $1000 \mathrm{~K}$, whereas that of $\mathrm{AlCl}$ from $\mathrm{AlCl}_{3}$ is only $620 \mathrm{~kJ} \mathrm{~mol}^{-1}$ (Chase et al. 1985). So this remains an interesting challenge for future study.

\section{Conclusions}

We presented observations of $\mathrm{AlCl}$ and $\mathrm{AlF}$ towards an S-type AGB star for the first time. We detected rotational lines of $\mathrm{AlCl}$ up to the second vibrationally excited state and one rotational line of $\mathrm{AlF}$ in the ground vibrational state. $\mathrm{AlCl}$ was found in regions very close to the star, within $0 .{ }^{\prime} 1$, while AlF was found in a larger region of the envelope, out to $0.2-0 .{ }^{\prime} 6$. The distribution of both molecules was slightly asymmetric, probably due to regions of higher and lower density around the star.

The observations of $\mathrm{AlCl}$ and $\mathrm{AlF}$ were azimuthally averaged and analysed using 1D non-LTE radiative transfer models. We found step-function abundance profiles best reproduce the ALMA observations, with $\mathrm{Al}^{35} \mathrm{Cl}$ increasing at $\sim 3.6 R_{\star}$ from an abundance of $8.5 \times 10^{-8}$ to $1.7 \times 10^{-7}$, relative to $\mathrm{H}_{2}$, and no longer present from $\sim 13 R_{\star}$. For $\mathrm{Al}^{37} \mathrm{Cl}$ we did not find a step-function in abundance (possibly due to the fainter data) and instead found a constant abundance of $7 \times 10^{-8}$ relative to $\mathrm{H}_{2}$, also out to $\sim 13 R_{\star}$. For AlF we found a higher abundance in the inner region close to the star, with abundance $1 \times 10^{-7}$ relative to $\mathrm{H}_{2}$ out to $\sim 16 R_{\star}$, after which it dropped down to $4 \times 10^{-8}$ until $\sim 90 R_{\star}$, beyond which it was not detected. The $\mathrm{AlCl}$ and $\mathrm{AlF}$ abundances found for W Aql are higher than those seen for the carbon star CW Leo, and distributed differently in the CSE. This points to different chemical processes taking part in the creation and destruction of these molecules in the S-type W Aql and the carbon-rich CW Leo.
In addition to the ALMA observations of $\mathrm{AlCl}$ and $\mathrm{AlF}$, we used radiative transfer models and unresolved PACS spectra of $\mathrm{HCl}$ and $\mathrm{HF}$ towards W Aql to constrain the abundances of those molecules, using predictions from chemical models to determine the size of the corresponding molecular envelopes. We found an $\mathrm{HCl}$ abundance of $9.7 \times 10^{-8}$, relative to $\mathrm{H}_{2}$, and were able to put an upper limit on $\mathrm{HF}$ of $\leq 1 \times 10^{-8}$. We also modelled $\mathrm{HCl}$ and HF for another S-type AGB star: $\chi$ Cyg and found a slightly lower abundance of $\mathrm{HCl}\left(6.5 \times 10^{-8}\right.$ relative to $\left.\mathrm{H}_{2}\right)$ and a higher abundance of HF $\left(1.2 \times 10^{-8}\right)$.

The total abundance of $\mathrm{F}$ calculated for $\mathrm{W}$ Aql (even if we exclude the upper limit found for HF) is higher than the solar abundance of $\mathrm{F}$. This indicates that not only has $\mathrm{F}$ been synthesised in W Aql, as is expected for AGB stars, but it has also been dredged up to the surface and ejected into the CSE.

From an analysis of chemical reactions in the wind, we find that gas-phase reactions alone cannot explain the abundance distributions of $\mathrm{AlCl}$ and $\mathrm{AlF}$ found from the observations and radiative transfer modelling. We conclude that $\mathrm{AlF}$ is most likely removed from the gas phase due to clustering (i.e. as part of the dust formation process). However, the very rapid removal of $\mathrm{AlCl}$ may be due to an additional factor that cannot yet be fully explained.

Acknowledgements. We thank the anonymous referee for their thoughtful feedback on the manuscript. T.D., M.V.dS. and S.H.J.W. acknowledge support from the Research Foundation Flanders (FWO) through grants 12N9920N, $12 \mathrm{X} 6419 \mathrm{~N}$, and $1285221 \mathrm{~N}$, respectively. T.J.M. is grateful to the STFC for support through grant ST/P000312/1. J.M.C.P. was supported by STFC grant number ST/T000287/1. L.D., J.M.C.P., W.H., S.H.J.W., D.G. acknowledge support from ERC consolidator grant 646758 AEROSOL. E.D.B. acknowledges support from the Swedish National Space Agency. E.C. acknowledges funding from the KU Leuven C1 grant MAESTRO C16/17/007. This paper makes use of the following ALMA data: ADS/JAO.ALMA\#2018.1.00659.L. ALMA is a partnership of ESO (representing its member states), NSF (USA) and NINS (Japan), together with NRC (Canada), MOST and ASIAA (Taiwan), and KASI (Republic of Korea), in cooperation with the Republic of Chile. The Joint ALMA Observatory is operated by ESO, AUI/NRAO and NAOJ. We acknowledge excellent support from the UK ALMA Regional Centre (UK ARC), which is hosted by the Jodrell Bank Centre for Astrophysics (JBCA) at the University of Manchester. The UK ARC Node is supported by STFC Grant ST/P000827/1. PACS has been developed by a consortium of institutes led by MPE 
(Germany) and including UVIE (Austria); KU Leuven, CSL, IMEC (Belgium); CEA, LAM (France); MPIA (Germany); INAF-IFSI/OAA/OAP/OAT, LENS, SISSA (Italy); IAC (Spain). This development has been supported by the funding agencies BMVIT (Austria), ESA-PRODEX (Belgium), CEA/CNES (France), DLR (Germany), ASI/INAF (Italy), and CICYT/MCYT (Spain). This work has made use of data from the European Space Agency (ESA) mission Gaia (https://www. cosmos. esa.int/gaia), processed by the Gaia Data Processing and Analysis Consortium (DPAC, https://www. cosmos.esa.int/web/ gaia/dpac/consortium). Funding for the DPAC has been provided by national institutions, in particular the institutions participating in the Gaia Multilateral Agreement.

\section{References}

Abia, C., Cunha, K., Cristallo, S., \& de Laverny, P. 2015, A\&A, 581, A88 Agúndez, M., Cernicharo, J., Waters, L. B. F. M., et al. 2011, A\&A, 533, L6 Agúndez, M., Fonfría, J. P., Cernicharo, J., et al. 2012, A\&A, 543, A48

Agúndez, M., Martínez, J. I., de Andres, P. L., Cernicharo, J., \& Martín-Gago, J. A. 2020, A\&A, 637, A59

Apponi, A. J., Barclay, W. L., J., \& Ziurys, L. M. 1993, ApJ, 414, L129

Arthurs, A. M., \& Dalgarno, A. 1960, Proc. R. Soc. London Ser. A, 540 Asplund, M., Grevesse, N., Sauval, A. J., \& Scott, P. 2009, ARA\&A, 47, 481

Atkinson, R., Baulch, D. L., Cox, R. A., et al. 2006, Atmos. Chem. Phys., 6, 3625

Barton, E. J., Chiu, C., Golpayegani, S., et al. 2014, MNRAS, 442, 1821

Bauernschmitt, R., \& Ahlrichs, R. 1996, Chem. Phys. Lett., 256, 454

Brunner, M., Danilovich, T., Ramstedt, S., et al. 2018, A\&A, 617, A23

Cabezas, C., Cernicharo, J., Quintana-Lacaci, G., et al. 2016, ApJ, 825, 150

Cardelli, J. A., Clayton, G. C., \& Mathis, J. S. 1989, ApJ, 345, 245

Caris, M., Lewen, F., \& Winnewisser, G. 2002, Z. Naturf. Teil A, 57, 663

Caris, M., Lewen, F., Müller, H. S. P., \& Winnewisser, G. 2004, J. Mol. Struct., 695,243

Cernicharo, J., \& Guelin, M. 1987, A\&A, 183, L10

Cernicharo, J., Decin, L., Barlow, M. J., et al. 2010, A\&A, 518, L136

Chase, M. W., Davies, C. A., Downey, J. R., et al. 1985, NIST-JANAF Thermochemical Tables 1985 Version 1.0 (Gaithersburg, MD: National Institute of Standards and Technology Gaithersburg)

Cherchneff, I. 2012, A\&A, 545, A12

Clouser, P. L., \& Gordy, W. 1964, Phys. Rev., 134, A863

Cristallo, S., Straniero, O., Piersanti, L., \& Gobrecht, D. 2015, ApJS, 219, 40

Curtiss, L. A., Redfern, P. C., \& Raghavachari, K. 2007, J. Chem. Phys., 126, 084108

Danilovich, T., Bergman, P., Justtanont, K., et al. 2014, A\&A, 569, A76

Danilovich, T., Olofsson, G., Black, J. H., Justtanont, K., \& Olofsson, H. 2015a A\&A, 574, A23

Danilovich, T., Teyssier, D., Justtanont, K., et al. 2015b, A\&A, 581, A60

Danilovich, T., Richards, A. M. S., Karakas, A. I., et al. 2019, MNRAS, 484, 494

Danilovich, T., Gottlieb, C. A., Decin, L., et al. 2020, ApJ, 904, 110

De Beck, E., \& Olofsson, H. 2018, A\&A, 615, A8

De Beck, E., \& Olofsson, H. 2020, A\&A, 642, A20

de Graauw, T., Helmich, F. P., Phillips, T. G., et al. 2010, A\&A, 518, L6

Decin, L., Agúndez, M., Barlow, M. J., et al. 2010, Nature, 467, 64

Decin, L., Richards, A. M. S., Waters, L. B. F. M., et al. 2017, A\&A, 608, A55

Decin, L., Richards, A. M. S., Danilovich, T., Homan, W., \& Nuth, J. A. 2018 , A\&A, 615, A28

Decin, L., Montargès, M., Richards, A. M. S., et al. 2020, Science, 369, 1497

Delgado-Inglada, G., Rodríguez, M., Peimbert, M., Stasińska, G., \& Morisset, C. 2015, MNRAS, 449, 1797

Doan, L., Ramstedt, S., Vlemmings, W. H. T., et al. 2017, A\&A, 605, A28

Dubernet, M.-L., Alexander, M. H., Ba, Y. A., et al. 2013, A\&A, 553, A50

Duran, C. A., Gusten, R., Risacher, C., et al. 2021, IEEE Trans. Terahertz Sci. Technol., 11, 194

Esteban, C., García-Rojas, J., \& Pérez-Mesa, V. 2015, MNRAS, 452, 1553

Frisch, M. J., Trucks, G. W., Schlegel, H. B., et al. 2016, Gaussian 16 Revision B.01, (Wallingford, CT: Gaussian Inc.)

Gaia Collaboration (Prusti, T., et al.) 2016, A\&A, 595, A1

Gaia Collaboration (Brown, A. G. A., et al.) 2021, A\&A, 649, A1

Gao, Y., Alecu, I. M., Hsieh, P.-C., et al. 2006, J. Phys. Chem. A, 110, 6844

Georgievskii, Y., \& Klippenstein, S. J. 2005, J. Chem. Phys., 122, 194103

Gilbert, R. G., \& Smith, S. C. 1990, Theory of Unimolecular and Recombination Reactions (Oxford, Boston, Brookline Village, Mass.: Blackwell Scientific Publications Publishers' Business Services Distributor)

Glowacki, D. R., Liang, C.-H., Morley, C., Pilling, M. J., \& Robertson, S. H. 2012, J. Phys. Chem. A, 116, 9545

Gobrecht, D., Cherchneff, I., Sarangi, A., Plane, J. M. C., \& Bromley, S. T. 2016, A\&A, 585, A6

Goddon, D., Groh, A., Hanses, H. J., Schneider, M., \& Urban, W. 1991, J. Mol. Spectr., 147, 392
González Delgado, D., Olofsson, H., Kerschbaum, F., et al. 2003, A\&A, 411, 123 Gordon, I., Rothman, L., Hill, C., et al. 2017, J. Quant. Spectr. Rad. Transfer (HITRAN2016 Special Issue), 203, 3

Gotoum, N., Hammami, K., Owono Owono, L. C., \& Jaidane, N.-E. 2012, Ap\&SS, 337, 553

Gottlieb, C. A., Decin, L., Richards, A. M. S., et al. 2021, A\&A, submitted

Gray, R., Corbally, C., \& Burgasser, A. 2009, Stellar Spectral Classification, Princeton Series in Astrophysics (Princeton University Press)

Groenewegen, M. A. T., Waelkens, C., Barlow, M. J., et al. 2011, A\&A, 526, A162

Guillon, G., \& Stoecklin, T. 2012, MNRAS, 420, 579

Hammami, K., Nkem, C., Owono Owono, L. C., Jaidane, N., \& Ben Lakhdar Z. 2008, J. Chem. Phys., 129, 204305

Heays, A. N., Bosman, A. D., \& van Dishoeck, E. F. 2017, A\&A, 602, A105

Henry, R. B. C., Kwitter, K. B., \& Balick, B. 2004, ApJ, 127, 2284

Hensel, K. D., Styger, C., Jäger, W., Merer, A. J., \& Gerry, M. C. L. 1993, J. Chem. Phys., 99, 3320

Herwig, F. 2005, ARA\&A, 43, 435

Hoeft, J., Lovas, F. J., Tiemann, E., \& Törring, T. 1969, Z. Naturforsch. A, 24, 1422

Hoeft, J., Lovas, F. J., Tiemann, E., \& Törring, T. 1970, Z. Naturforsch. A, 25, 1029

Höfner, S., \& Olofsson, H. 2018, A\&ARv, 26, 1

Homan, W., Montargès, M., Pimpanuwat, B., et al. 2020, A\&A, 644, A61

Hony, S., Heras, A. M., Molster, F. J., \& Smolders, K. 2009, A\&A, 501, 609

Hutson, J. M., \& Green, S. 2012, MOLSCAT: MOLecular SCATtering v. 14

Jorissen, A., Smith, V. V., \& Lambert, D. L. 1992, A\&A, 261, 164

Justtanont, K., Muller, S., Barlow, M. J., et al. 2019, in IAU Symposium, 343, eds. F. Kerschbaum, M. Groenewegen, \& H. Olofsson, 436

Karakas, A. I., \& Lugaro, M. 2016, ApJ, 825, 26

Khouri, T., Vlemmings, W. H. T., Ramstedt, S., et al. 2016, MNRAS, 463, L74

Kłos, J., \& Lique, F. 2008, MNRAS, 390, 239

Kobayashi, C., Karakas, A. I., \& Lugaro, M. 2020, ApJ, 900, 179

Kumaran, S. S., Lim, K. P., \& Michael, J. V. 1994, J. Chem. Phys., 101, 9487

Lanza, M., Kalugina, Y., Wiesenfeld, L., \& Lique, F. 2014, J. Chem. Phys., 140, 064316

Lindegren, L., Bastian, U., Biermann, M., et al. 2021, A\&A, 649, A4

Lodders, K. 2003, ApJ, 591, 1220

Lugaro, M., Ugalde, C., Karakas, A. I., et al. 2004, ApJ, 615, 934

Maas, Z. G., \& Pilachowski, C. A. 2018, AJ, 156, 2

Maas, Z. G., \& Pilachowski, C. A. 2021, AJ, 161, 183

Maas, Z. G., Pilachowski, C. A., \& Hinkle, K. 2016, AJ, 152, 196

Maiorca, E., Uitenbroek, H., Uttenthaler, S., et al. 2014, ApJ, 788, 149

Mangan, T. P., Douglas, K. M., Lade, R. E., et al. 2021, ACS Earth Space Chem., submitted

Mayer, A., Jorissen, A., Kerschbaum, F., et al. 2013, A\&A, 549, A69

McElroy, D., Walsh, C., Markwick, A. J., et al. 2013, A\&A, 550, A36

McGuire, P., \& Kouri, D. J. 1974, J. Chem. Phys., 60, 2488

Milam, S. N., Apponi, A. J., Woolf, N. J., \& Ziurys, L. M. 2007, ApJ, 668, L131

Müller, H. S. P., Thorwirth, S., Roth, D. A., \& Winnewisser, G. 2001, A\&A, 370 L49

Müller, H. S. P., Schlöder, F., Stutzki, J., \& Winnewisser, G. 2005, J. Mol. Struct., 742,215

Murty, A. N., \& Curl, R. F. 1969, J. Mol. Spectr., 30, 102

Naouai, M., Najar, F., \& Hammami, K. 2019, Phys. Chem. Chem. Phys., 21, 20137

Nicolaes, D., Groenewegen, M. A. T., Royer, P., et al. 2018, A\&A, 618, A143

Nolt, I., Radostitz, J., DiLonardo, G., et al. 1987, J. Mol. Spectr., 125, 274

Pamboundom, M., Tchakoua, T., \& Nsangou, M. 2016, Ap\&SS, 361, 150

Peng, R., Yoshida, H., Chamberlin, R. A., et al. 2010, ApJ, 723, 218

Pickett, H. M., Poynter, R. L., Cohen, E. A., et al. 1998, J. Quant. Spectr. Rad. Transf., 60, 883

Pilbratt, G. L., Riedinger, J. R., Passvogel, T., et al. 2010, A\&A, 518, L1

Pine, A. S., Fried, A., \& Elkins, J. W. 1985, J. Mol. Spectr., 109, 30

Plane, J. M. C., Daly, S. M., Feng, W., Gerding, M., \& Gómez Martín, J. C. 2021, J. Geophys. Res.: Space Phys., 126, e28792

Poglitsch, A., Waelkens, C., Geis, N., et al. 2010, A\&A, 518, L2

Quintana-Lacaci, G., Cernicharo, J., Agúndez, M., et al. 2016, ApJ, 818, 192

Ramstedt, S., Schöier, F. L., \& Olofsson, H. 2009, A\&A, 499, 515

Ramstedt, S., Maercker, M., Olofsson, G., Olofsson, H., \& Schöier, F. L. 2011, A\&A, 531, A148

Ramstedt, S., Mohamed, S., Vlemmings, W. H. T., et al. 2017, A\&A, 605, A126

Rogowski, D. F., Marshall, P., \& Fontijn, A. 1989, J. Phys. Chem., 93, 1118

Rybicki, G. B., \& Hummer, D. G. 1991, A\&A, 245, 171

Ryde, N., Jönsson, H., Mace, G., et al. 2020, ApJ, 893, 37

Saunders, R., \& Plane, J. 2006, J. Atm. Sol.-Terr. Phys., 68, 2182 
Schöier, F. L., \& Olofsson, H. 2001, A\&A, 368, 969

Schöier, F. L., van der Tak, F. F. S., van Dishoeck, E. F., \& Black, J. H. 2005, A\&A, 432, 369

Schöier, F. L., Maercker, M., Justtanont, K., et al. 2011, A\&A, 530, A83

Smolders, K., Neyskens, P., Blommaert, J. A. D. L., et al. 2012, A\&A, 540, A72

Stevens, P. S., Brune, W. H., \& Anderson, J. G. 1989, J. Phys. Chem., 93, 4068

Suh, K.-W. 2000, MNRAS, 315, 740

Tennyson, J., Yurchenko, S. N., Al-Refaie, A. F., et al. 2016, J. Mol. Spectr., 327, 73

Timp, B. A., Doran, J. L., Iyer, S., Grabow, J.-U., \& Leopold, K. R. 2012, J. Mol. Spectr., 271, 20

Tizniti, M., Le Picard, S. D., Lique, F., et al. 2014, Nat. Chem., 6, 141

Törring, T., \& Herrmann, R. 1989, Mol. Phys., 68, 1379

Turnshek, D. E., Turnshek, D. A., \& Craine, E. R. 1985, An atlas of digital spectra of cool stars

Uehara, H., Horiai, K., Nakagawa, K., \& Fujimoto, T. 1989, J. Mol. Spectr., 134, 98

Van de Sande, M., Sundqvist, J. O., Millar, T. J., et al. 2018, A\&A, 616, A106

van der Tak, F. F. S., Lique, F., Faure, A., Black, J. H., \& van Dishoeck, E. F. 2020, Atoms, 8, 15

Velilla Prieto, L., Sánchez Contreras, C., Cernicharo, J., et al. 2017, A\&A, 597, A25

Woosley, S. E., \& Weaver, T. A. 1995, ApJS, 101, 181

Wyse, F. C., \& Gordy, W. 1972, J. Chem. Phys., 56, 2130

Wyse, F. C., Gordy, W., \& Pearson, E. F. 1970, J. Chem. Phys., 52, 3887

Yamada, C., Cohen, E. A., Fujitake, M., \& Hirota, E. 1990, J. Chem. Phys., 92, 2146

Yamamura, I., Kawaguchi, K., \& Ridgway, S. T. 2000, ApJ, 528, L33

Yousefi, M., \& Bernath, P. F. 2018, ApJS, 237, 8

Zhang, Y., \& Liu, X. W. 2005, ApJ, 631, L6

1 Department of Physics and Astronomy, Institute of Astronomy, KU Leuven, Celestijnenlaan 200D, 3001 Leuven, Belgium e-mail: taissa.danilovich@kuleuven. be

${ }^{2}$ University of Leeds, School of Chemistry, Leeds LS2 9JT, UK

3 Astrophysics Research Centre, School of Mathematics and Physics, Queen's University Belfast, University Road, Belfast BT7 1NN, UK
${ }^{4}$ LSAMA, Department of Physics, Faculty of Sciences, University Tunis El Manar, Campus Universitaire, 1060 Tunis, Tunisia

${ }^{5}$ Harvard-Smithsonian Center for Astrophysics, 60 Garden Street, Cambridge, MA 02138, USA

6 JBCA, Department Physics and Astronomy, University of Manchester, Manchester M13 9PL, UK

7 Department of Space, Earth and Environment, Chalmers University of Technology, Onsala Space Observatory, 43992 Onsala, Sweden

8 Université de Bordeaux, Laboratoire d'Astrophysique de Bordeaux, 33615 Pessac, France

9 University of Amsterdam, Anton Pannekoek Institute for Astronomy, 1090 GE Amsterdam, The Netherlands

${ }^{10}$ National Astronomical Research Institute of Thailand, Chiangmai 50180, Thailand

11 Institut d'Astronomie et d'Astrophysique, Université Libre de Bruxelles (ULB), CP 226, 1060 Brussels, Belgium

12 Max-Planck-Institut für Radioastronomie, 53121 Bonn, Germany

${ }^{13}$ LESIA, Observatoire de Paris, Université PSL, CNRS, Sorbonne Université, Université de Paris, 5 place Jules Janssen, 92195 Meudon, France

${ }^{14}$ Université Côte d'Azur, Laboratoire Lagrange, Observatoire de la Côte d'Azur, 06304 Nice Cedex 4, France

${ }^{15}$ Universität zu Köln, I. Physikalisches Institut, 50937 Köln, Germany

${ }^{16}$ California Institute of Technology, Jet Propulsion Laboratory, Pasadena, CA 91109, USA

17 SRON Netherlands Institute for Space Research, 3584 CA Utrecht, The Netherlands

18 Radboud University, Institute for Mathematics, Astrophysics and Particle Physics (IMAPP), Nijmegen, The Netherlands

${ }^{19}$ Institut de Radioastronomie Millimétrique, 300 rue de la Piscine, 38406 Saint Martin d'Hères, France

${ }^{20}$ University College London, Department of Physics and Astronomy, London WC1E 6BT, UK

${ }^{21}$ University of Hong Kong, Laboratory for Space Research, Pokfulam, Hong Kong

${ }^{22}$ School of Physical Sciences, The Open University, Walton Hall, Milton Keynes, MK7 6AA, UK 


\section{Appendix A: Non-detections of other halogen- and aluminium-bearing molecules}

Appendix A.1: $\mathrm{NaCl}$ and $\mathrm{KCl}$

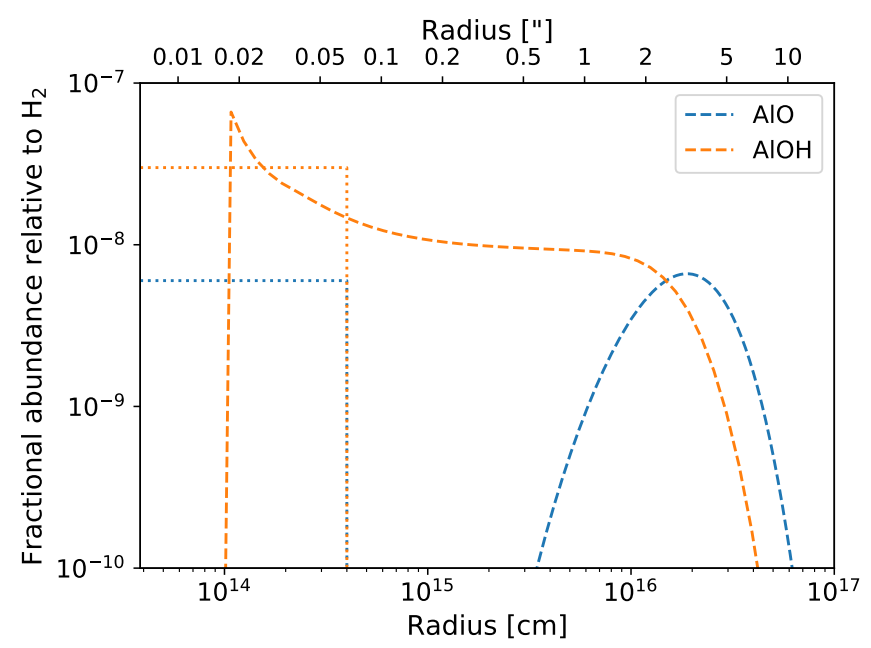

Fig. A.1. Upper limits for $\mathrm{AlO}$ (blue) and $\mathrm{AlOH}$ (orange) abundance distributions. Dotted lines show the constant abundance models and dashed lines show abundance distributions predicted by chemical models. See text for details.

$\mathrm{No} \mathrm{NaCl}$ or $\mathrm{KCl}$ lines were detected towards $\mathrm{W}$ Aql in the ATOMIUM survey. To give an indication of the detection limits of our data, we measured the rms values of the spectra near the vibrational ground state $\mathrm{NaCl}$ and $\mathrm{KCl}$ lines covered by ATOMIUM. For each line listed in Table A.1 we calculated the rms over a velocity range of $100 \mathrm{~km} \mathrm{~s}^{-1}$, centred on the line frequency. The calculation was done for the spectrum extracted from the extended array with a 0.11 radius aperture and for the spectrum from the mid array extracted with a $00^{\prime} 6$ radius aperture. These were chosen because we do not know a priori the extent of the possible $\mathrm{NaCl}$ or $\mathrm{KCl}$ lines and by checking for compact and more extended emission we can be sure of the non-detection of the salt lines. Where a line fell close to the edge of a band or close to a detected line, we measured the rms only for the unaffected half of the spectrum (over a velocity range of $50 \mathrm{~km} \mathrm{~s}^{-1}$ ). The $\mathrm{NaCl}(20 \rightarrow 19)$ line at $260.223 \mathrm{GHz}$ was excluded from the measurement since it is dominated by an overlap with a $v_{2}=1$ $\mathrm{H}^{13} \mathrm{CN}$ line. The rms values are given in Table A.1.

\section{Appendix A.2: $\mathrm{AlO}$ and $\mathrm{AlOH}$}

We performed similar measurements for $\mathrm{AlO}$ and $\mathrm{AlOH}$, with the rms values also listed in Table A.1. For these two molecules we additionally ran some radiative transfer models to ascertain the upper limits on their abundances. The results for two sets of models are shown in Fig. A.1. This was done for the aluminiumbearing molecules because they are expected to play a key role in circumstellar chemistry (see Sect. 6.5).

\section{Appendix B: Molecular data and collisional rates}

\section{Appendix B.1: AlCl}

For both $\mathrm{AlCl}$ isotopologues, we included molecular data for levels with $J \leq 40$ and $v \leq 10$. The maximum vibrationally excited level of $v=10$ was chosen because the term energy of this level is $2.2 \mu \mathrm{m}$, close to the wavelength of peak flux for W Aql, as seen from its SED (Danilovich et al. 2014). The radiative information used in our $\mathrm{AlCl}$ models comes from Yousefi \& Bernath (2018), accessed via the ExoMol database. The only $\mathrm{AlCl}$ collisional rates we found were for $\mathrm{AlCl}-\mathrm{He}$ collisions by Pamboundom et al. (2016). However, the rates calculated in that study only go up to the $J=17, v=0$ rotational level of $\mathrm{AlCl}$, whereas our ALMA observations cover the $J=18 \rightarrow 17, v=0$ transition. Hence, using the rates of Pamboundom et al. (2016) would not give us the most accurate model results, even if they were scaled to account for the different mass of the $\mathrm{AlCl}-\mathrm{H}_{2}$ system.

We searched for available rates for a molecule with similar characteristics to $\mathrm{AlCl}$. Via the BASECOL ${ }^{12}$ database (Dubernet et al. 2013), we found rates calculated by Kłos \& Lique (2008) for collisions between $\mathrm{SiS}-\mathrm{H}_{2}$, considering both ortho- and para$\mathrm{H}_{2}$ and going up to the $J=40$ rotational level, in the ground vibrational state. SiS has a dipole moment of $1.74 \mathrm{D}$ (Hoeft et al. 1969; Murty \& Curl 1969), very close to the dipole moment of AlCl: 1.63 D (Yousefi \& Bernath 2018). SiS and AlCl also have very similar molecular masses and comparable level energies. These similar properties suggest that the collisional rates of SiS with $\mathrm{H}_{2}$ are an adequate stand-in for the collisional rates of $\mathrm{AlCl}$ with $\mathrm{H}_{2}$. For our model, we weighted the rates assuming an $\mathrm{H}_{2}$ ortho-to-para ratio of 3 , as is typical for warm environments.

\section{Appendix B.2: AlF}

For AlF we include molecular data for levels with $J \leq 30$ and $v \leq 6$. As for $\mathrm{AlCl}$, the maximum vibrationally excited level was chosen for its term energy, which is also $2.2 \mu \mathrm{m}$, close to the peak flux of W Aql. We neglect the hyperfine structure of AlF because the separation of the hyperfine components is smaller than the spectral resolution of our observations (based on parameters calculated by Wyse et al. 1970). The radiative information used in our AlF models comes from Yousefi \& Bernath (2018), accessed via the ExoMol database.

For the collisional rates, a new calculation was performed to obtain rates for sufficiently high temperatures as seen in our circumstellar model. This calculation is an extension of the earlier work by Gotoum et al. (2012), in which rates were given for temperatures up to $70 \mathrm{~K}$. Using the potential energy surface (PES) for AlF- $\mathrm{H}_{2}$ given by Gotoum et al. (2012), we broadened the calculations of the integral cross-sections for kinetic energy up to $10000 \mathrm{~cm}^{-1}$. Indeed the earlier work was limited for total energy $E_{c} \leq 350 \mathrm{~cm}^{-1}$ where cross-sections were calculated in the quantum mechanical close coupling formalism (CC, Arthurs \& Dalgarno 1960). We broadened the calculation up to $500 \mathrm{~cm}^{-1}$ using the CC method with $J_{\max }=20$, then up to $10000 \mathrm{~cm}^{-1}$ using the coupled-state (CS) approach (McGuire \& Kouri 1974) with $J_{\max }=50$. The following energy steps were considered, where all values are in $\mathrm{cm}^{-1}$ : 1 for $350 \leq E \leq 500,5$ for $500 \leq E \leq 1000,20$ for $1000 \leq E \leq 2000$ and finally with uniform steps of 50 up to 10000 . All these computations are done using the MOLSCAT package (Hutson \& Green 2012).

In Fig. B.1, we present the rotational quenching crosssections of $\mathrm{AlF}$ in collision with $\mathrm{H}_{2}$ for the $J=1$ to 12 rotational levels. This figure shows that for low kinetic energies crosssections present shape and Feshbach resonances and a large overlap between them. The quenching cross-sections for $J=1$ and $J=2$ are separated from the others from $E_{c}=20 \mathrm{~cm}^{-1}$, while the others explode at around 500 to $1000 \mathrm{~cm}^{-1}$.

\footnotetext{
12 https://basecol.vamdc.eu
} 
Table A.1. Measured rms values for undetected lines of $\mathrm{NaCl}, \mathrm{KCl}, \mathrm{AlO}$, and $\mathrm{AlOH}$ towards $\mathrm{W}$ Aql.

\begin{tabular}{cccccc}
\hline \hline Molecule & $\begin{array}{c}\text { Transition } \\
(v=0)\end{array}$ & $\begin{array}{c}\text { Frequency } \\
{[\mathrm{GHz}]}\end{array}$ & $\begin{array}{c}\text { Extended }\left(R=0 .^{\prime} 1\right) \\
{[\mathrm{mJy}]}\end{array}$ & $\begin{array}{c}\text { Mid }\left(R=0 .^{\prime} 6\right) \\
{[\mathrm{mJy}]}\end{array}$ & $\begin{array}{c}\text { Vel res } \\
{\left[\mathrm{km} \mathrm{s}^{-1}\right]}\end{array}$ \\
\hline $\mathrm{NaCl}$ & $(17 \rightarrow 16)$ & 221.260 & 4.1 & 4.9 & 1.3 \\
$\mathrm{NaCl}$ & $(19 \rightarrow 18)$ & $247.240^{a}$ & 4.5 & 6.0 & 1.2 \\
$\mathrm{NaCl}$ & $(20 \rightarrow 19)$ & 260.223 & $\begin{array}{c}\text { Overlap with } \mathrm{H}^{13} \mathrm{CN} \\
(3-2) v_{2}=1\end{array}$ & $\ldots$ \\
$\mathrm{KCl}$ & $(28 \rightarrow 27)$ & 215.008 & 4.0 & 4.4 & 1.4 \\
$\mathrm{KCl}$ & $(30 \rightarrow 29)$ & 230.321 & 3.8 & 4.3 & 1.3 \\
$\mathrm{KCl}$ & $(32 \rightarrow 31)$ & $245.624^{b}$ & 5.6 & 5.6 & 1.2 \\
$\mathrm{KCl}$ & $(33 \rightarrow 32)$ & $253.271^{b}$ & 3.8 & 7.1 & 1.2 \\
$\mathrm{KCl}$ & $(35 \rightarrow 34)$ & 268.559 & 6.5 & 12.3 & 1.1 \\
$\mathrm{AlO}$ & $(6 \rightarrow 5)$ & 229.670 & 3.3 & 4.9 & 1.3 \\
$\mathrm{AlO}$ & $(7 \rightarrow 6)$ & 267.937 & 6.4 & 11.7 & 1.1 \\
$\mathrm{AlOH}$ & $(7 \rightarrow 6)$ & 220.330 & 3.4 & 3.7 & 1.3 \\
$\mathrm{AlOH}$ & $(8 \rightarrow 7)$ & 251.794 & 4.1 & 8.3 & 1.2 \\
\hline
\end{tabular}

Notes. Wavelengths and energies taken from CDMS for NaCl: (Clouser \& Gordy 1964; Uehara et al. 1989; Caris et al. 2002; Timp et al. 2012; Cabezas et al. 2016); KCl: (Clouser \& Gordy 1964; Caris et al. 2004; Barton et al. 2014); AlO: Törring \& Herrmann (1989); Yamada et al. (1990); and from the JPL Molecular Spectroscopy Database for AlOH: Apponi et al. (1993). ${ }^{(a)}$ Line falls on the edge of a band, and rms is measured nearby, rather than at the line frequency, so as to exclude artefacts on the band edge. ${ }^{(b)}$ Due to nearby lines, rms is measured only to one side of the listed line, to avoid contamination.

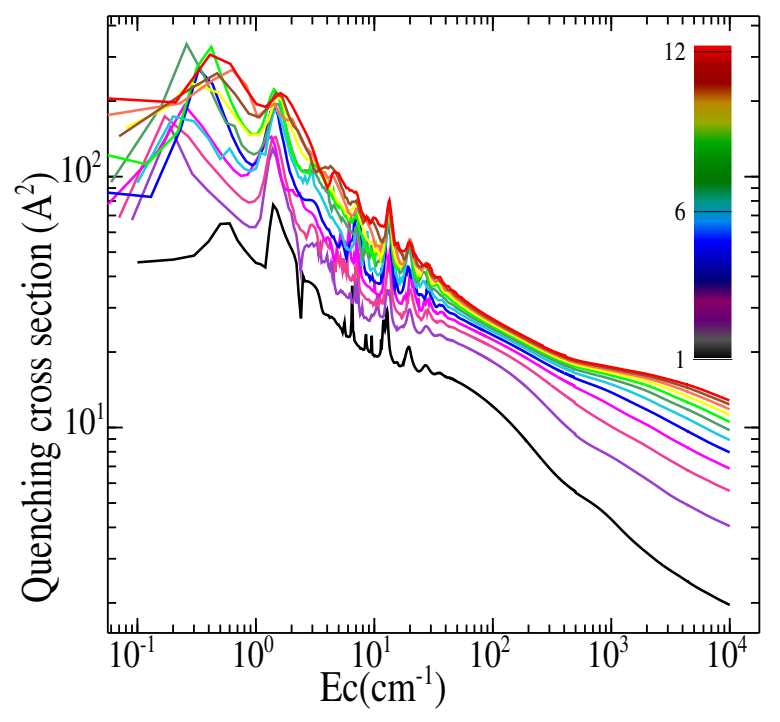

Fig. B.1. Rotational quenching cross-sections of AlF induced by collision with $\mathrm{H}_{2}$ as a function of the kinetic energy, presented with colour diagram, with $J=1$ in black to $J=12$ in red.

Generally, as the rotational level increases, the energy gap of the rotational transitions increases, and the efficiency of the rotational quenching decreases. That is why the quenching crosssections vary only slightly as a function of rotational levels for $J>2$.

These cross-sections were averaged over the Boltzmann distribution of velocities to determine the downward rate coefficients of $\mathrm{AlF}$ in collision with $\mathrm{H}_{2}$ for kinetic temperature up to $2000 \mathrm{~K}$. We present in Fig. B.2 these rates as a function of temperature for $\Delta J=-1$, with $J$ from 1 to 12 . The rate curves exhibit the same trends and increase with increasing $J$, and the gap between the plots narrow considerably. In addition, the collision rate coefficients reflect the same behaviour as the general trends observed previously for its valence isomers: $\mathrm{AlOH}-\mathrm{H}_{2}$ (Naouai et al. 2019) and HCP-H $\mathrm{H}_{2}$ (Hammami et al. 2008). We should note

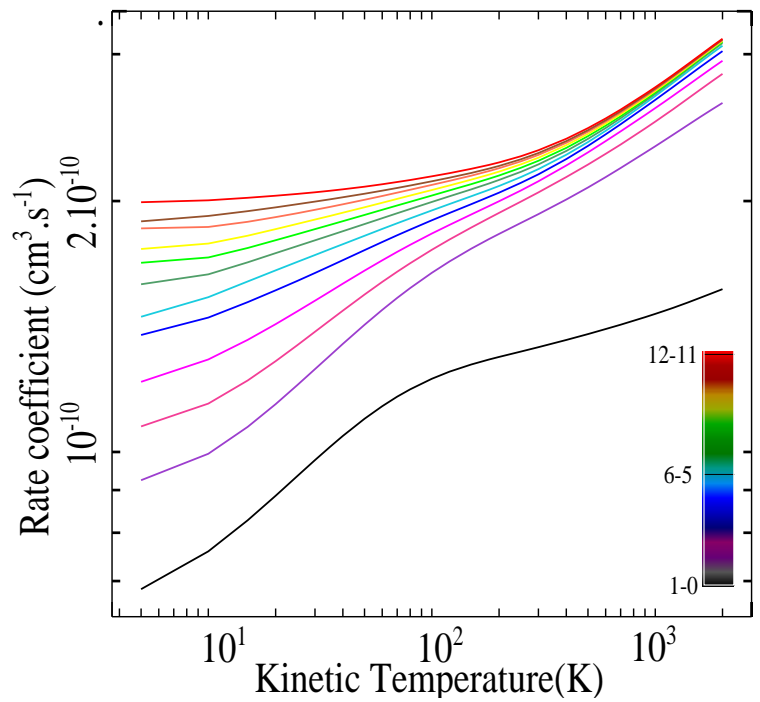

Fig. B.2. Log-log scale variation in downward rate coefficients for transitions with $\Delta J=-1$, as a function of the kinetic temperature.

here that the reason this latter behaviour is also observed for the quenching cross-sections for high energy values is probably because of the small rotational constant of AlF $(\sim 0.5)$. In this case the kinetic energy is very large compared to the rotational energies and hence the statistical approach can be valid.

\section{Appendix B.3: $\mathrm{HCl}$}

For $\mathrm{HCl}$ we include molecular data for levels with $J \leq 16$ and $v \leq 2$, neglecting hyperfine structure since our PACS observations are spectrally unresolved. The spectroscopic information used in our models of $\mathrm{HCl}$ comes from Gordon et al. (2017). We used HCl- $\mathrm{H}_{2}$ collisional rates from Lanza et al. (2014) accessed from BASECOL (Dubernet et al. 2013). Lanza et al. (2014) calculate rates for both ortho- and para- $\mathrm{H}_{2}$ and we again weighted these assuming an $\mathrm{H}_{2}$ ortho-to-para ratio of 3 . 


\section{Appendix B.4: HF}

For HF we use a molecular data file obtained from the LAMDA database $^{13}$ (Schöier et al. 2005; van der Tak et al. 2020), which includes levels up to $J \leq 8$ for $v=0$ and up to $J \leq 6$ for $v=1$. The data for the ground vibrational state was taken from Nolt et al. (1987) via the JPL Molecular Spectroscopy Database ${ }^{14}$ (Pickett et al. 1998). Rovibrational transition frequencies came from Goddon et al. (1991) and Einstein A values were computed from Pine et al. (1985). We used the collisional rates for $\mathrm{HF}-\mathrm{H}_{2}$ calculated by Guillon \& Stoecklin (2012), again assuming an $\mathrm{H}_{2}$ ortho-to-para ratio of 3.

\section{Appendix C: $\chi$ Cyg HCl and $\mathrm{HF}$}

As noted in Sect. 6.1.1, we found evidence of $\mathrm{HCl}$ and $\mathrm{HF}$ emission in the PACS spectrum of $\chi$ Cyg. To be able to directly compare the abundances of $\mathrm{HCl}$ and $\mathrm{HF}$ between $\chi \mathrm{Cyg}$ and $\mathrm{W} \mathrm{Aql}$, we also ran radiative transfer models for $\chi \mathrm{Cyg}$, using the same methods we used for $\mathrm{W}$ Aql in Sections 5.3.1 and 5.3.2. For the $\mathrm{HCl}$ and $\mathrm{HF}$ abundance distributions, we re-ran the chemical model described in Sect. 4.3, adjusted for the mass-loss rate and expansion velocity of $\chi$ Cyg $\left(\dot{M}=7 \times 10^{-7} \mathrm{M}_{\odot} \mathrm{yr}^{-1}\right.$ and $v_{\infty}=8.5 \mathrm{~km} \mathrm{~s}^{-1}$, Schöier et al. 2011). The stellar and circumstellar parameters for $\chi$ Cyg were taken from Schöier et al. (2011). As for W Aql, we used the non-detected lines to constrain the upper limits of the models.

For the $\mathrm{HCl}$ model, we found that the results were more strongly dependent on the choice of inner radius for $\chi$ Cyg than they were for $\mathrm{W}$ Aql. The inner radius used by Schöier et al. (2011) $R_{\text {in }}=2 \times 10^{14} \mathrm{~cm}$ resulted in models that significantly under-predited the higher- $J \mathrm{HCl}$ lines. When we treated the inner radius as a free parameter, we found the best fitting model to have $R_{\text {in }}=1.2 \times 10^{14} \mathrm{~cm}$ and abundances of $4.6 \times 10^{-8}$ for $\mathrm{H}^{35} \mathrm{Cl}$ and $1.9 \times 10^{-8}$ for $\mathrm{H}^{37} \mathrm{Cl}$. The ${ }^{35} \mathrm{Cl} /{ }^{37} \mathrm{Cl}$ ratio was fixed based on our $\mathrm{W}$ Aql AlCl results, since the PACS data are not of sufficiently high quality to independently determine the isotopic ratio. The PACS spectra of $\mathrm{HCl}$ are plotted with the model $\mathrm{HCl}$ lines in Fig. C.1 and the radial abundance profile is shown in Fig. C.2. The fact that changing the $R_{\text {in }}$ from $2 \times 10^{14} \mathrm{~cm}$ to $1.2 \times 10^{14} \mathrm{~cm}$ had little effect on the intensity of the $(3 \rightarrow 2)$ model line but an increasingly significant effect on the higher$J$ lines suggests that these lines are probably emitted from the $1.2-2 \times 10^{14} \mathrm{~cm}$ region. However, higher quality observations, preferably spectrally and spatially resolved, are needed to confirm this.

For HF, we found that the $(3 \rightarrow 2)$ and $(4 \rightarrow 3)$ lines were more clearly seen towards $\chi$ Cyg than $\mathrm{W}$ Aql, possibly due to the closer proximity of that star ( $150 \mathrm{pc}$ for $\chi$ Cyg compared with $395 \mathrm{pc}$ for W Aql). As for W Aql, the $(2 \rightarrow 1)$ line towards $\chi$ Cyg is blended with $\mathrm{H}_{2} \mathrm{O}$. However, although Schöier et al. (2011) modelled $\mathrm{H}_{2} \mathrm{O}$ towards $\chi$ Cyg (to find an abundance about $80 \%$ of the $\mathrm{W}$ Aql $\mathrm{H}_{2} \mathrm{O}$ abundance), their model was based only on HIFI observations and did not include any PACS data. Hence we are unable to estimate the contribution of $\mathrm{H}_{2} \mathrm{O}$ to the $\mathrm{H}_{2} \mathrm{O}+\mathrm{HF}$ line blend, as was done for $\mathrm{W}$ Aql. Instead, we focus our HF modelling on the other two HF lines, which are not known to be blended. Similar to $\mathrm{HCl}$, we found that the line ratios of these two HF lines were very sensitive to the choice of inner radius. Leaving $R_{\text {in }}$ as a free parameter again, we found the best fitting model had $R_{\text {in }}=6 \times 10^{13} \mathrm{~cm} \approx 2 R_{\star}$ and an inner HF abundance

\footnotetext{
13 https://home.strw. leidenuniv.nl/ moldata/

14 https://spec.jpl.nasa.gov/home.html
}
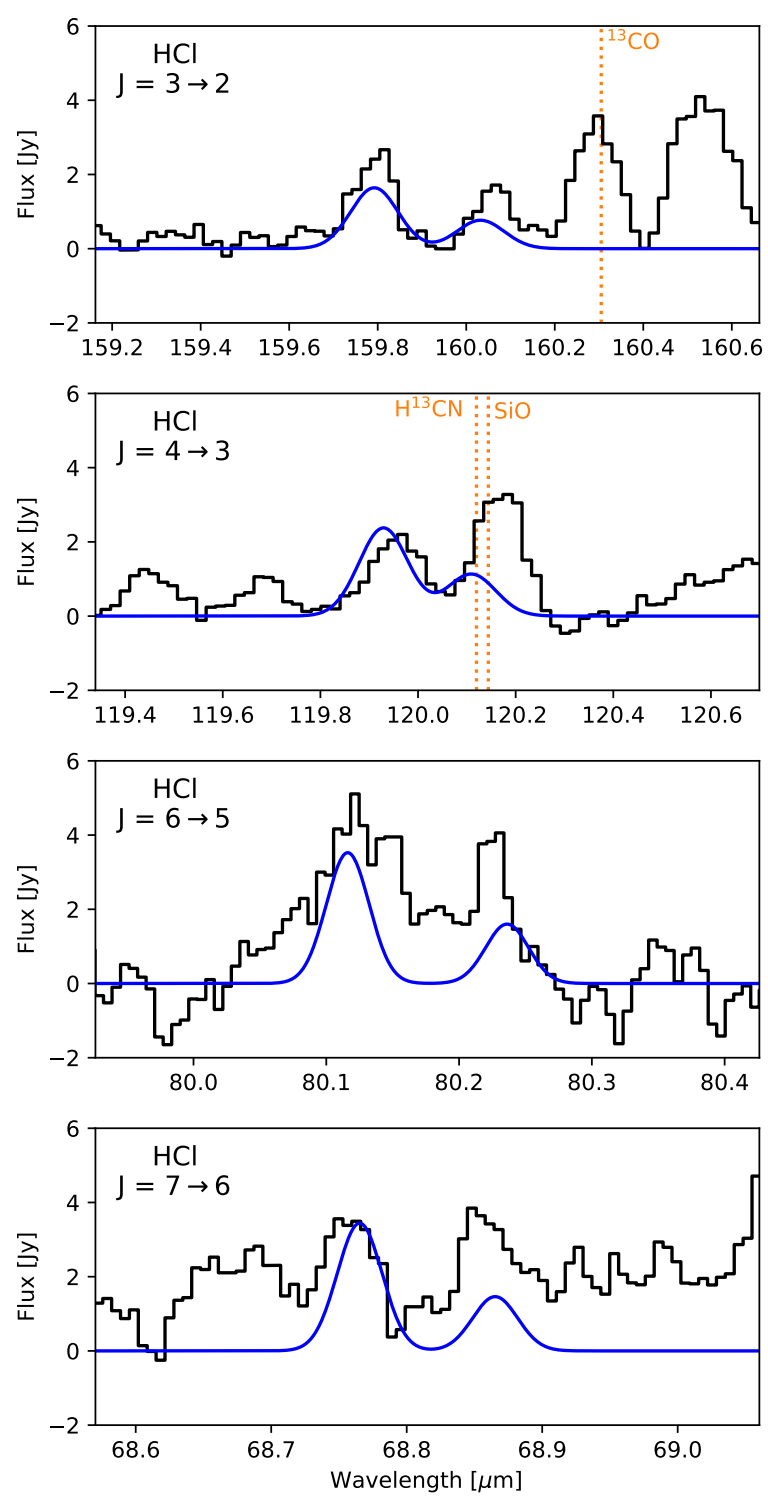

Fig. C.1. PACS spectra (black histograms) and model results (blue curves) for $\mathrm{HCl}$ towards $\chi$ Cyg. For each pair of lines, $\mathrm{H}^{35} \mathrm{Cl}$ is shown on the left since it has the shorter wavelength and $\mathrm{H}^{37} \mathrm{Cl}$ is on the right, with the longer wavelength. Some known nearby and blended lines are indicated in orange (but not all nearby lines have been identified).

of $1.2 \times 10^{-8}$ relative to $\mathrm{H}_{2}$. These best fitting model lines are plotted with the PACS spectra of HF in Fig. C.3 and the radial abundance profile is shown in Fig. C.2.

\section{Appendix D: Supplementary information concerning the chemistry of AIF and AICl}

The rate coefficients for the reactions of $\mathrm{Al}, \mathrm{AlO}$ and $\mathrm{AlOH}$ with $\mathrm{HF}$ and $\mathrm{HCl}$ to produce $\mathrm{AlF}$ and $\mathrm{AlCl}$, respectively, were estimated by combining electronic structure calculations with RiceRamsperger-Kassel-Markus (RRKM) statistical rate theory. Accurate energies, geometries and vibrational frequencies were determined at the G4 level of theory (Curtiss et al. 2007) within the Gaussian 16 suite of programs (Frisch et al. 2016). The Cartesian coordinates, rotational constants, vibrational frequencies and heats of formation of the relevant molecules are listed in Table D.2 for reactions producing AlF (R1 - R3), and Table D.3 


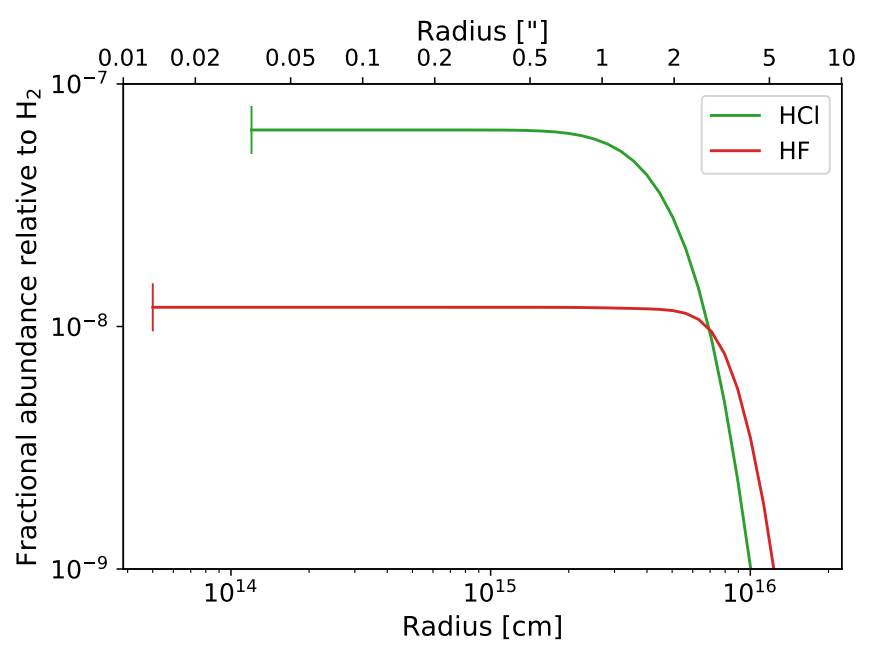

Fig. C.2. Radial abundance profiles for $\mathrm{HCl}$ (green) and $\mathrm{HF}$ (red) as derived for $\chi$ Cyg. The short vertical lines indicate the inner radius of the corresponding model.

Table D.1. Capture rate coefficients calculated using long range transition state theory (Georgievskii \& Klippenstein 2005).

\begin{tabular}{lcc}
\hline \hline & Reaction & $\begin{array}{c}\text { Rate coefficient } \\
{\left[\mathrm{cm}^{3} \text { molecule }^{-1} \mathrm{~s}^{-1}\right]}\end{array}$ \\
\hline $\mathrm{R} 1$ & $\mathrm{Al}+\mathrm{HF} \rightarrow \mathrm{AlF}+\mathrm{H}$ & $7.7 \times 10^{-10} \exp (-86 / T)$ \\
$\mathrm{R} 2$ & $\mathrm{AlOH}+\mathrm{HF} \rightarrow \mathrm{AlF}+\mathrm{H}_{2} \mathrm{O}$ & $7.8 \times 10^{-10} \exp (-86 / T)$ \\
$\mathrm{R} 3$ & $\mathrm{AlO}+\mathrm{HF} \rightarrow \mathrm{AlF}+\mathrm{OH}$ & $1.0 \times 10^{-9}(T / 298)^{-0.17}$ \\
$\mathrm{R} 4$ & $\mathrm{Al}+\mathrm{HCl} \rightarrow \mathrm{AlCl}+\mathrm{H}$ & $1.0 \times 10^{-9} \exp (-86 / T)$ \\
$\mathrm{R} 5$ & $\mathrm{AlOH}+\mathrm{HCl} \rightarrow \mathrm{AlCl}+\mathrm{H}_{2} \mathrm{O}$ & $1.0 \times 10^{-9} \exp (-86 / T)$ \\
$\mathrm{R} 6$ & $\mathrm{AlO}+\mathrm{HCl} \rightarrow \mathrm{AlCl}+\mathrm{OH}$ & $1.0 \times 10^{-9} \exp (-86 / T)$ \\
\hline
\end{tabular}

for the $\mathrm{AlCl}$-forming reactions ( $\mathrm{R} 4-\mathrm{R} 6)$. The potential energy surfaces for the six reactions are illustrated in Figs. D.1 and D.2, which also show the geometries of the stationary points. We note that the relative energies include zero-point energy corrections.

The Master Equation Solver for Multi-Energy well Reactions (MESMER) program (Glowacki et al. 2012) was then used to estimate rate coefficients. Apart from R4, these reactions proceed via the formation of an energised adduct. This adduct can dissociate back to the reactants, dissociate to bimolecular products sometimes involving a barrier (R1, R2, and R5, but not R3 or R6), or be stabilised by collision with a third body (which in the case of the outflow is $\mathrm{H}_{2}$ ). The internal energies of the stationary points on the surface (i.e. reactants, intermediates, transition states and products) were divided into a contiguous set of grains (width $=150 \mathrm{~cm}^{-1}$ ) containing a bundle of rovibrational states. The density of states of each stationary point was calculated using the vibrational frequencies and rotational constants listed in Tables D.2 and D.3. The vibrations were treated as harmonic oscillators, and a classical densities of states treatment was used for the rotational modes.

Each adduct grain was then assigned a set of microcanonical rate coefficients for dissociation to the reactants and products. These were determined using inverse Laplace transformation to link them directly to the relevant capture rates. These capture rates were calculated using long-range transition state theory (Georgievskii \& Klippenstein 2005), and are listed in Table D.1.

The probability of collisional transfer between grains was estimated using the exponential down model (Gilbert \& Smith
1990): the average energy for downward transitions, $\langle\Delta E\rangle_{\text {down }}$, was set to $200 \mathrm{~cm}^{-1}$ with no temperature dependence, and the probabilities for upward transitions are determined by detailed balance. The collision rate of $\mathrm{H}_{2}$ with the adduct as a function of temperature was calculated using Lennard-Jones parameters $(\sigma=3.0 \AA$ and $\varepsilon / k=200 \mathrm{~K})$ to characterise the intermolecular potential. The ME, which describes the evolution with time of the adduct grain populations, was then expressed in matrix form and solved to yield the rate coefficients for recombination and bimolecular reaction at a specified pressure and temperature. In fact, at the low pressures in the outflow $\left(\left[\mathrm{H}_{2}\right]<10^{8} \mathrm{~cm}^{-3}\right.$ beyond a radius of $2 \times 10^{14} \mathrm{~cm}$ ), it is only the bimolecular channels that matter. The rate coefficients for the reverse reactions were calculated by detailed balance, using the molecular parameters in Tables D. 2 and D. 3 to calculate the relevant equilibrium constants. In the case of reaction $\mathrm{R} 6$ between $\mathrm{AlO}$ and $\mathrm{HCl}$, the $\mathrm{AlOH}+\mathrm{Cl}$ channel is $44 \mathrm{~kJ} \mathrm{~mol}^{-1}$ more exothermic than $\mathrm{AlCl}+$ $\mathrm{OH}$, and so $\mathrm{AlOH}$ is the major product $(91 \%$ at $900 \mathrm{~K}, 79 \%$ at $1900 \mathrm{~K})$.

The absorption cross-sections for $\mathrm{AlF}$ and $\mathrm{AlCl}$ were calculated by first optimising their geometries at the B3LYP/6$311+\mathrm{g}(2 \mathrm{~d}, \mathrm{p})$ level of theory (Frisch et al. 2016), before determining the vertical excitation energies and transition dipole moments for transitions from their ground electronic states to the first 50 electronically excited states, using the time-dependent density function theory (TD-DFT) method (Bauernschmitt \& Ahlrichs 1996). The resulting cross-sections are illustrated in Fig. D.3. Because the AlF bond strength is so large $\left(681 \mathrm{~kJ} \mathrm{~mol}^{-1}\right.$ at the G4 level of theory, Curtiss et al. 2007) compared with that of $\mathrm{AlCl}\left(507 \mathrm{~kJ} \mathrm{~mol}^{-1}\right)$, the wavelength threshold for photolysis of AlF is $175 \mathrm{~nm}$ compared with $235 \mathrm{~nm}$ for AlCl. Interstellar radiation is attenuated by dust within the outflow, where the extinction is assumed to be equal to that of the ISM, namely $1.87 \times 10^{21}$ atoms $\mathrm{cm}^{-2} \mathrm{mag}^{-1}$ (Cardelli et al. 1989).

The outflow model was initialised with the parent species listed in Table D.4. Additional reactions, beyond those published in McElroy et al. (2013) as part of RATE12 are listed in Table D.5. The tabulated constants give the rate coefficient via a temperature-dependent Arrhenius-type formula:

$k=\alpha\left(\frac{T}{300}\right)^{\beta} \exp \left(\frac{-\gamma}{T}\right) \mathrm{cm}^{3} \mathrm{~s}^{-1}$,

except for reactions with (interstellar) photons $(\mathrm{h} v)$, which are parameterised as

$k=\alpha \exp \left(-\gamma A_{V}\right) \mathrm{s}^{-1}$,

where $A_{V}$ is the dust extinction at visible wavelengths.

The rate coefficients for R1 to R6 were adjusted by varying the heights of the transition states within the expected uncertainty of the G4 level of theory $\left(6 \mathrm{~kJ} \mathrm{~mol}^{-1}\right)$ to optimise agreement with the observations in Fig. 12. These rate coefficients are listed in Table D.1. 

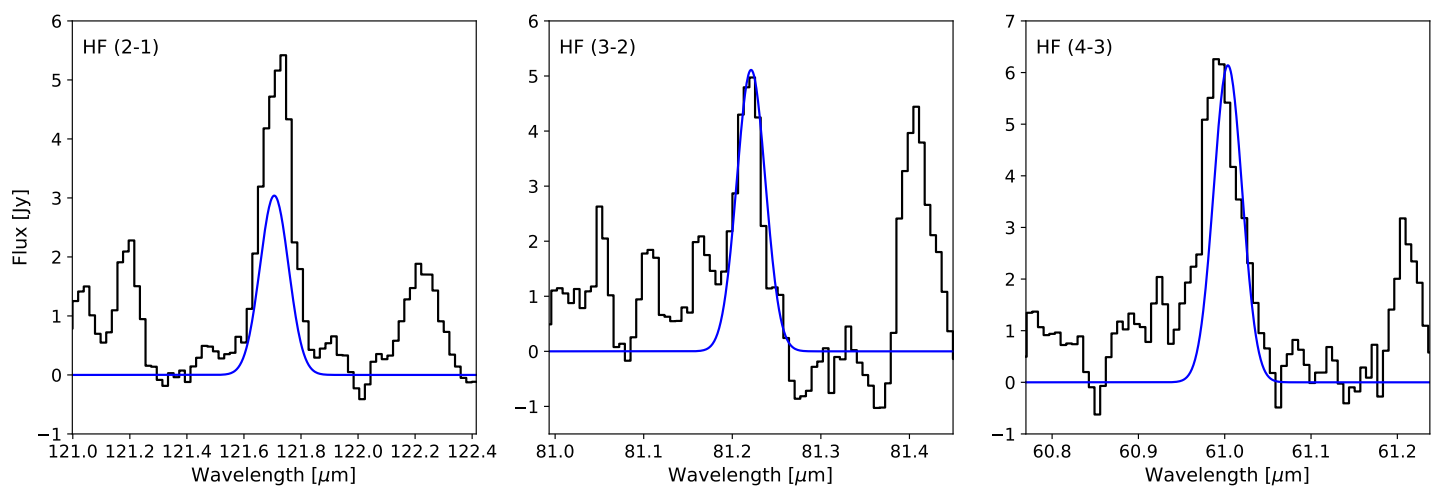

Fig. C.3. PACS spectra (black histograms) and model results (blue curves) for HF towards $\chi$ Cyg. The $\mathrm{HF}(2 \rightarrow 1)$ line is blended with the o- $\mathrm{H}_{2} \mathrm{O}$ line at $121.721 \mu \mathrm{m}$, which is not shown here. See text for details.

Table D.2. Molecular properties and heats of formation (at $0 \mathrm{~K}$ ) of the stationary points on the $\mathrm{Al}, \mathrm{AlOH}$ and $\mathrm{AlO}+\mathrm{HF}$ potential energy surfaces.

\begin{tabular}{|c|c|c|c|c|}
\hline $\begin{array}{c}\text { Molecule } \\
\text { (electronic state) }\end{array}$ & $\begin{array}{c}\text { Geometry }^{a} \\
{[\text { Cartesian co-ords in } \AA]}\end{array}$ & $\begin{array}{c}\text { Rotational constants }^{a} \\
{[\mathrm{GHz}]}\end{array}$ & $\begin{array}{l}\text { Vibrational freq. }^{a} \\
{\left[\mathrm{~cm}^{-1}\right]}\end{array}$ & $\begin{array}{c}\delta_{f} \mathrm{H}^{o}(0 \mathrm{~K})^{b} \\
{\left[\mathrm{~kJ} \mathrm{~mol}{ }^{-1}\right]}\end{array}$ \\
\hline \multicolumn{5}{|c|}{$\mathrm{Al}+\mathrm{HF} \rightarrow \mathrm{AlF}+\mathrm{H}$} \\
\hline $\mathrm{HF}$ & $\begin{array}{l}\text { F, 0., 0., } 0.090 \\
\text { H, 0., 0., - }-0.829\end{array}$ & 624.62 & 4117 & -273.6 \\
\hline Al-HF complex & $\begin{array}{l}\mathrm{Al}, 0.151,-1.228,0 . \\
\mathrm{H},-0.944,0.847,0 . \\
\mathrm{F},-0.032,1.098,0 .\end{array}$ & 607.478 .13478 .0272 & $159,467,3541$ & 47.3 \\
\hline $\begin{array}{l}\text { TS from Al-HF complex } \\
\text { to AlF + H (TS1) }\end{array}$ & $\begin{array}{l}\text { Al, } 0.048,-0.843,0 . \\
H,-0.991,0.757,0 . \\
F, 0.042,1.143,0 .\end{array}$ & 485.8311 .33011 .072 & $894.2 i, 519,1418$ & 56.2 \\
\hline $\mathrm{AlF}$ & $\begin{array}{l}\text { F, 0., 0., }-0.983 \\
\text { Al, 0., 0., } 0.677\end{array}$ & 16.4524 & 828 & -275.0 \\
\hline $\mathrm{AlOH}$ & $\begin{array}{l}\mathrm{AlOH}+\mathrm{H} \\
\mathrm{Al}, 0.026,0 ., 0.018 \\
\mathrm{O},-0.063,0 ., 1.702 \\
\mathrm{H}, 0.364,0 ., 2.555\end{array}$ & $\begin{array}{l}\rightarrow \mathrm{AlF}+\mathrm{H}_{2} \mathrm{O} \\
2590.615 .75415 .658\end{array}$ & $215,849,3960$ & -186.6 \\
\hline AlOH-HF complex & $\begin{array}{l}\text { Al, }-0.226,-0.705,0.001 \\
\mathrm{O},-0.202,1.069,-0.003 \\
\mathrm{H},-0.888,1.736,0.002 \\
\mathrm{~F}, 1.893,0.063,0.001 \\
\mathrm{H}, 1.347,0.865,-0.000\end{array}$ & 14.3018 .22975 .2238 & $\begin{array}{l}131,139,338, \\
559,705,793 \\
1080,3151,3919\end{array}$ & -471.3 \\
\hline $\begin{array}{l}\text { TS from AlOH-HF } \\
\text { complex to } \mathrm{AlF}+\mathrm{H}_{2} \mathrm{O}\end{array}$ & $\begin{array}{l}\mathrm{Al}, 0.525,-0.801,0.021 \\
\mathrm{O}, 0.640,1.020,-0.069 \\
\mathrm{H},-0.714,0.845,0.014 \\
\mathrm{~F},-1.368,0.037,-0.001 \\
\mathrm{H}, 1.217,1.647,0.371\end{array}$ & 13.4439 .76645 .6824 & $\begin{array}{l}-263 i, 267,399, \\
586,674,1030 \\
1237,2187,3901\end{array}$ & -465.8 \\
\hline $\mathrm{H}_{2} \mathrm{O}$ & $\begin{array}{l}\mathrm{O}, 0.001,0 ., 0.001 \\
\mathrm{H} 0,0.0123,0 ., 0.963 \\
\mathrm{H}, 0.933,0 .,-0.237\end{array}$ & 798.21438 .23282 .91 & $1672,3802,3906$ & -247.2 \\
\hline $\mathrm{AlO}$ & $\begin{array}{l}\mathrm{Al}, 0 ., 0 ., 0.002 \\
\mathrm{O}, 0 ., 0 ., 1.628\end{array}$ & $\begin{array}{l}\rightarrow \mathrm{AlF}+\mathrm{OH} \\
19.0158\end{array}$ & 967 & 70.3 \\
\hline $\mathrm{FAlOH}$ & $\begin{array}{l}\mathrm{Al}, 0.704,0.261,0 . \\
\mathrm{O},-0.923,-0.219,0 . \\
\mathrm{H},-1.179,-1.144,0 . \\
\mathrm{F}, 1.949,-0.829,0 .\end{array}$ & 43.5026 .51575 .6669 & $\begin{array}{l}215,308,622 \\
786,923,3873\end{array}$ & -556.4 \\
\hline $\mathrm{OH}$ & $\begin{array}{l}\mathrm{O}, 0 ., 0 ., 0.002 \\
\mathrm{H}, 0 ., 0 ., 0.978\end{array}$ & 559.17 & 3691 & 34.8 \\
\hline
\end{tabular}

Notes. TS denotes a transition state. $\left({ }^{a}\right)$ Calculated at the G4 level of theory (Curtiss et al. 2007; Frisch et al. 2016). $\left({ }^{b}\right)$ Calculated at the G4 level of theory (Curtiss et al. 2007; Frisch et al. 2016) with JANAF reference values for $\Delta_{f} H^{o}(\mathrm{Al})=327.3 \mathrm{~kJ} \mathrm{~mol}^{-1}, \Delta_{f} H^{o}(\mathrm{O})=246.8 \mathrm{~kJ}$ mol ${ }^{-1}$, $\Delta_{f} H^{o}(\mathrm{~F})=77.3 \mathrm{~kJ} \mathrm{~mol}^{-1}$, and $\Delta_{f} H^{o}(\mathrm{H})=216.0 \mathrm{~kJ} \mathrm{~mol}^{-1}$ (Chase et al. 1985). 
T. Danilovich et al.: ATOMIUM: Halide molecules around the S-type AGB star W Aquilae

Table D.3. Molecular properties and heats of formation (at $0 \mathrm{~K}$ ) of the stationary points on the $\mathrm{Al}, \mathrm{AlOH}$ and $\mathrm{AlO}+\mathrm{HCl}$ potential energy surfaces.

\begin{tabular}{|c|c|c|c|c|}
\hline $\begin{array}{c}\text { Molecule } \\
\text { (electronic state) }\end{array}$ & $\begin{array}{c}\text { Geometry }^{a} \\
\text { [Cartesian co-ords in } \AA \text { ] }\end{array}$ & $\begin{array}{c}\text { Rotational constants }^{a} \\
{[\mathrm{GHz}]}\end{array}$ & $\begin{array}{l}\text { Vibrational freq. }^{a} \\
{\left[\mathrm{~cm}^{-1}\right]}\end{array}$ & $\begin{array}{l}\delta_{f} \mathrm{H}^{o}(0 \mathrm{~K})^{b} \\
{\left[\mathrm{~kJ} \mathrm{~mol}^{-1}\right]} \\
\end{array}$ \\
\hline $\mathrm{HCl}$ & $\begin{array}{l}\mathrm{H}, 0 ., 0 .,-1.212 \\
\mathrm{Cl}, 0 ., 0 ., 0.072\end{array}$ & 313.07 & 2958 & -90.3 \\
\hline $\begin{array}{l}\mathrm{TS} \text { from } \mathrm{Al}+\mathrm{HCl} \text { to } \\
\mathrm{AlCl}+\mathrm{H}(\mathrm{TS} 1)\end{array}$ & $\begin{array}{l}\mathrm{Al}, 0.036,1.415,0 . \\
\mathrm{H},-1.083,-1.946,0 . \\
\mathrm{Cl}, 0.038,-0.963,0 .\end{array}$ & 424.115 .60195 .5289 & $-801 i, 353,548$ & 248.6 \\
\hline $\mathrm{AlCl}$ & $\begin{array}{l}\mathrm{Al}, 0 ., 0 .,-1.224 \\
\mathrm{Cl}, 0 ., 0 ., 0.935\end{array}$ & 7.1141 & 463 & -59.4 \\
\hline \multicolumn{5}{|c|}{$\mathrm{AlOH}+\mathrm{HCl} \rightarrow \mathrm{AlCl}+\mathrm{H}_{2} \mathrm{O}$} \\
\hline $\begin{array}{l}\text { AlOH: see Table D. } 2 \\
\text { AlOH-HCl complex }\end{array}$ & $\begin{array}{l}\mathrm{Al},-1.825,-0.734,-0.034 \\
\mathrm{O},-0.755,0.619,-0.009 \\
\mathrm{H},-0.901,1.567,0.0228 \\
\mathrm{Cl}, 2.382,0.529,0.018 \\
\mathrm{H}, 1.072,0.540,0.003\end{array}$ & 34.3501 .62771 .5541 & $\begin{array}{l}53,145,157,437 \\
466,714,772 \\
2601,3880\end{array}$ & -290.7 \\
\hline $\begin{array}{l}\text { TS from } \mathrm{AlOH}-\mathrm{HCl} \\
\text { complex to } \mathrm{AlCl}+\mathrm{H}_{2} \mathrm{O}\end{array}$ & $\begin{array}{l}\mathrm{Al}, 1.315,-0.704,0.019 \\
\mathrm{O}, 0.929,1.071,-0.088 \\
\mathrm{H},-0.363,0.838,-0.014 \\
\mathrm{Cl},-1.494,-0.100,0.011 \\
\mathrm{H}, 1.325,1.837,0.337\end{array}$ & 13.8993 .59062 .8599 & $\begin{array}{l}-560 i, 188,232,6 \\
517,623,785,931, \\
1392,385\end{array}$ & -264.7 \\
\hline \multicolumn{5}{|l|}{$\mathrm{H}_{2} \mathrm{O}$ : see Table D.2 } \\
\hline \multicolumn{5}{|c|}{$\mathrm{AlO}+\mathrm{HCl} \rightarrow \mathrm{AlOH}+\mathrm{Cl}, \mathrm{AlCl}+\mathrm{OH}$} \\
\hline $\mathrm{ClAlOH}$ & $\begin{array}{l}\mathrm{Al}, 0.011,0.817,0 \\
\mathrm{O}, 1.697,0.979,0 . \\
\mathrm{H}, 2.296,0.228,0 . \\
\mathrm{Cl},-0.927,-1.096,0 .\end{array}$ & 32.7393 .57433 .2225 & $\begin{array}{l}179,309,469 \\
626,873,3880\end{array}$ & -351.8 \\
\hline $\begin{array}{l}\text { AlOH: see Table D. } 2 \\
\text { OH: see Table D. } 2\end{array}$ & & & & \\
\hline
\end{tabular}

Notes. TS denotes a transition state. $\left({ }^{a}\right)$ Calculated at the G4 level of theory (Curtiss et al. 2007; Frisch et al. 2016). $\left({ }^{b}\right)$ Calculated at the G4 level of theory (Curtiss et al. 2007; Frisch et al. 2016) with JANAF reference values for $\Delta_{f} H^{o}(\mathrm{Al})=327.3 \mathrm{~kJ} \mathrm{~mol}^{-1}, \Delta_{f} H^{o}(\mathrm{O})=246.8 \mathrm{~kJ}$ mol ${ }^{-1}$, $\Delta_{f} H^{o}(\mathrm{Cl})=119.6 \mathrm{~kJ} \mathrm{~mol}^{-1}$, and $\Delta_{f} H^{o}(\mathrm{H})=216.0 \mathrm{~kJ} \mathrm{~mol}^{-1}$ (Chase et al. 1985).

Table D.4. Parent species initial fractional abundances relative to $\mathrm{H}_{2}$.

\begin{tabular}{cc}
\hline Species & Abundance \\
\hline \hline $\mathrm{He}$ & 0.17 \\
$\mathrm{CO}$ & $6.2 \times 10^{-4}$ \\
$\mathrm{~N}_{2}$ & $4.0 \times 10^{-5}$ \\
$\mathrm{H}_{2} \mathrm{O}$ & $1.5 \times 10^{-5}$ \\
$\mathrm{HCN}$ & $3.3 \times 10^{-6}$ \\
$\mathrm{SiO}$ & $3.2 \times 10^{-6}$ \\
$\mathrm{NH}_{3}$ & $1.7 \times 10^{-5}$ \\
$\mathrm{SiS}$ & $1.6 \times 10^{-6}$ \\
$\mathrm{CS}$ & $1.2 \times 10^{-6}$ \\
$\mathrm{HS}$ & $1.7 \times 10^{-5}$ \\
$\mathrm{Al}$ & $5.6 \times 10^{-6}$ \\
$\mathrm{Cl}$ & $4.0 \times 10^{-7}$ \\
$\mathrm{~F}$ & $2.0 \times 10^{-7}$ \\
\hline
\end{tabular}


Table D.5. Additional reactions for halide and aluminium-bearing molecules included in our chemical modelling.

\begin{tabular}{|c|c|c|c|c|c|}
\hline Reaction & $\alpha$ & $\beta$ & $\gamma$ & Temp range $^{a}[\mathrm{~K}]$ & Source $^{b}$ \\
\hline$\overline{\mathrm{AlO}}+\mathrm{h} v \rightarrow \mathrm{Al}+\mathrm{O}$ & $3.09 \mathrm{e}-10$ & $\ldots$ & $\overline{1.7}$ & $10-3000$ & 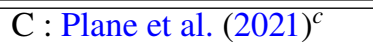 \\
\hline $\mathrm{AlCl}+\mathrm{h} v \rightarrow \mathrm{Al}+\mathrm{Cl}$ & $3.52 \mathrm{e}-10$ & $\ldots$ & 1.7 & $10-3000$ & $\mathrm{C}$ : Plane et al. $(2021)^{c}$ \\
\hline $\mathrm{AlOH}+\mathrm{h} v \rightarrow \mathrm{AlO}+\mathrm{H}$ & $4.57 e-10$ & $\ldots$ & 1.7 & $10-3000$ & C : Plane et al. (2021) \\
\hline $\mathrm{AlF}+\mathrm{h} v \rightarrow \mathrm{Al}+\mathrm{F}$ & $9.70 \mathrm{e}-11$ & $\ldots$ & 2 & $10-3000$ & $\mathrm{C}:$ This study \\
\hline $\mathrm{HF}+\mathrm{h} v \rightarrow \mathrm{H}+\mathrm{F}$ & $1.38 \mathrm{e}-10$ & $\cdots$ & 3 & $10-3000$ & M : Heays et al. (2017) \\
\hline $\mathrm{HCl}+\mathrm{h} v \rightarrow \mathrm{H}+\mathrm{Cl}$ & $1.73 \mathrm{e}-09$ & $\ldots$ & 2.88 & $10-3000$ & M : Heays et al. (2017) \\
\hline $\mathrm{Al}+\mathrm{H}_{2} \mathrm{O} \rightarrow \mathrm{AlOH}+\mathrm{H}$ & $7.66 \mathrm{e}-14$ & 3.59 & -526 & $298-1174$ & C : Mangan et al. (2021) \\
\hline $\mathrm{AlOH}+\mathrm{H} \rightarrow \mathrm{AlO}+\mathrm{H}_{2}$ & $8.89 \mathrm{e}-11$ & 0.0 & 9092 & $10-3000$ & C : Mangan et al. (2021) \\
\hline $\mathrm{AlOH}+\mathrm{H} \rightarrow \mathrm{Al}+\mathrm{H}_{2} \mathrm{O}$ & $4.31 \mathrm{e}-11$ & 0.0 & 9457 & $10-3000$ & C : Mangan et al. (2021) \\
\hline $\mathrm{Cl}+\mathrm{NH}_{3} \rightarrow \mathrm{NH}_{2}+\mathrm{HCl}$ & $1.08 \mathrm{e}-11$ & 0.0 & 1370.0 & $290-566$ & M : Gao et al. (2006) \\
\hline $\mathrm{Cl}+\mathrm{CH}_{4} \rightarrow \mathrm{CH}_{3}+\mathrm{HCl}$ & $6.60 \mathrm{e}-12$ & 0.0 & 1240.0 & $200-300$ & L : Atkinson et al. (2006) \\
\hline $\mathrm{Cl}+\mathrm{H}_{2} \mathrm{CO} \rightarrow \mathrm{HCO}+\mathrm{HCl}$ & $8.20 \mathrm{e}-11$ & 0.0 & 34.0 & $200-500$ & L : Atkinson et al. (2006) \\
\hline $\mathrm{AlO}+\mathrm{H}_{2} \rightarrow \mathrm{AlOH}+\mathrm{H}$ & $5.37 \mathrm{e}-13$ & 2.77 & 2190 & $10-3000$ & C : Mangan et al. (2021) \\
\hline $\mathrm{AlO}+\mathrm{H}_{2} \mathrm{O} \rightarrow \mathrm{AlOH}+\mathrm{OH}$ & $3.89 \mathrm{e}-10$ & 0.0 & 1295 & $10-3000$ & C : Mangan et al. (2021) \\
\hline \multirow[t]{2}{*}{$\mathrm{F}+\mathrm{H}_{2} \rightarrow \mathrm{HF}+\mathrm{H}$} & $2.54 \mathrm{e}-11$ & 1.848 & -6.182 & $20-295$ & M : Tizniti et al. (2014) \\
\hline & $1.20 \mathrm{e}-10$ & 0.0 & 470 & $295-376$ & M : Stevens et al. (1989) \\
\hline $\mathrm{Cl}+\mathrm{H}_{2} \rightarrow \mathrm{HCl}+\mathrm{H}$ & $5.27 \mathrm{e}-12$ & 1.4 & 1760 & $199-2940$ & M : Kumaran et al. (1994) \\
\hline $\mathrm{AlOH}+\mathrm{HCl} \rightarrow \mathrm{AlCl}+\mathrm{H}_{2} \mathrm{O}$ & $6 e-14$ & 1.5 & 200.0 & $100-2000$ & $\mathrm{C}:$ This study $^{d}$ \\
\hline $\mathrm{AlO}+\mathrm{HCl} \rightarrow \mathrm{AlCl}+\mathrm{OH}$ & $3.10 \mathrm{e}-10$ & 0 & 1116 & $100-2000$ & C : This study ${ }^{d}$ \\
\hline $\mathrm{AlCl}+\mathrm{H} \rightarrow \mathrm{Al}+\mathrm{HCl}$ & $1.90 \mathrm{e}-10$ & 0 & 11027 & $100-2000$ & C : This study ${ }^{d}$ \\
\hline $\mathrm{AlCl}+\mathrm{OH} \rightarrow \mathrm{AlOH}+\mathrm{Cl}$ & $3.95 \mathrm{e}-10$ & 0 & 11.3 & $10-2000$ & C : This study ${ }^{d}$ \\
\hline $\mathrm{AlCl}+\mathrm{OH} \rightarrow \mathrm{AlO}+\mathrm{HCl}$ & $3.95 \mathrm{e}-10$ & 0 & 11.3 & $10-2000$ & C : This study ${ }^{d}$ \\
\hline $\mathrm{AlCl}+\mathrm{H}_{2} \mathrm{O} \rightarrow \mathrm{AlOH}+\mathrm{HCl}$ & $7 e-14$ & 1.7 & 3200.0 & $100-2000$ & C : This study ${ }^{d}$ \\
\hline $\mathrm{Al}+\mathrm{HF} \rightarrow \mathrm{AlF}+\mathrm{H}$ & 7.7e-10 & 0.0 & 86.0 & $100-2000$ & C : This study ${ }^{d}$ \\
\hline $\mathrm{AlOH}+\mathrm{HF} \rightarrow \mathrm{AlF}+\mathrm{H}_{2} \mathrm{O}$ & $7.8 \mathrm{e}-10$ & 0.0 & 86.0 & $100-2000$ & C : This study ${ }^{d}$ \\
\hline \multirow[t]{2}{*}{$\mathrm{AlO}+\mathrm{HF} \rightarrow \mathrm{AlF}+\mathrm{OH}$} & $1.03 \mathrm{e}-09$ & 0.16 & 0 & $10-399$ & C: This study ${ }^{d}$ \\
\hline & $6.20 \mathrm{e}-10$ & 0 & -224 & $400-2000$ & C : This study ${ }^{d}$ \\
\hline $\mathrm{AlF}+\mathrm{H} \rightarrow \mathrm{Al}+\mathrm{HF}$ & $1.00 \mathrm{e}-10$ & 0 & 13756 & $100-2000$ & C : This study ${ }^{d}$ \\
\hline $\mathrm{AlF}+\mathrm{OH} \rightarrow \mathrm{AlO}+\mathrm{HF}$ & $4.50 \mathrm{e}-10$ & 0 & 4147 & $100-2000$ & C : This study ${ }^{d}$ \\
\hline $\mathrm{AlF}+\mathrm{H}_{2} \mathrm{O} \rightarrow \mathrm{AlOH}+\mathrm{HF}$ & $8.10 \mathrm{e}-10$ & 0 & 7242 & $100-2000$ & C : This study ${ }^{d}$ \\
\hline
\end{tabular}

Notes. $\left({ }^{a}\right)$ : The range over which the fit parameters are valid. $\left({ }^{b}\right)$ : C, M, and L indicate whether a fit is calculated, measured, or a literature survey value (i.e. a recommended value based on a review of available data), respectively. ${ }^{c}$ ): Calculated at the same theoretical level as the AlOH photolysis reaction in Plane et al. $(2021) .\left({ }^{d}\right)$ : The rate coefficient listed in Table 6 has been adjusted by varying the height of the transition state within the expected uncertainty of the G4 level of theory $\left(6 \mathrm{~kJ} \mathrm{~mol}^{-1}\right)$, to optimise agreement with the observations in Fig. 12. 
T. Danilovich et al.: ATOMIUM: Halide molecules around the S-type AGB star W Aquilae
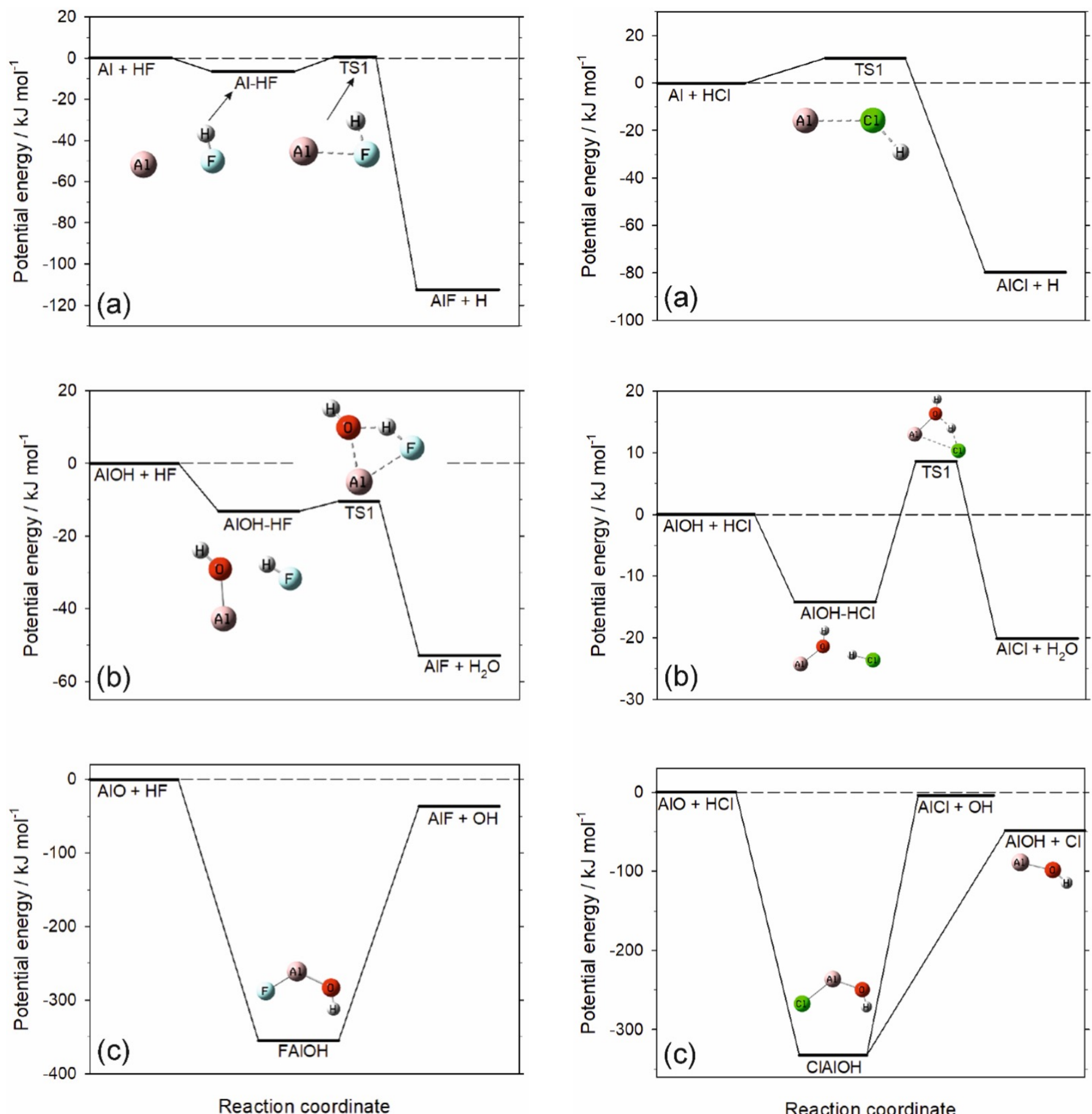

Fig. D.1. Potential energy surfaces calculated at the G4 level of theory for: (a) $\mathrm{Al}+\mathrm{HF}$ (R1); (b) $\mathrm{AlOH}+\mathrm{HF}$ (R2); (c) $\mathrm{AlO}+\mathrm{HF}$ (R3). Note that the very endothermic channels are not shown.

Fig. D.2. Potential energy surfaces calculated at the G4 level of theory for: (a) $\mathrm{Al}+\mathrm{HCl}$ (R1); (b) $\mathrm{AlOH}+\mathrm{HCl}$ (R2); (c) $\mathrm{AlO}+\mathrm{HCl}$ (R3). Note that the very endothermic channels are not shown. 
A\&A 655, A80 (2021)

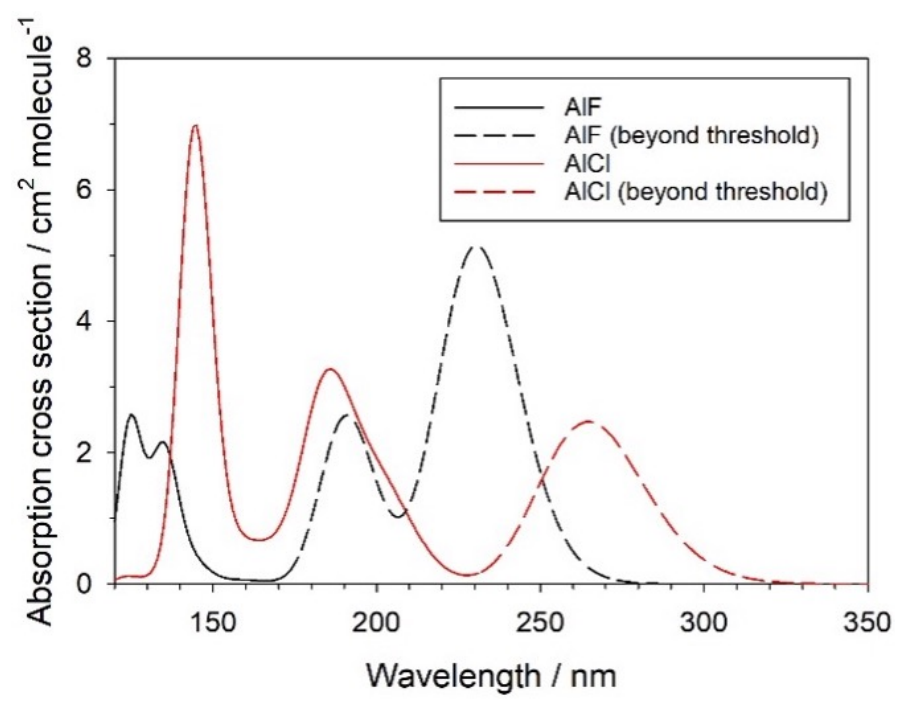

Fig. D.3. Absorption cross-sections of $\mathrm{AlF}$ and $\mathrm{AlCl}$ calculated at the TD-B3LYP//6-311+g(2d,p) level of theory (Frisch et al. 2016). Photolysis is possible in the portions of the absorption curves shown with solid lines. 